\title{
Dobrushin-Kotecký-Shlosman theorem for polygonal Markov fields in the plane
}

\author{
Tomasz Schreiber, ${ }^{* \dagger}$ \\ Faculty of Mathematics \& Computer Science, \\ Nicolaus Copernicus University, \\ Toruń, Poland.
}

\begin{abstract}
We consider the so-called length-interacting Arak-Surgailis polygonal Markov fields with $V$-shaped nodes - a continuum and isometry invariant process in the plane sharing a number of properties with the two-dimensional Ising model. For these polygonal fields we establish a low-temperature phase separation theorem in the spirit of the Dobrushin-Kotecky-Shlosman theory, with the corresponding Wulff shape deteremined to be a disk due to the rotation invariant nature of the considered model. As an important tool replacing the classical cluster expansion techniques and very well suited for our geometric setting we use a graphical construction built on contour birth and death process, following the ideas of Férnandez, Ferrari and Garcia.
\end{abstract}

Keywords: phase separation, DKS theorem, Wulff shape, Arak-Surgailis polygonal Markov fields

${ }^{*}$ Research supported by the Foundation for Polish Science (FNP), by the Polish Minister of Scientific Research and Information Technology grant 1 P03A 01828 (2005-2007) and from the EC 6th Framework Programme Priority 2 Information Society Technology Network of Excellence MUSCLE (Multimedia Understanding through Semantics, Computation and Learning; FP6-507752). A part of this research was completed while staying at the Centrum voor Wiskunde en Informatica (CWI), Amsterdam, The Netherlands

${ }^{\dagger}$ Mailing address: Tomasz Schreiber, Faculty of Mathematics \& Computer Science, Nicolaus Copernicus University, ul. Chopina 12 / 18, 87-100 Toruń, Poland; tel.: $(++48)(+56) 6113442$, fax: $(++48)(+56)$ 6228979; e-mail: tomeks at mat.uni.torun.pl 


\section{Introduction and main results}

An example of a planar polygonal Markov field, referred to as the Arak process throughout this paper, was first introduced by Arak [1]. Further developments are due to Arak \& Surgailis [2],[3], Surgailis [16], Arak, Clifford \& Surgailis [4]. In this paper we focus our attention on polygonal Markov fields with V-shaped nodes, which are a particular class of ensembles of self-avoiding polygonal loops (contours) in the plane, interacting only by the requirement of disjointness. Not unexpectedly, these objects share a number of properties of the two-dimensional Ising model, including the presence of spontaneous magnetisation and absence of infinite contour nesting in low temperature region, see Nicholls [13] and Schreiber [15]. An important property of the Arak process and its length-interacting Gibbsian modifications is their isometry invariance. One might be tempted to view these purely continuum polygonal fields as a kind of continuum version of the Ising model. For low temperatures this opinion seems to be well founded. There is a number of relevant differences though in the much less understood high temperature region. In sharp contrast to the Ising model it is not clear how to define the infinite temperature non-interacting field, since some spatial correlation is always present due to the imposed polygonal nature of the contours. Therefore as the reference field for length-interacting Gibbsian modifications we choose the original Arak process, enjoying a number of striking properties including consistency, exact solubility and admitting the so-called dynamic representation in terms of equilibrium evolution of one-dimensional particle systems tracing the polygonal boundaries of the process in two-dimensional space-time, see Arak \& Surgailis [2] and the Appendix below for details.

The purpose of this paper is to show that, in analogy with the Ising model, the phase separation phenomenon is present for length-interacting polygonal Markov fields and it is gouverned by the Wulff construction, see Bodineau, Ioffe \& Velenik [6] for an extensive reference. We establish our main Theorem 1.2 in the DKS set-up, as introduced by Dobrushin, Kotecký \& Shlosman in their seminal monograph [7], and we only work at low enough temperatures. As a crucial tool replacing cluster expansion techniques and very well suited for our geometric setting we use a graphical construction built on contour birth and death process, as introduced by Férnandez, Ferrari \& Garcia [9],[10],[11], see Subsection 1.2. We took advantage of the particular properties of polygonal fields in order to characterise the model-specific surface tension, defined in Subsection 1.3, in terms of 
hitting probabilities of appropriate planar random walks in random environment provided by the graphical construction. Even though we were only able to establish relatively weak results for the quality of approximation of the surface tension by its finite volume versions, we used the isometry invariance of the model to circumvent this problem. A particular feature of our approach is that rather than imposing periodic [as in Dobrushin, Kotecký \& Shlosman [7]] or fixed sign boundary conditions [as Ioffe \& Schonmann [12]], we work directly in the thermodynamic limit conditioned on the event that no large contours hit the boundary of the region. This allows us to avoid technical difficulties which would arise if we had to control our surface tension estimates in close vicinity of the boundary. Finally, the micro-canonical constraint considered in this paper requires that the excess of total magnetisation be larger or equal rather than just equal to a given positive threshold value - this avoids a number of technical complications which would otherwise arise due to the continuum nature of our setting and allows us to work with weaker versions of moderate deviation estimates and to rely upon general local central limit (LCL) results available in the literature rather than establishing an LCL theorem in its full strength specialised for our model.

In analogy to the original DKS approach, the crucial ingredients of the proof of our main Theorem 1.2 are

- the coarse graining estimates of Section 6, based on skeleton techniques slightly modified and specialised for our particular setting. The graphical construction of Subsection 1.2 is used as a crucial tool replacing cluster expansion techniques,

- moderate deviation estimates for cut-off ensembles, stated in Section 3 and established by the classical method of studying the restricted phase modified by actions of finely-tuned external magnetic fields, see e.g. Section 2 of Ioffe \& Schonmann [12]. The graphical construction of Subsection 1.2 admits an extension for these modified fields.

We believe the techniques developed in the present paper should in principle be applicable to general continuum models exhibiting isometry invariance and admitting polymer representation.

The remaining part of the introductory section is organised as follows. Below, in Subsection 1.1 we give a formal construction of the polygonal Markov fields. The next Subsection 
1.2 is devoted to the graphical construction. The surface tension specific for our model is defined in Subsection 1.3. Finally, our main results are formulated in Subsection 1.4.

Throughout the paper we make extensive use of the ' $O, \Omega, \Theta^{\prime}$ notation, with $O(X)$ and $\Omega(X)$ standing respectively for quantities bounded in their absolute value above and below by a constant times $X$, and with $\Theta(X)=O(X) \cap \Omega(X)$. Moreover, we use $c, C, C_{1}, C_{2}$ etc. to denote generic constants which can change their values from one statement to another.

\subsection{Length-interacting polygonal Markov fields}

The formal construction of the basic Arak process with empty boundary conditions in a bounded open set $D \subseteq \mathbb{R}^{2}$ goes as briefly discussed below [we refer the reader to [2] and [4] for further details]. In the sequel we assume that the boundary $\partial D$ is piecewise smooth. We define the family $\Gamma_{D}$ of admissible polygonal configurations on $D$ by taking all the planar graphs $\gamma$ in $D$ such that

(P1) $\gamma \cap \partial D=\emptyset$

(P2) all the vertices of $\gamma$ are of degree 2,

(P3) the edges of $\gamma$ do not intersect,

(P4) no two edges of $\gamma$ are co-linear.

In other words, $\gamma$ consists of a finite number of disjoint polygons fully contained in $D$ and possibly nested. Further, for a finite collection $(l)=\left(l_{i}\right)_{i=1}^{n}$ of straight lines intersecting $D$ we denote by $\Gamma_{D}(l)$ the family of admissible configurations $\gamma$ with the additional properties that $\gamma \subseteq \bigcup_{i=1}^{n} l_{i}$ and $\gamma \cap l_{i}$ is a single interval of a strictly positive length for each $l_{i}, i=1, \ldots, n$, possibly with some isolated points added. Let $\Lambda_{D}$ be the restriction to $D$ of a homogeneous Poisson line process $\Lambda$ with intensity measure given by the standard isometry-invariant Lebesgue measure $\mu$ on the space of straight lines in $\mathbb{R}^{2}$. One possible construction of $\mu$ goes by identifying a straight line $l$ with the pair $(\phi, \rho) \in[0, \pi) \times \mathbb{R}$, where $(\rho \sin (\phi), \rho \cos (\phi))$ is the vector orthogonal to $l$ and joining it to the origin, and then by endowing the parameter space $[0, \pi) \times \mathbb{R}$ with the usual Lebesgue measure. With the above notation, the basic polygonal Arak process $\mathcal{A}_{D}$ on $D$ arises as the Gibbsian modification of the process induced on $\Gamma_{D}$ by $\Lambda_{D}$, with the Hamiltonian given by the double total edge 
length, that is to say

$$
\mathbb{P}\left(\mathcal{A}_{D} \in G\right)=\frac{\mathbb{E} \sum_{\gamma \in \Gamma_{D}\left(\Lambda_{D}\right) \cap G} \exp (-2 \text { length }(\gamma))}{\mathbb{E} \sum_{\gamma \in \Gamma_{D}\left(\Lambda_{D}\right)} \exp (-2 \text { length }(\gamma))}
$$

for all $G \subseteq \Gamma_{D}$ Borel measurable, say with respect to the usual Hausdorff distance topology, and where $\Gamma_{D}\left(\Lambda_{D}\right)$ denotes $\Gamma_{D}(l)$ as defined above with $l$ set to be the collection of all straight lines of $\Lambda_{D}$. Note that by the total edge length length $(\gamma)$ of a polygonal configuration $\gamma$ we mean here and below the sum of lengths of all constituent polygons. The expectations in (1.1) are taken with respect to the randomness of $\Lambda_{D}$. It should be mentioned at this point that in the literature on consistent polygonal fields one usually considers free rather than empty boundary conditions, see [2] and the Appendix below, yet the empty boundary object is better suited for the graphical construction below and for our further purposes.

For a positive inverse temperature $\beta>0$ we consider the length-interacting Arak process $\mathcal{A}_{D}^{[\beta]}$ in $D$ determined in distribution by

$$
\frac{d \mathcal{L}\left(\mathcal{A}_{D}^{[\beta]}\right)}{d \mathcal{L}\left(\mathcal{A}_{D}\right)}[\gamma]:=\frac{\exp (-\beta \text { length }(\gamma))}{\mathbb{E} \exp \left(-\beta \text { length }\left(\mathcal{A}_{D}\right)\right)},
$$

with $\mathcal{L}(\cdot)$ standing for the law of the argument random object. As shown in Theorem 3 and Corollary 4 of [15] for $\beta$ large enough the polygonal fields $\mathcal{A}_{D}^{[\beta]}, D \subseteq \mathbb{R}^{2}$, admit a unique whole plane thermodynamic limit without infinite contours, denoted in the sequel by $\mathcal{A}^{[\beta]}$, see also below for its construction. The field $\mathcal{A}^{[\beta]}$ is isometry invariant. The thermodynamic limit $\mathcal{A}^{[0]}$ can also be shown to exist for $\beta=0$, in the sequel it is denoted by $\mathcal{A}$ and its construction is given in the Appendix.

It is known that for the inverse temperature $\beta$ sufficiently large (in particular, for all $\beta$ within the validity region of the graphical construction below) the thermodynamic limit $\mathcal{A}^{[\beta]}$ exhibits only finite contour nesting, see Nicholls [13] and the discussion following Corollary 4 in Schreiber [15]. Whence, the contour ensemble $\mathcal{A}^{[\beta]}$ partitions the plane into a unique infinite connected component (the ocean) and a countable number of finitely nested bounded regions (islands). We colour black and white the polygonal regions of this partition by declaring the infinite ocean white and by requiring that the collection of interfaces between black and white regions coincide with the collection of contours $\mathcal{A}^{[\beta]}$, which uniquely determines the colouring. Denote the resulting union of black regions by $\operatorname{black}\left[\mathcal{A}^{[\beta]}\right]$ and the union of white regions by white $\left[\mathcal{A}^{[\beta]}\right]$. For a bounded region $U \subseteq \mathbb{R}^{2}$ let $\mathbb{M}_{U}\left(\mathcal{A}^{[\beta]}\right)$ be the magnetisation in $U$ determined by the coloured contour ensemble $\mathcal{A}^{[\beta]}$ 
under the assignment black $\mapsto+$, white $\mapsto-$. In other words, $\mathbb{M}_{U}\left(\mathcal{A}^{[\beta]}\right)$ is the total area of the black-coloured regions in $U$ minus the total area of white-coloured regions in $U$ :

$$
\mathbb{M}_{U}\left(\mathcal{A}^{[\beta]}\right):=\text { Area }\left(\operatorname{black}\left[\mathcal{A}^{[\beta]}\right] \cap U\right)-\text { Area }\left(\text { white }\left[\mathcal{A}^{[\beta]}\right] \cap U\right)
$$

For $L>0$ we shall abbreviate $\mathbb{M}_{\mathbb{B}_{2}(L)}\left(\mathcal{A}^{[\beta]}\right)$ to $\mathbb{M}_{L}[\beta]$, where $\mathbb{B}_{2}(L)$ stands for the disk of radius $L$ centred at 0 . The isometry invariance of the infinite-volume field $\mathcal{A}^{[\beta]}$ implies that

$$
\mathbb{E} \mathbb{M}_{U}\left(\mathcal{A}^{[\beta]}\right)=\operatorname{Area}(U) \mathbb{M}[\beta], \quad \mathbb{M}[\beta] \in(-1,0),
$$

where $|\mathbb{M}[\beta]|$ is further referred to as the specific spontaneous magnetisation at inverse temperature $\beta$. The 'black[.]' and 'M. $(\cdot)$ ' notation will be also used for $\mathcal{A}^{[\beta]}$ replaced by a number of other polygonal fields enjoying the property that the corresponding contour ensemble determines a unique unbounded region, to be coloured white.

\subsection{Graphical construction}

\subsubsection{Basic graphical construction}

As argued in Schreiber [15], the polygonal field $\mathcal{A}_{D}^{[\beta]}$ admits a natural representation in terms of a graphical construction in the spirit of Fernández, Ferrari \& Garcia [9],[10],[11], which will be a crucial tool in our argument in the sequel, as replacing cluster expansion techniques. Below, we provide a description of this construction borrowed from [15]. Consider the space $\mathcal{C}_{D}$ consisting of all closed polygonal contours in $D$ which do not touch the boundary $\partial D$. For a given finite configuration $(l):=\left(l_{1}, \ldots, l_{n}\right)$ of straight lines intersecting $D$ denote by $\mathcal{C}_{D}(l)$ the family of those polygonal contours in $\mathcal{C}_{D}$ which belong to $\Gamma_{D}(l)$. We define the so-called free contour measure $\Theta_{D}$ on $\mathcal{C}_{D}$ by putting for $C \subseteq \mathcal{C}_{D}$ measurable, say with respect to the Borel $\sigma$-field generated by the Hausdorff distance topology,

$$
\Theta_{D}(C)=\int_{\operatorname{Fin}(L[D])} \sum_{\theta \in C \cap \mathcal{C}_{D}(l)} \exp (-2 \text { length }(\theta)) d \mu^{*}((l))
$$

with $\operatorname{Fin}(L[D])$ standing for the for the family of finite line configurations intersecting $D$ and where $\mu^{*}$ is the measure on $\operatorname{Fin}(L[D])$ given by $d \mu^{*}\left(\left(l_{1}, \ldots, l_{n}\right)\right):=\prod_{i=1}^{n} d \mu\left(l_{i}\right)$ with $\mu$ defined in the discussion preceding (1.1).

For $\beta>0$ we consider the exponential modification $\Theta_{D}^{[\beta]}$ of the free measure $\Theta_{D}$,

$$
\Theta_{D}^{[\beta]}(d \theta):=\exp (-\beta \text { length }(\theta)) \Theta_{D}(d \theta)
$$


Observe that for all bounded open sets $D$ with piecewise smooth boundary the free contour measures $\Theta_{D}$ as defined in (1.4) arise as the respective restrictions to $\mathcal{C}_{D}$ of the same measure $\Theta$ on $\mathcal{C}:=\bigcup_{n=1}^{\infty} \mathcal{C}_{(-n, n)^{2}}$, in the sequel referred to as the infinite volume free contour measure. Indeed, this follows easily by the observation that $\Theta_{D_{1}}$ restricted to $\mathcal{C}_{D_{2}}$ coincides with $\Theta_{D_{2}}$ for $D_{2} \subseteq D_{1}$. In the same way we construct the infinite-volume Gibbs-modified measures $\Theta^{[\beta]}$. The following result, which is Lemma 1 of [15] (note that the first result in this spirit is due to Nicholls [13], see Lemma in the Appendix ibidem) will be crucial for our further purposes.

Lemma 1.1 For $\beta \geq 2$ we have

$$
\Theta^{[\beta]}(\{\theta \mid d x \cap \operatorname{Vertices}(\theta) \neq \emptyset, \text { length }(\theta)>R\}) \leq 8 \pi \exp (-[\beta-2] R) d x
$$

where the event $\{d x \cap \operatorname{Vertices}(\theta) \neq \emptyset\}$ is to be understood that a vertex of $\theta$ falls into $d x$. Moreover, there exists a constant $\varepsilon>0$ such that, for $\beta \geq 2$,

$$
\Theta^{[\beta]}(\{\theta \mid \mathbf{0} \in \operatorname{Int} \theta, \text { length }(\theta)>R\}) \leq \exp (-[\beta-2+\varepsilon] R+o(R)),
$$

with Int $\theta$ standing for the region enclosed by $\theta$ (recall that $\theta \in \mathcal{C}$ is always a single bounded contour).

Let $\mathcal{P}_{\Theta_{D}^{[\beta]}}$ be the Poisson point process on $\mathcal{C}_{D}$ with intensity measure $\Theta_{D}^{[\beta]}$. It follows then directly by (1.4) and by (1.1) that $\mathcal{A}_{D}^{[\beta]}$ coincides in distribution with the union of contours in $\mathcal{P}_{\Theta_{D}^{[\beta]}}$ conditioned on the event that they are disjoint so that

$$
\mathcal{L}\left(\mathcal{A}_{D}^{[\beta]}\right)=\mathcal{L}\left(\bigcup_{\theta \in \mathcal{P}_{\Theta_{D}^{[\beta]}}} \theta \mid \forall_{\theta, \theta^{\prime} \in \mathcal{P}_{\Theta_{D}^{[\beta]}}} \theta \neq \theta^{\prime} \Rightarrow \theta \cap \theta^{\prime}=\emptyset\right)
$$

where the conditioning makes sense because $\Theta_{D}^{[\beta]}\left(\mathcal{C}_{D}\right)$ is finite as shown in Subsection 2.2 of [15]. In particular, as argued in Subsection 2.2 and Theorem 2 ibidem, the law of $\mathcal{A}_{D}^{[\beta]}$ is invariant and reversible with respect to the following contour birth and death dynamics $\left(\gamma_{s}^{D}\right)$ on $\Gamma_{D}$.

$(\mathbf{C}: \operatorname{birth}[\beta])$ With intensity $\Theta_{D}^{[\beta]}(d \theta) d s$ do

- Choose a new contour $\theta$, 
- If $\theta \cap \gamma_{s}^{D}=\emptyset$, accept $\theta$ and set $\gamma_{s+d s}^{D}:=\gamma_{s}^{D} \cup \theta$,

- Otherwise reject $\theta$ and keep $\gamma_{s+d s}^{D}:=\gamma_{s}^{D}$,

$(\mathbf{C}: \operatorname{death}[\beta])$ With intensity $1 \cdot d s$ for each contour $\theta \in \gamma_{s}^{D}$ remove $\theta$ from $\gamma_{s}^{D}$ setting $\gamma_{s}^{D}:=\gamma_{s}^{D} \backslash \theta$.

Moreover, $\mathcal{L}\left(\mathcal{A}_{D}^{[\beta]}\right)$ is the unique invariant distribution of the above dynamics, see Theorem 2 in [15]. These observations place us within the framework of the general contour birth and death graphical construction as developed by Fernández, Ferrari \& Garcia [9],[10],[11] and as briefly sketched below, see ibidem and Schreiber [15] for further details. Choose $\beta$ large enough, to be specified below. Define $\mathcal{F}(\mathcal{C})$ to be the space of countable and locally finite collections of contours from $\mathcal{C}$, with the local finiteness requirement meaning that at most a finite number of contours can hit a bounded subset of $\mathbb{R}^{2}$. On the time-space $\mathbb{R} \times \mathcal{F}(\mathcal{C})$ we construct the stationary free contour birth and death process $\left(\varrho_{s}\right)_{s \in \mathbb{R}}$ with the birth intensity measure given by $\Theta^{[\beta]}$ and with the death intensity 1 . Note that free means here that every new-born contour is accepted regardless of whether it hits the union of already existing contours or not, moreover we admit negative time here, letting $s$ range through $\mathbb{R}$ rather than just $\mathbb{R}_{+}$. Observe also that we need the birth measure $\Theta^{[\beta]}$ to be finite on the sets $\{\theta \in \mathcal{C} \mid \theta \cap A \neq \emptyset\}$ for all bounded Borel $A \subseteq \mathbb{R}^{2}$ in order to have the process $\left(\varrho_{s}\right)_{s \in \mathbb{R}}$ well defined on $\mathbb{R} \times \mathcal{F}(\mathcal{C})$. By Lemma 1.1 this is ensured whenever $\beta \geq 2$. To proceed, for the free process $\left(\varrho_{s}\right)_{s \in \mathbb{R}}$ we perform the following trimming procedure. We place a directed connection from each time-space instance of a contour showing up in $\left(\varrho_{s}\right)_{s \in \mathbb{R}}$ and denoted by $\theta \times\left[s_{0}, s_{1}\right)$, with $\theta$ standing for the contour and $\left[s_{0}, s_{1}\right)$ for its lifespan, to all time-space contour instances $\theta^{\prime} \times\left[s_{0}^{\prime}, s_{1}^{\prime}\right)$ with $\theta^{\prime} \cap \theta \neq \emptyset, s_{0}^{\prime} \leq s_{0}$ and $s_{1}^{\prime}>s_{0}$. In other words, we connect $\theta \times\left[s_{0}, s_{1}\right)$ to those contour instances which may have affected the acceptance status of $\theta \times\left[s_{0}, s_{1}\right)$ in the constrained contour birth and death dynamics (C) as discussed above. These directed connections give rise to directed ancestor chains of time-space contour instances, following [11] the union of all ancestor chains stemming from a given contour instance $\theta^{*}=\theta \times\left[s_{0}, s_{1}\right)$, including the instance itself, is referred to as its clan of ancestors and is denoted by $\operatorname{An}\left(\theta^{*}\right)$. More generally, for a bounded region $U$ in the plane we write $\operatorname{An}_{s}(U)$ for the union of ancestor clans of all contour instances $\theta \times\left[s_{0}, s_{1}\right)$ with $\theta \cap U \neq \emptyset$ and $s \in\left[s_{0}, s_{1}\right)$. Lemma 1.1 allows us to apply the technique of domination by sub-critical branching processes, developed in [9],[10],[11], in order to 
conclude that there exists $\beta_{g}$ such that for each $\beta>\beta_{g}$ there exists $c:=c(\beta)>0$ such that

$$
\mathbb{P}\left(\operatorname{diam} \operatorname{An}_{s}\left(\mathbb{B}_{2}(x, 1)\right)>R\right) \leq \exp (-c R), s \in \mathbb{R}, x \in \mathbb{R}^{2},
$$

with $\mathbb{B}_{2}(x, 1)$ standing for the radius 1 ball in $\mathbb{R}^{2}$ centred at $x$. In the sequel we shall always assume that $\beta>\beta_{g}$, that is to say that $\beta$ is in the validity region of the graphical construction. We see that for $\beta>\beta_{g}$ all the ancestor clans are a.s. finite and we can uniquely determine the acceptance status of all their members: contour instances with no ancestors are a.s. accepted, which automatically and uniquely determines the acceptance status of all the remaining members of the clan by recursive application of the inter-contour exclusion rule. In this case, discarding the unaccepted contour instances leaves us with a time-space representation of a stationary evolution $\left(\gamma_{s}\right)_{s \in \mathbb{R}}$ on $\mathcal{F}(\mathcal{C})$, which is easily checked to evolve according to the whole-plane version of the dynamics (C) above. In Section 4 and Theorem 4 of [15] we argue that for all $s \in \mathbb{R}$ the polygonal field $\gamma_{s}$ coincides in distribution with the thermodynamic limit (see Section 3 ibidem) for $\mathcal{A}^{[\beta]}$ without infinite contours, which is unique (see Corollary 4 ibidem). It should be observed that for each $s \in \mathbb{R}$ the free field $\varrho_{s}$ coincides in distribution with the Poisson contour process $\mathcal{P}_{\Theta[\beta]}$. Since almost surely we have $\gamma_{s} \subseteq \varrho_{s}$, we get the stochastic domination of the contour ensemble $\mathcal{A}^{[\beta]}$ by $\mathcal{P}_{\Theta[\beta]}$.

We also consider finite-volume versions of the above graphical construction, replacing the infinite-volume birth intensity measure $\Theta^{[\beta]}$ with its finite-volume counterparts $\Theta_{D}^{[\beta]}$ for bounded and open $D$ with piecewise smooth boundary. Clearly, the graphical construction yields then a version of the finite-volume contour birth and death evolution $(\mathbf{C})$. For each $D$ denote by $\left(\gamma_{s}^{D}\right)_{s \in \mathbb{R}}$ the resulting finite-volume stationary process on the space $\mathcal{F}\left(\mathcal{C}_{D}\right)$ of finite contour configurations in $D$ and write $\left(\varrho_{s}^{D}\right)_{s \in \mathbb{R}}$ for the corresponding free process. It follows by Theorem 2 in [15] that $\gamma_{s}^{D}$ coincides in distribution with $\mathcal{A}_{D}^{[\beta]}$ for each $s \in \mathbb{R}$. Likewise, $\varrho_{s}^{D}$ coincides in distribution with $\mathcal{P}_{\Theta_{D}^{[\beta]}}$.

By representing the measures $\Theta_{D}^{[\beta]}$ as the corresponding restrictions of $\Theta^{[\beta]}$ we obtain a natural coupling of all the processes $\gamma_{s}^{D}, \varrho_{s}^{D}, \gamma_{s}$ and $\varrho_{s}$ on a common probability space. We shall also consider $\mathcal{A}_{D}^{[\beta]}$ coupled on the same probability space by putting $\mathcal{A}_{D}^{[\beta]}=\gamma_{0}^{D}$. Likewise, we put $\mathcal{A}^{[\beta]}=\gamma_{0}, \mathcal{P}_{\Theta[\beta]}=\varrho_{0}, \mathcal{P}_{\Theta_{D}^{[\beta]}}=\varrho_{0}^{D}$. This coupling, referred to as the canonical coupling in the sequel, will be assumed without a further mention throughout this paper. 
A simple yet useful application of this coupling is that

$$
\left|\operatorname{Area}(D) \mathbb{M}[\beta]-\mathbb{E} \mathbb{M}_{D}\left(\mathcal{A}_{D}^{[\beta]}\right)\right|=O\left(\operatorname{Area}\left(\partial D \oplus \mathbb{B}_{2}(1)\right)\right)
$$

with $\oplus$ standing for the usual Minkowski addition [i.e. $X \oplus Y:=\{x+y \mid x \in X, y \in Y\}$ ]. Indeed, this is immediately seen by observing that, by Lemma 1.1 and in view of (1.7) stating the exponential tail decay for ancestor clan diameters, under the canonical coupling of $\mathcal{A}_{D}^{[\beta]}$ and $\mathcal{A}^{[\beta]}$, the probability that a given point $x \in D$ is assigned different colours by these ensembles decays as $\exp (-\Omega(\operatorname{dist}(x, \partial D)))$. Integrating over $D$ and using (1.3) we obtain the required relation.

\subsubsection{Modifications of the basic graphical construction}

Below we discuss a number of modifications of the graphical construction, which will be of use for our further purposes. Apart from the area-interacting modifications all the remaining ones can be defined on the probability space of the basic construction, thus extending the canonical coupling.

Imposing forbidden regions A particular property of the graphical construction which will be crucial for our further purposes is that it admits, on the same probability space, conditional versions on the events of the type no contour of the polygonal field hits [intersects] a given region $U$. Indeed, let $U$ be a bounded subset of the plane $\mathbb{R}^{2}$. Then, adding the rule that all new-born contours hitting $U$ [intersections of $U$ only with the interior of a contour are not taken into account] be immediately discarded, to the trimming procedure constructing $\left(\gamma_{s}\right)$ out of $\left(\varrho_{s}\right)$ or, equivalently, to the dynamics $(\mathbf{C})$, we obtain a stationary and reversible process $\left(\gamma_{s: U}\right)$ easily seen to enjoy the property that the distribution of $\gamma_{s: U}$ for each fixed $s$ coincides with the law of $\mathcal{A}^{[\beta]}$ conditioned on the event that $\mathcal{A}^{[\beta]} \cap U=\emptyset$. Put $\mathcal{A}_{\mathbb{R}^{2}: U}^{[\beta]}:=\gamma_{0: U}$. Likewise, we define the conditioned version $\left(\gamma_{s: U}^{D}\right)$ of the finite volume process $\left(\gamma_{s}^{D}\right)$ for which the distribution of $\gamma_{s: U}^{D}$ coincides for each $s \in \mathbb{R}$ with the law of $\mathcal{A}_{D}^{[\beta]}$ conditioned on $\left\{\mathcal{A}_{D}^{[\beta]} \cap U=\emptyset\right\}$. We put $\mathcal{A}_{D: U}^{[\beta]}:=\gamma_{0: U}^{D}$. In full analogy with the similar discussion above, the conditioned field $\mathcal{A}_{\mathbb{R}^{2}: U}^{[\beta]}$ is stochastically bounded by the Poisson contour process $\mathcal{P}_{\Theta[\beta]: U}:=\left\{\theta \in \mathcal{P}_{\Theta[\beta]} \mid \theta \cap U=\emptyset\right\}$. Likewise, $\mathcal{A}_{D: U}^{[\beta]}$ is stochastically bounded by $\mathcal{P}_{\Theta_{D}^{[\beta]}: U}:=\left\{\theta \in \mathcal{P}_{\Theta_{D}^{[\beta]}} \mid \theta \cap U=\emptyset\right\}$.

Cut-off ensembles An important family of processes we embed into the original graphical construction are the cut-off ensembles for $\mathcal{A}^{[\beta]}$. They are defined as follows. For a 
positive cut-off threshold $\alpha$ and a bounded region $V \subseteq \mathbb{R}^{2}$ we consider the measure $\Theta^{[\beta] ; \alpha, V}$ which is the restriction of $\Theta^{[\beta]}$ to the family of polygonal contours which either do not hit $V$, or if they do hit $V$ then their diameter does not exceed $\alpha$. In this context, it is convenient to say that a contour $\gamma$ is $\alpha$-large iff $\operatorname{diam}(\gamma)>\alpha$ and that it is $\alpha$-small otherwise. Using $\Theta^{[\beta] ; \alpha, V}$ instead of $\Theta^{[\beta]}$ for the contour birth intensity in the graphical construction we obtain $\alpha$-cut-off version $\left(\gamma_{s}^{\mathbb{R}^{2} ; \alpha, V}\right)_{s \in \mathbb{R}}$ of the process $\left(\gamma_{s}\right)_{s \in \mathbb{R}}$ (equivalently, we can simply reject all $\alpha$-large contours hitting $V$ upon their birth in the original graphical construction, which naturally extends the canonical coupling). Put $\mathcal{A}^{[\beta] ; \alpha, V}:=\gamma_{0}^{\mathbb{R}^{2} ; \alpha, V}$. It is easily seen that the $\alpha$-cut-off polygonal field $\mathcal{A}^{[\beta] ; \alpha, V}:=\gamma_{0}^{\mathbb{R}^{2} ; \alpha, V}$ coincides in law with $\mathcal{A}^{[\beta]}$ conditioned on the event that no contour hitting $V$ has its diameter larger than $\alpha$. Likewise, we consider with obvious definition the finite volume cut-off processes $\left(\gamma_{s}^{D ; \alpha, V}\right)_{s \in \mathbb{R}}$ for open and bounded $D$ with piecewise smooth boundary. Clearly, the finite volume $\alpha$-cut-off polygonal field $\mathcal{A}_{D}^{[\beta] ; \alpha, V}:=\gamma_{0}^{D ; \alpha, V}$ arises as $\mathcal{A}_{D}^{[\beta]}$ conditioned on the event that no contour hitting $V$ is $\alpha$-large. In analogy with the similar discussion above, the cut-off field $\mathcal{A}^{[\beta] ; \alpha, V}$ is stochastically dominated by $\mathcal{P}_{\Theta[\beta] ; \alpha, V}$ and $\mathcal{A}_{D}^{[\beta] ; \alpha, V}$ is stochastically dominated by $\mathcal{P}_{\Theta_{D}^{[\beta] ; \alpha, V}}$.

Clearly, we can combine the cut-off operation with imposing a forbidden region which leads to processes $\gamma_{s: U}^{\mathbb{R}^{2} ; \alpha, V}, \gamma_{s: U}^{D ; \alpha, V}, \mathcal{A}_{\mathbb{R}^{2}: U}^{[\beta] \alpha, V}$ and $\mathcal{A}_{D: U}^{[\beta] ;, V}$ with obvious definitions, stochastically dominated by $\mathcal{P}_{\Theta[\beta] ; \alpha, V: U}$ and $\mathcal{P}_{\Theta_{D}^{[\beta] ; \alpha, V}: U}$ respectively. The canonical coupling is extended in the obvious way.

Area-interacting fields The final modification considered involves introducing an areaorder term to the Hamiltonian of (1.2). To this end, for a bounded region $W \subseteq \mathbb{R}^{2}$ and $h \in \mathbb{R}$ we consider the polygonal field $\mathcal{A}_{W}^{[\beta, h]}$ on $W$, given in distribution by

$$
\frac{d \mathcal{L}\left(\mathcal{A}_{W}^{[\beta, h]}\right)}{d \mathcal{L}\left(\mathcal{A}^{[\beta]} \cap W\right)}[\gamma]=\frac{\exp \left(h \mathbb{M}_{W}(\gamma)\right)}{\mathbb{E} \exp \left(h \mathbb{M}_{W}\left(\mathcal{A}^{[\beta]}\right)\right)} .
$$

Note that, unlike $\mathcal{A}_{D}^{[\beta]}$, the field $\mathcal{A}_{W}^{[\beta, h]}$ is defined as a Gibbsian modification of the thermodynamic limit $\mathcal{A}^{[\beta]}$ restricted to $W$ rather than as a Gibbsian modification of the finite volume field $\mathcal{A}_{W}$. In particular, the laws of the fields $\mathcal{A}_{W}^{[\beta, 0]}$ and $\mathcal{A}_{W}^{[\beta]}$ do not coincide; in fact $\mathcal{A}_{W}^{[\beta, 0]}$ coincides in distribution with $\mathcal{A}^{[\beta]} \cap W$. We will mainly use the area-interacting modification combined with the cut-off operation. The field $\mathcal{A}_{W}^{[\beta, h] ; \alpha, V}$ is given in law by

$$
\frac{d \mathcal{L}\left(\mathcal{A}_{W}^{[\beta, h] ; \alpha, V}\right)}{d \mathcal{L}\left(\mathcal{A}^{[\beta] ; \alpha, V} \cap W\right)}[\gamma]=\frac{\exp \left(h \mathbb{M}_{W}(\gamma)\right)}{\mathbb{E} \exp \left(h \mathbb{M}_{W}\left(\mathcal{A}^{[\beta] ; \alpha, V}\right)\right)} .
$$


To proceed with the graphical construction we assume that

$$
|h| \leq \frac{\beta}{\pi \alpha}
$$

and observe that adding a single $\alpha$-small contour $\theta$ to a contour configuration $\gamma, \gamma \cap \theta=$ $\emptyset$, can change the magnetisation $\mathbb{M}_{W}(\gamma)$ by at most $\pi \operatorname{length}(\theta)^{2} / 2$ whence the value of $h \mathbb{M}_{W}(\gamma)$ can change by at most $\beta$ length $(\theta) / 2$. With $\gamma$ standing for the current contour configuration, we modify the original graphical construction by

- constructing the free birth and death process $\hat{\varrho}_{s}, s \in \mathbb{R}$, with birth intensity measure $\Theta^{[\beta / 2] ; \alpha, V}$ and death intensity 1 ,

- at the trimming stage, by accepting a time-space contour instance $\theta \times\left[s_{0}, s_{1}\right)$

- with probability 0 if $\theta$ hits $\theta^{\prime}$ for some previously accepted contour instance $\theta^{\prime} \times\left[s_{0}^{\prime}, s_{1}^{\prime}\right)$ alive at time $s_{0}$,

- with probability $\exp \left(-\frac{\beta}{2} \operatorname{length}(\theta)+h\left[\mathbb{M}_{W}(\gamma \cup \theta)-\mathbb{M}_{W}(\gamma)\right]\right)$ otherwise.

Observe that the last probability falls into $(0,1]$ because of $(1.9)$. Denote the resulting trimmed process by $\hat{\gamma}_{s}$. The validity of this construction requires a justification. In fact, we have to redefine here the notion of an ancestor clan. We set a directed connection from a contour instance $\theta^{*}=\theta \times\left[s_{0}, s_{1}\right)$ to all contour instances $\theta^{\prime *}=\theta^{\prime} \times\left[s_{0}^{\prime}, s_{1}^{\prime}\right)$ such that Int $\theta \cap \operatorname{Int} \theta^{\prime} \neq \emptyset$ (which is weaker than the condition $\theta \cap \theta^{\prime} \neq \emptyset$ of the original definition) and $s_{0}^{\prime} \leq s_{0}, s_{1}^{\prime}>s_{0}$. Clearly, these are all contour instances which may affect the acceptance status of $\theta^{*}$. The union of all the directed chains stemming from $\theta^{*}$ is called the ancestor clan of $\theta^{*}$ and denoted by $\hat{\mathrm{An}}\left(\theta^{*}\right)$. Likewise, for $s \in \mathbb{R}$ and $W \subseteq \mathbb{R}^{2}$ we write $\hat{\mathrm{An}_{s}}(U)$ for the union of all the ancestor chains of contour instances $\theta \times\left[s_{0}, s_{1}\right)$ alive at time $s$ (i.e. $s_{0} \leq s<s_{1}$ ) and such that $\operatorname{Int} \theta \cap U \neq \emptyset$. In full analogy with (1.7), Lemma 1.1 guarantees that for $\beta$ large enough (larger than some $\hat{\beta}_{g}$ ) we have

$$
\mathbb{P}\left(\operatorname{diam} \hat{A n}_{s}\left(\mathbb{B}_{2}(x, 1)\right)>R\right) \leq \exp (-c R), s \in \mathbb{R}, x \in \mathbb{R}^{2},
$$

for some $c=c(\beta)>0$. Clearly, this implies that the ancestor clans are a.s. finite, thus ensuring the validity of the construction. In the sequel we shall always assume that $\beta>\hat{\beta}_{g}$ so that (1.10) holds. It follows by the general theory developed by Férnandez, Ferrari \& Garcia [9],[10],[11], that so constructed $\hat{\gamma}_{s}$ for each fixed $s$ coincides in law with $\mathcal{A}_{W}^{[\beta, h] ; \alpha, V}$. 
Moreover, it is easily seen that, for each $s \in \mathbb{R}, \hat{\varrho}_{s}$ coincides in law with the Poisson contour process $\mathcal{P}_{\Theta^{[\beta / 2] ; \alpha, V} \cap W} \cap$. Consequently, the almost sure inclusion $\hat{\gamma}_{s} \subseteq \hat{\varrho}_{s}$ yields the stochastic domination of $\mathcal{A}_{W}^{[\beta, h] ; \alpha, V}$ by $\mathcal{P}_{\Theta[\beta / 2] ; \alpha, V} \cap W$.

Clearly, the above construction can be easily extended to take into account forbidden regions. For bounded measurable $U \subseteq \mathbb{R}^{2}$ denote by $\mathcal{A}_{W: U}^{[\beta, h] ; \alpha, V}$ the polygonal fields arising by conditioning $\mathcal{A}_{W}^{[\beta, h] ; \alpha, V}$ on none of its contours hitting $U$. It is easily seen that $\mathcal{A}_{W: U}^{[\beta, h] ;, V}$ can be represented by the graphical construction of this paragraph, with the additional rule that all contours hitting $U$ be immediately discarded. In analogy with a similar observation made above for $\mathcal{A}_{W}^{[\beta] ; \alpha, V}$, also here it should be noted that $\mathcal{A}_{W: U}^{[\beta, 0] ; \alpha, V}$ coincides in law with $\mathcal{A}_{\mathbb{R}^{2}: U}^{[\beta] ;, V} \cap W$ rather than with $\mathcal{A}_{W: U}^{[\beta] ;, V}$.

Moreover, in full analogy with the argument above, we see that $\mathcal{A}_{W: U}^{[\beta] ;, V}$ is stochastically dominated in the sense of inclusion by the Poisson contour process $\mathcal{P}_{\Theta^{[\beta / 2] ; \alpha, V}: U} \cap W$.

Note that the above construction provides a natural coupling for area-interacting fields with cut-off and (possibly) forbidden regions imposed, under the constraint (1.9). To distinguish it from the canonical coupling available for fields with no area interaction as discussed above, we shall call this coupling the canonical coupling for area-interacting fields.

\subsection{Surface tension}

The purpose of this section is to define the surface tension functional specific for our model. To this end, for a given bounded and convex domain $D \subseteq \mathbb{R}^{2}$ and $\delta>0$ we consider the family $\mathcal{C}_{D}^{x \leftrightarrow y ; \delta}$ of self-avoiding polygonal paths in $D$ connecting the balls $\mathbb{B}_{2}(x, \delta) \subseteq D$ and $\mathbb{B}_{2}(y, \delta) \subseteq D$, with the additional property that the first and last segments of the paths do not intersect the interiors of the balls $\mathbb{B}_{2}(x, \delta)$ and $\mathbb{B}_{2}(y, \delta)$ respectively, but they do touch their respective boundaries and the intersection points coincide with the initial and final point of the path. In other words, moving along a path in $\mathcal{C}_{D}^{x \leftrightarrow y ; \delta}$ we travel from $\partial \mathbb{B}_{2}(x, \delta)$ to $\partial \mathbb{B}_{2}(y, \delta)$, with the initial segment falling outside $\mathbb{B}_{2}(x, \delta)$ and with the final segment outside $\mathbb{B}_{2}(y, \delta)$, which does not prevent us though from passing through $\mathbb{B}_{2}(x, \delta)$ and $\mathbb{B}_{2}(y, \delta)$ along the remaining segments. Next, we introduce on $\mathcal{C}_{D}^{x \leftrightarrow y ; \delta}$ the free measure $\Theta_{D}^{x \leftrightarrow y ; \delta}$, constructed in full analogy with the definition of the free contour measure as given in (1.4). For a finite configuration $(l)$ of straight lines crossing $D$ write $\mathcal{C}_{D}^{x \leftrightarrow y ; \delta}(l)$ for the collection of those paths in $\mathcal{C}_{D}^{x \leftrightarrow y ; \delta}$ which only contain segments of the lines in $(l)$ and 
exactly one non-zero length segment on each line. For measurable $C \subseteq \mathcal{C}_{D}^{x \leftrightarrow y ; \delta}$ we put

$$
\Theta_{D}^{x \leftrightarrow y ; \delta}(C)=\int_{\operatorname{Fin}(L[D])} \sum_{\theta \in C \cap \mathcal{C}_{D}^{x \leftrightarrow y ; \delta}(l)} \exp (-2 \text { length }(\theta)) d \mu^{*}((l))
$$

with $\operatorname{Fin}(L[D])$ and $\mu^{*}$ as in (1.4). Note that the initial point and the endpoint of the path $\theta$ in the above definition are uniquely determined, respectively as the intersection of the first and last segment of the path with $\partial \mathbb{B}_{2}(x, \delta)$ and $\partial \mathbb{B}_{2}(y, \delta)$. Likewise, we define the $\beta$-tilted measures $\left[\Theta_{D}^{x \leftrightarrow y ; \delta}\right]^{[\beta]}$ by

$$
\left[\Theta_{D}^{x \leftrightarrow y ; \delta}\right]^{[\beta]}(d \theta):=\exp (-\beta \text { length }(\theta)) \Theta_{D}^{x \leftrightarrow y ; \delta}(d \theta)
$$

As observed above for the free contour measures, also the path measures are consistent in that $\Theta_{D}^{x \leftrightarrow y ; \delta}=\left[\Theta_{D^{\prime}}^{x \leftrightarrow y ; \delta}\right]_{\mid D}$ for $D \subseteq D^{\prime}$ and, consequently, we can construct the whole plane free measure $\Theta^{x \leftrightarrow y ; \delta}$ and its tilted version $\left[\Theta^{x \leftrightarrow y ; \delta}\right]^{[\beta]}$, both defined on $\mathcal{C}^{x \leftrightarrow y ; \delta}=\mathcal{C}_{\mathbb{R}^{2}}^{x \leftrightarrow y ; \delta}:=$ $\bigcup_{n=1}^{\infty} \mathcal{C}_{(-n, n)^{2}}^{x \leftrightarrow y ;}$

To proceed, write

$$
T_{(\delta)}^{[\beta]}[x \leftrightarrow y]:=\int_{\mathcal{C}^{x \leftrightarrow y ; \delta}} \mathbb{P}\left(\mathcal{A}^{[\beta]} \cap \theta=\emptyset\right)\left[\Theta^{x \leftrightarrow y ; \delta}\right]^{[\beta]}(d \theta) .
$$

Put $\mathbf{e}_{x}:=(1,0)$, fix some small $\delta>0$ and let

$$
\tau_{\lambda}^{[\beta]}:=-\frac{1}{\lambda} \log T_{(\delta)}^{[\beta]}\left[0 \leftrightarrow \lambda \mathbf{e}_{x}\right] .
$$

The surface tension is defined as the limit

$$
\tau^{[\beta]}:=\lim _{\lambda \rightarrow \infty} \tau_{\lambda}^{[\beta]}=-\lim _{\lambda \rightarrow \infty} \frac{1}{\lambda} \log T_{(\delta)}^{[\beta]}\left[0 \leftrightarrow \lambda \mathbf{e}_{x}\right] .
$$

It is clear that the asymptotic behaviour of $\tau_{\lambda}^{[\beta]}$ as $\lambda \rightarrow \infty$ does not depend on the choice of $\delta$ above - indeed, changing $\delta$ is easily seen to result only in a bounded and uniformly non-zero prefactor before $T_{(\delta)}^{[\beta]}\left[0 \leftrightarrow \lambda \mathbf{e}_{x}\right]$, which is negligible in the logarithmic large $\lambda$ asymptotics. This is why our notation does not take into account the dependency of $\tau_{\lambda}^{[\beta]}$ on $\delta$. The existence, finiteness and strict positivity of the limit in (1.15) for $\beta$ large enough and other properties of the surface tension are discussed in Section 5 below, see Lemma 5.3 there. 


\subsection{Main results}

Our main result below states that, at low enough temperatures, conditioning the whitedominated phase of polygonal Markov field to contain black-coloured regions of total area exceeding its expectation by an area-order quantity results in aggregation of the excess black area and in formation of a macroscopic-size disk-shaped region (Wulff crystal) of black-dominated phase, separated from the outside white phase by a single large contour. Moreover, the probability of such area-order black exceedances exhibits perimeter-order exponential decay.

As shown in Section 2 below, for $\alpha \gg \log L$ with overwhelming probability there are no $\alpha$-large contours of $\mathcal{A}^{[\beta]}$ in $\mathbb{B}_{2}(L)$. Thus, it is natural to consider the regions separated by $\Omega(\log L)$-large contours of $\mathcal{A}^{[\beta]}$ and to assign them, in the obvious way, black or white phase labels. In this language, we show in this paper that the single large contour determining the Wulff shape encloses a disk-shaped portion of black-labeled phase region surrounded by ocean of white-labeled phase.

As already discussed in the introductory section, since our main results are formulated directly under boundary conditions induced by the thermodynamic limit $\mathcal{A}^{[\beta]}$ rather than with periodic or monochromatic boundary conditions, we have to explicitly rule out the situation where the phase separating curves cross or go along the boundary of the considered finite volume region $\mathbb{B}_{2}(L)$. To this end, for $\alpha>0$ shall write $\mathcal{N}[\alpha, L]$ for the event that no $\alpha$-large contour of $\mathcal{A}^{[\beta]}$ gets closer than at the distance $6 \alpha$ to the circle $\mathbb{S}_{1}(L):=\partial \mathbb{B}_{2}(L)$. In what follows we shall write

$$
\alpha[L]:=\sqrt{L} \log L
$$

Our main result is the following theorem.

Theorem 1.2 For $0<a<2 \pi|\mathbb{M}[\beta]|$ we have

$$
\begin{gathered}
\mathbb{P}\left(\mathbb{M}_{L}\left(\mathcal{A}^{[\beta]}\right) \geq \mathbb{M}[\beta] \pi L^{2}+a L^{2}, \mathcal{N}[\alpha[L], L] \text { holds }\right)=\exp \left(-\sqrt{\frac{2 \pi a}{|\mathbb{M}[\beta]|}} L \tau_{\alpha[L]}^{[\beta]}+O(\alpha[L])\right) \\
=\exp \left(-\sqrt{\frac{2 \pi a}{|\mathbb{M}[\beta]|}} L \tau^{[\beta]}+o(L)\right) .
\end{gathered}
$$

Moreover, there exists a constant $C_{\text {large }}$ such that on the event

$$
\left\{\mathbb{M}_{L}\left(\mathcal{A}^{[\beta]}\right) \geq \mathbb{M}[\beta] \pi L^{2}+a L^{2}, \mathcal{N}[\alpha[L], L] \text { holds }\right\}
$$


for sufficiently large $L$ we have with probability arbitrarily close to 1

- There is exactly one $C_{\text {large }} \log L$-large contour $\theta_{\text {large }}$,

- This $\theta_{\text {large }}$ satisfies

$$
\min _{x} \rho_{H}\left(\theta_{\text {large }}, \mathbb{S}_{1}\left(x, L \sqrt{\frac{a}{2 \pi|\mathbb{M}[\beta]|}}\right)\right)=O\left(L^{3 / 4} \sqrt{\log L}\right),
$$

with $\rho_{H}$ standing for the usual Hausdorff distance.

Note that in the sequel we shall refer to the condition $\mathbb{M}_{L}\left(\mathcal{A}^{[\beta]}\right) \geq \mathbb{M}[\beta] \pi L^{2}+a L^{2}$ as to the micro-canonical constraint.

The remaining part of the paper is the proof of Theorem 1.2 and is organised as follows. In Section 2 below we establish upper bounds on occurrence probabilities of large contours. Next, in Section 3 we study moderate deviation probabilities for cut-off contour ensembles of polygonal fields. Section 4 provides a simple yet important lemma allowing us to factorise the avoidance probabilities of $\mathcal{A}^{[\beta]}$ over disjoint regions. This is followed by Section 5 dealing with properties of the surface tension, and then by Section 6, where we establish coarsegraining skeleton estimates. The complementary lower bounds for occurrence probabilities of large contours are stated in Section 7. Finally, in Section 8 we complete the proof of Theorem 1.2 by putting together the results of previous sections.

\section{Exponential tightness bounds}

In this section we show that although the total length of the contour ensemble $\mathcal{A}^{[\beta]} \cap \mathbb{B}_{2}(L)$ is clearly of the area order $\Theta\left(L^{2}\right)$, this is due to the contributions of $O(\log L)$-small contours, while the contribution of $\Omega(\log L)$-large contours is of order $O(1)$ with the corresponding large deviation probabilities exhibiting exponential decay. To put it in formal terms, with $\alpha>0$ not necessarily given by (1.16), write $\mathbb{L}_{\alpha, L}:=\mathbb{L}_{\alpha, L}\left[\mathcal{A}^{[\beta]}\right]$ for the family of $\alpha$-large contours of $\mathcal{A}^{[\beta]}$ hitting $\mathbb{B}_{2}(L)$ and, in general, let $\mathbb{L}_{\alpha, L}[\gamma]$ stand for the family of $\alpha$-large contours of a contour collection $\gamma$ which hit $\mathbb{B}_{2}(L)$. We claim that

Lemma 2.1 For each $\kappa<\beta / 2-2$ there exist $M, C=C(\beta, \kappa)<\infty$ such that for all $\alpha>C \log L$ and $\lambda>0$ we have

$$
\mathbb{P}\left(\text { length }\left(\mathbb{L}_{\alpha, L}\right)>\lambda\right) \leq M \exp (-\kappa \lambda)
$$


and the same applies for $\mathbb{L}_{\alpha, L}$ replaced with $\mathbb{L}_{\alpha, L}\left[\mathcal{A}^{[\beta] ; \alpha, \cdot}\right], \mathbb{L}_{\alpha, L}\left[\mathcal{A}_{(\cdot):(\cdot)}^{[\beta] ; \cdot(\cdot)}\right]$ and $\mathbb{L}_{\alpha, L}\left[\mathcal{A}_{(\cdot)}^{[\beta, h] ; \alpha,(\cdot)}\right]$, $\mathbb{L}_{\alpha, L}\left[\mathcal{A}_{(\cdot):(\cdot)}^{[\beta, h] ; \alpha,(\cdot)}\right]$ for $h$ within the validity range of $(1.9)$.

Note that it is natural to regard this lemma as an exponential tightness statement for length $\left(\mathbb{L}_{\alpha, L}\right)$, whence the title of the section.

Proof We provide the proof for the polygonal field $\mathcal{A}^{[\beta]}$ only, since the argument goes exactly along the same lines for all the modified fields obtained from the variants of the graphical construction discussed in Subsubsection 1.2.2 and admitting stochastically dominating Poisson contour processes. Note that the assumption $\kappa<\beta / 2-2$ was imposed for the purpose of dealing with area-interacting processes with the external field $h$ within the validity range of (1.9), which admit stochastic domination by the Poisson contour process $\mathcal{P}_{\Theta[\beta / 2]}$. For the remaining polygonal fields considered in the statement of the lemma, with no area interaction, a stronger stochastic domination by $\mathcal{P}_{\Theta[\beta]}$ is available and the assertion of the lemma still holds if we choose $\kappa<\beta-2$ rather than $\kappa<\beta / 2-2$.

To proceed, use the graphical construction to conclude that the total length of contours in $\mathbb{L}_{\alpha, L}$ is stochastically bounded by the total length of $\alpha$-large contours of $\mathcal{P}_{\Theta^{[\beta / 2]}}$ hitting $\mathbb{L}_{\alpha, L}$. Thus, by the definition of a Poisson point process,

$$
\begin{aligned}
\mathbb{E} \exp \left(\kappa \operatorname{length}\left(\mathbb{L}_{\alpha, L}\right)\right) \leq \exp & {\left[\int_{\left\{\theta \in \mathcal{C} \mid \theta \cap \mathbb{B}_{2}(L) \neq \emptyset, \theta \text { is } \alpha \text {-large }\right\}}\left(\mathrm{e}^{\kappa \operatorname{length}(\theta)}-1\right) d \Theta^{[\beta / 2]}(\theta)\right] \leq } \\
& \mathrm{e}^{\kappa \alpha} \zeta(\alpha)+\kappa \int_{\alpha}^{\infty} \mathrm{e}^{\kappa \lambda} \zeta(\lambda) d \lambda,
\end{aligned}
$$

where the last inequality follows by integration by parts with

$$
\zeta(\lambda):=\Theta^{[\beta / 2]}\left(\left\{\theta \in \mathcal{C} \mid \theta \cap \mathbb{B}_{2}(L) \neq \emptyset, \text { length }(\theta)>\lambda\right\}\right) .
$$

In view of Lemma 1.1 this means that

$$
\mathbb{E} \exp \left(\kappa \operatorname{length}\left(\mathbb{L}_{\alpha, L}\right)\right) \leq \exp \left[C_{1}(\beta, \kappa) L^{2} \exp \left(\left[\kappa+2-\frac{\beta}{2}\right] \alpha\right)\right]
$$

with some constant $C_{1}(\beta, \kappa)$. Thus, using Markov inequality we get

$$
\mathbb{P}\left(\text { length }\left(\mathbb{L}_{\alpha, L}\right)>\lambda\right) \leq \frac{\mathbb{E} \exp \left(\kappa \operatorname{length}\left(\mathbb{L}_{\alpha, L}\right)\right)}{\exp (\kappa \lambda)} \leq \exp \left[C_{1}(\beta, \kappa) L^{2} \exp \left(\left[\kappa+2-\frac{\beta}{2}\right] \alpha\right)\right] \mathrm{e}^{-\kappa \lambda}
$$

which completes the proof of the lemma for $\alpha>C \log L$ with large enough $C=C(\beta, \kappa)$. 


\section{Moderate deviations for cut-off ensembles}

The current section deals with the properties of the cut-off ensembles $\mathcal{A}^{[\beta] ; \alpha, V}$ arising by conditioning the original field $\mathcal{A}^{[\beta]}$ on containing no $\alpha$-large contours hitting $V \subseteq \mathbb{R}^{2}$. Recall that we assume here that $\beta>\beta_{g}$ and $\beta>\hat{\beta}_{g}$ so that $\beta$ falls into the validity regions of the graphical construction discussed in Subsection 1.2 as well as of its area-interacting modification discussed in Subsubsection 1.2.2. We consider $\alpha$ not necessarily given by (1.16). Our first observation is that imposing a cut-off does not change the expected magnetisation by too much

$$
\left|\pi L^{2} \mathbb{M}[\beta]-\mathbb{E} \mathbb{M}_{L}\left(\mathcal{A}^{[\beta] ; \alpha, \mathbb{B}_{2}(L)}\right)\right|=O\left(L^{4} \exp (-c \alpha)\right)
$$

with some $c>0$. Indeed, this follows by the fact that, in view the stochastic domination of $\mathcal{A}^{[\beta]}$ by $\mathcal{P}_{\Theta[\beta]}$ and in view of Lemma 1.1, an $\alpha$-large contour shows up in $\mathcal{A}^{[\beta]} \cap \mathbb{B}_{2}(L)$ with probability $O\left(L^{2} \exp (-c \alpha)\right)$, whence conditioning on the absence of this event can change the probabilities of other events by at most $O\left(L^{2} \exp (-c \alpha)\right)$, consequently the variational distance between the laws $\mathcal{L}\left(\mathcal{A}^{[\beta]}\right)$ and $\mathcal{L}\left(\mathcal{A}^{[\beta] ; \alpha, \mathbb{B}_{2}(L)}\right)$ is of the same order $O\left(L^{2} \exp (-c \alpha)\right)$. To get (3.18) it suffices now to observe that the magnetisation over $\mathbb{B}_{2}(L)$ is a.s. bounded in absolute value by $\pi L^{2}$.

Another useful observation is that the impact of imposing a forbidden region for cut-off ensembles can also be very well controlled. In formal terms, we claim that for a collection $\gamma$ of $\alpha$-large contours, $\alpha>1$, in $\mathbb{B}_{2}(L)$ we have

$$
\left|\mathbb{E} \mathbb{M}_{L}\left(\mathcal{A}^{[\beta] ; \alpha, \mathbb{B}_{2}(L)}\right)-\mathbb{E} \mathbb{M}_{L}\left(\mathcal{A}_{\mathbb{R}^{2}: \gamma}^{[\beta] ; \alpha \mathbb{B}_{2}(L)}\right)\right|=O\left(\operatorname{Area}\left(\gamma \oplus \mathbb{B}_{2}(1)\right)\right)=O(\operatorname{length}(\gamma)) .
$$

This is an immediate consequence of the fact that, by (1.7), under the canonical coupling of $\mathcal{A}^{[\beta] ; \alpha, \mathbb{B}_{2}(L)}$ and $\mathcal{A}_{\mathbb{R}^{2}: \gamma}^{[\beta] \alpha, \mathbb{B}_{2}(L)}$ the probability that the colours assigned to a given point $x$ by these ensembles differ, is of order $O(\exp (-c \operatorname{dist}(x, \gamma))), c>0$.

The argument leading to (3.18) and (3.19) above can be easily modified to yield the following combination of these relations. Let $\gamma$ be a collection of $\alpha$-large contours in $\mathbb{B}_{2}(L)$. Then

$$
\left|\mathbb{E} \mathbb{M}_{L}\left(\mathcal{A}_{\mathbb{R}^{2} ; \gamma}^{[\beta] \alpha, \mathbb{B}_{2}(L)} \cup \gamma\right)-\right| \mathbb{M}[\beta]\left|\mathbb{M}_{L}(\gamma)\right|=O(\operatorname{length}(\gamma))
$$

provided $\alpha \geq C \log L$ for sufficiently large $C$.

The main result of this section is the following moderate deviation bound 
Theorem 3.1 For each $\beta$ large enough there exists a positive constant $C_{1}=C_{1}(\beta)$ such that, uniformly in $L, \alpha \geq C_{1} \log L$ and in finite collections $\gamma$ of polygonal contours in $\mathbb{R}^{2}$ we have for all $0<A \leq C_{1}^{-1} L^{2} / \log L$

$$
\mathbb{P}\left(\left|\mathbb{M}_{L}\left(\mathcal{A}_{\mathbb{R}^{2}: \gamma}^{[\beta] ;, \mathbb{B}_{2}(L)} \cup \gamma\right)-\mathbb{E}_{L}\left(\mathcal{A}_{\mathbb{R}^{2} ; \gamma}^{[\beta] ; \mathbb{B}_{2}(L)} \cup \gamma\right)\right|>A\right) \leq \exp \left(-c\left[\frac{A^{2}}{L^{2}} \wedge \frac{A}{\alpha}\right]\right)
$$

with some constant $c>0$.

\section{Proof Write}

$$
\mu_{L, \gamma}^{\alpha}:=\mathbb{M}_{L}\left(\mathcal{A}_{\mathbb{R}^{2}: \gamma}^{\left[[] ; \alpha, \mathbb{B}_{2}(L)\right.} \cup \gamma\right)
$$

and let

$$
\mu_{L}^{\alpha}=\mu_{L, \emptyset}^{\alpha}=\mathbb{M}_{L}\left(\mathcal{A}^{[\beta] ; \alpha, \mathbb{B}_{2}(L)}\right), \mu_{L}=\mu_{L}^{\infty}=\mathbb{M}_{L}\left(\mathcal{A}^{[\beta]}\right) .
$$

For $h \in \mathbb{R}$ consider the partition function

$$
Z[h]:=\mathbb{E} \exp \left(h \mu_{L, \gamma}^{\alpha}\right)
$$

The following estimate, valid for all $h$ satisfying (1.9), is the crucial ingredient of our proof:

$$
\log Z[h] \leq h \mathbb{E} \mu_{L, \gamma}^{\alpha}+h^{2} L^{2} \sigma^{2} / 2
$$

for some $\sigma>0$, uniformly in $L, \gamma, \alpha$ and $h$ within the validity region of (1.9). To see that (3.21) suffices to complete the proof of the theorem, take first $0<A \leq \frac{\beta L^{2} \sigma^{2}}{\pi^{2} \alpha}$, put $h:=\frac{A}{\sigma^{2} L^{2}}$ which clearly satisfies (1.9), and then use Markov's inequality to conclude that

$$
\mathbb{P}\left(\mu_{L, \gamma}^{\alpha}>\mathbb{E} \mu_{L, \gamma}^{\alpha}+A\right) \leq \frac{Z[h]}{\exp \left(h\left(\mathbb{E} \mu_{L, \gamma}^{\alpha}+A\right)\right)} \leq \exp \left(h^{2} L^{2} \sigma^{2} / 2-A h\right)=\exp \left(-\frac{A^{2}}{2 \sigma^{2} L_{(3.22)}^{2}}\right) .
$$

Next, for $A>\frac{\beta L^{2} \sigma^{2}}{\pi^{2} \alpha}$ choose $\kappa<\beta / 2-2$ and $C(\beta, \kappa)$ as in Lemma 2.1 and assume that $C_{1}$ in the present lemma is chosen so that $\tilde{\alpha}:=2 C(\beta, \kappa) \log L<\alpha$. Then, on the event $\left\{\mu_{L, \gamma}^{\alpha}>\mathbb{E} \mu_{L, \gamma}^{\alpha}+A\right\}$ there are two possible scenarios:

- The total length of $\tilde{\alpha}$-large contours in $\mathbb{L}_{\tilde{\alpha}, L}\left(\mathcal{A}_{\mathbb{R}^{2}: \gamma}^{[\beta] ;, \mathbb{B}_{2}(L)}\right)$ exceeds $\frac{2 A}{\pi A}$, which can happen with probability at most $M \exp \left(-\frac{2 \kappa A}{\pi \alpha}\right)$ by the exponential tightness Lemma 2.1 , 
- The total length of $\tilde{\alpha}$-large contours in $\mathbb{L}_{\tilde{\alpha}, L}\left(\mathcal{A}_{\mathbb{R}^{2}: \gamma}^{[\beta] ;, \mathbb{B}_{2}(L)}\right)$ does not exceed $\frac{2 A}{\pi \alpha}$. Since $\frac{8}{\pi \alpha}$ is the lower bound for the length-to-enclosed-area ratio for an $\alpha$-small contour, this means in particular that the total area enclosed by $\tilde{\alpha}$-large contours of $\mathcal{A}_{\mathbb{R}^{2}: \gamma}^{[\beta] ; \alpha, \mathbb{B}_{2}(L)}$ falls below $A / 4$. Denoting by $\tilde{\gamma}$ the family of contours $\mathbb{L}_{\tilde{\alpha}, L}\left(\mathcal{A}_{\mathbb{R}^{2}: \gamma}^{[\beta] ;, \mathbb{B}_{2}(L)}\right) \cup \gamma$ and taking into account that the change of magnetisation induced by adding a contour is bounded in absolute value by twice the area it encloses, conditionally on given $\tilde{\gamma}$, we are led to

$$
\mathbb{E} \mu_{L, \tilde{\gamma}}^{\tilde{\alpha}} \leq \mathbb{E M}_{L}\left(\mathcal{A}_{\mathbb{R}^{2}: \tilde{\gamma}}^{[\beta] \tilde{\alpha}, \mathbb{B}_{2}(L)} \cup \gamma\right)+A / 2
$$

Now, in full analogy with (3.19), on the considered event we get

$$
\left|\mathbb{E M}_{L}\left(\mathcal{A}_{\mathbb{R}^{2}: \tilde{\gamma}}^{[\beta] ; \tilde{B}}(L) \cup \gamma\right)-\mathbb{E} \mu_{L, \gamma}^{\tilde{\alpha}}\right|=O\left(\frac{2 A}{\pi \alpha}\right) .
$$

Next, in full analogy with (3.18), we have

$$
\left|\mathbb{E} \mu_{L, \gamma}^{\tilde{\alpha}}-\mu_{L, \gamma}^{\alpha}\right|=O\left(L^{4} \exp (-c \tilde{\alpha})\right)
$$

which goes to 0 faster than the inverse of any polynomial under appropriate choice of $C(\beta, \kappa)$ in Lemma 2.1. Putting the above relations together we conclude that

$$
\mathbb{E} \mu_{L, \tilde{\gamma}}^{\tilde{\alpha}} \leq \mathbb{E} \mu_{L, \gamma}^{\alpha}+A / 2(1+o(1))
$$

Recalling that $\mu_{L, \gamma}^{\alpha}$ given $\tilde{\gamma}$ coincides in law with $\mu_{L, \tilde{\gamma}}^{\tilde{\alpha}}$, we are led to

$$
\mathbb{P}\left(\mu_{L, \gamma}^{\alpha}>\mathbb{E}_{L, \gamma}^{\alpha} \mid \tilde{\gamma}\right) \leq \mathbb{P}\left(\mu_{L, \tilde{\gamma}}^{\tilde{\alpha}}>\mathbb{E}_{L, \tilde{\gamma}}^{\tilde{\alpha}}+A / 2(1-o(1))\right)
$$

Now, choosing $C_{1}$ so that $A \leq \frac{\beta L^{2} \sigma^{2}}{\pi^{2} \tilde{\alpha}}$, we can bound above the last probability by $\exp \left(-\frac{A^{2}}{8 \sigma^{2} L^{2}}\right)$ applying (3.22) with $\alpha$ and $\gamma$ replaced there by $\tilde{\alpha}$ and $\tilde{\gamma}$ respectively.

Combining the above two points with (3.22) and noting that the probability of $\left\{\mu_{L, \gamma}^{\alpha}<\right.$ $\left.\mathbb{E} \mu_{L, \gamma}^{\alpha}-A\right\}$ can be dealt with in a completely analogous way, we obtain the assertion of the theorem.

It remains to verify (3.21) for $h$ satisfying (1.9). We extend the notation by putting

$$
\mu_{L, \gamma}^{\alpha, h}:=\mathbb{M}_{L}\left(\mathcal{A}_{\mathbb{B}_{2}(L): \gamma}^{[\beta, h] ; \alpha, \mathbb{B}_{2}(L)} \cup \gamma\right)
$$

Noting that

$$
\frac{\partial}{\partial h} \log Z[h]=\mathbb{E} \mu_{L, \gamma}^{\alpha, h}, \frac{\partial^{2}}{\partial h^{2}} \log Z[h]=\operatorname{Var}\left[\mu_{L, \gamma}^{\alpha, h}\right]
$$


and Taylor expanding the logarithm of the partition function up to the second order term yields

$$
\log Z[h]=h \mathbb{E} \mu_{L, \gamma}^{\alpha}+h^{2} \frac{\operatorname{Var}\left[\mu_{L, \gamma}^{\alpha, h^{*}}\right]}{2}
$$

for some $h^{*}$ between 0 and $h$. We claim that, uniformly in $L, \alpha, \gamma$ and $h$ satisfying (1.9), the variance $\operatorname{Var}\left[\mu_{L, \gamma}^{\alpha, h}\right]$ is of the area order $O\left(L^{2}\right)$

$$
\operatorname{Var}\left[\mu_{L, \gamma}^{\alpha, h}\right]=O\left(L^{2}\right)
$$

which, once established, will immediately yield the required relation (3.21) as a conclusion of (3.24). To prove (3.25) we show that, for $U_{1}, U_{2} \subseteq \mathbb{B}_{2}(L)$, uniformly in $\gamma$ and in $h$ satisfying (1.9)

$$
\begin{gathered}
\operatorname{Cov}\left[\mathbb{M}_{U_{1}}\left(\mathcal{A}_{\mathbb{B}_{2}(L): \gamma}^{[\beta, h] ; \alpha, \mathbb{B}_{2}(L)} \cup \gamma\right) ; \mathbb{M}_{U_{2}}\left(\mathcal{A}_{\mathbb{B}_{2}(L): \gamma}^{[\beta, h] ; \alpha, \mathbb{B}_{2}(L)} \cup \gamma\right)\right]= \\
O\left(\operatorname{Area}\left(U_{1}\right) \operatorname{Area}\left(U_{2}\right)\left[\operatorname{Area}\left(U_{1} \oplus \mathbb{B}_{2}(1)\right)+\operatorname{Area}\left(U_{2} \oplus \mathbb{B}_{2}(1)\right)\right] \mathrm{e}^{-c \operatorname{dist}\left(U_{1}, U_{2}\right)}\right)
\end{gathered}
$$

for a positive constant $c$, with $\oplus$ standing for the usual Minkowski addition. Indeed, with the representation provided by the graphical construction for area-interacting fields in Subsubsection 1.2.2, conditionally on the event $\left\{\hat{\mathrm{An}_{0}}\left(U_{1}\right) \subseteq U_{1} \oplus \mathbb{B}_{2}\left(\frac{\operatorname{dist}\left(U_{1}, U_{2}\right)}{2}\right), \hat{\mathrm{An}_{0}}\left(U_{2}\right) \subseteq\right.$ $\left.U_{2} \oplus \mathbb{B}_{2}\left(\frac{\operatorname{dist}\left(U_{1}, U_{2}\right)}{2}\right)\right\}$ the random variables $\mathbb{M}_{U_{1}}\left(\mathcal{A}_{\mathbb{B}_{2}(L): \gamma}^{[\beta, h] ; \alpha, \mathbb{B}_{2}(L)} \cup \gamma\right)$ and $\mathbb{M}_{U_{2}}\left(\mathcal{A}_{\mathbb{B}_{2}(L): \gamma}^{[\beta, h] ; \alpha, \mathbb{B}_{2}(L)} \cup \gamma\right)$ are independent. But in view of (1.10) the probability of this event does not fall below $1-O\left(\left[\operatorname{Area}\left(U_{1} \oplus \mathbb{B}_{2}(1)\right)+\operatorname{Area}\left(U_{2} \oplus \mathbb{B}_{2}(1)\right)\right] \exp \left(-c \operatorname{dist}\left(U_{1}, U_{2}\right)\right)\right)$. This observation combined with the fact that $\left|\mathbb{M}_{U_{i}}\left(\mathcal{A}_{\mathbb{B}_{2}(L): \gamma}^{[\beta, h] ;, \mathbb{B}_{2}(L)}\right)\right| \leq \operatorname{Area}\left(U_{i}\right), i=1,2$ implies (3.26). The required relation (3.25) follows now from (3.26) by usual argument based on splitting $\mathbb{B}_{2}(L)$ into $\Theta\left(L^{2}\right)$ disjoint regions of diameter and area $\Theta(1)$ and then noting that, with the magnetisation contributions coming from distant regions exhibiting exponentially decaying covariances, the asymptotic order of the total magnetisation variance $\operatorname{Var}\left[\mu_{L, \gamma}^{\alpha, h}\right]$ is determined by the sum of covariances between regions within distance $\Theta(1)$ from each other, which yields the desired order $O\left(L^{2}\right)$. The proof is complete.

Remark 3.2 We note that the bounds in Theorem 3.1 are of optimal order only for the probabilities of positive deviations $\left\{\mu_{L, \gamma}^{\alpha}>\mathbb{E} \mu_{L, \gamma}^{\alpha}+A\right\}, A>0$. We believe that the probabilities of negative moderate deviations $\left\{\mu_{L, \gamma}^{\alpha}<\mathbb{E} \mu_{L, \gamma}^{\alpha}-A\right\}$ as well as $\left\{\mu_{L}<\mathbb{E} \mu_{L}-A\right\}$, $A \ll$ 
$L^{2}$, exhibit Gaussian-type decay $\exp \left(-\Omega\left(A^{2} / L^{2}\right)\right)$ as in classical moderate deviation regime, in full analogy with similar phenomenon for the Ising model, see [8], (1.1.2), (2.3.2) in [12] or Section III.C.1 in [6] and the references therein. Since this falls beyond the context of our further argument, we do not discuss this issue in the present paper.

As an easy corollary from Theorem 3.1 we conclude that

Corollary 3.3 With $A \geq 1, \alpha$ and $\gamma$ as in Theorem 3.1 and with $C_{1}$ in Theorem 3.1 large enough we have uniformly

$\mathbb{P}\left(\left|\mathbb{M}_{L}\left(\mathcal{A}_{\mathbb{R}^{2}: \gamma}^{[\beta]} \cup \gamma\right)-\mathbb{E} \mathbb{M}_{L}\left(\mathcal{A}_{\mathbb{R}^{2}: \gamma}^{[\beta]} \cup \gamma\right)\right|>A\right) \leq \exp \left(-c\left[\frac{A^{2}}{L^{2}} \wedge \frac{A}{\alpha}\right]\right) \vee O\left(L^{2} \exp (-c \alpha)\right)$

with some constant $c>0$.

Proof This is a direct conclusion of Theorem 3.1 combined with the observation that the variational distance between the laws $\mathcal{L}\left(\mathcal{A}_{\mathbb{R}^{2} ; \gamma}^{[\beta]} \cap \mathbb{B}_{2}(L)\right)$ and $\mathcal{L}\left(\mathcal{A}_{\mathbb{R}^{2} ; \gamma}^{[\beta] ;, \mathbb{B}_{2}(L)} \cap \mathbb{B}_{2}(L)\right)$ is of order $O\left(L^{2} \exp (-c \alpha)\right)$, in full analogy with the argument leading to (3.18) above.

Another useful corollary relies on a straightforward observation that the proof of Theorem 3.1 applies with only minor modifications for $\mathcal{A}^{[\beta] ; \alpha, \mathbb{B}_{2}(L)}$ replaced by $\mathcal{A}_{\mathbb{B}_{2}(L)}^{[\beta, h] ;, \mathbb{B}_{2}(L)}$ with $|h| \leq H / \alpha, H$ small enough. In formal terms,

Corollary 3.4 With $H>0$ small enough, for each $\beta$ large enough there exists a positive constant $C=C(\beta, H)$ such that, uniformly in $L, \alpha \geq C \log L$, finite collection $\gamma$ of polygonal contours in $\mathbb{R}^{2}$ and $|h| \leq H / \alpha$, we have for all $0<A \leq C^{-1} L^{2} / \log L$

$$
\mathbb{P}\left(\left|\mathbb{M}_{L}\left(\mathcal{A}_{\mathbb{B}^{2}(L): \gamma}^{[\beta, h], \alpha, \mathbb{B}_{2}(L)} \cup \gamma\right)-\mathbb{E} \mathbb{M}_{L}\left(\mathcal{A}_{\mathbb{B}^{2}(L): \gamma}^{[\beta, h] ;, \mathbb{B}_{2}(L)} \cup \gamma\right)\right|>A\right) \leq \exp \left(-c\left[\frac{A^{2}}{L^{2}} \wedge \frac{A}{\alpha}\right]\right)
$$

with some constant $c>0$.

We omit the proof of this corollary which is just a simple repetition of the proof of Theorem 3.1 .

Below, we provide some further auxiliary results related to moderate deviation probabilities for cut-off ensembles. Note first that we can establish a bound analogous to (3.25) for the third cumulant of $\mu_{L, \gamma}^{\alpha, h}$ :

$$
\frac{\partial^{3}}{\partial h^{3}} \log Z[h]=O\left(L^{2}\right)
$$


uniformly in $\alpha, L$, finite contour collection $\gamma$ and $h$ satisfying (1.9). We omit the details of the argument, based on the relation (1.10), since it goes along the same lines as the proof of Lemma 5.3 in Baryshnikov \& Yukich [5] (valid for arbitrary order cumulants in fact). In particular, in view of (3.23) combined with (3.27), we get for $h$ within the validity range of (1.9)

$$
\mathbb{E} \mu_{L, \gamma}^{\alpha, h}=\mathbb{E} \mu_{L, \gamma}^{\alpha}+h \operatorname{Var}\left[\mu_{L, \gamma}^{\alpha}\right]+O\left(h^{2} L^{2}\right)
$$

uniformly in $L, \gamma$. To proceed, assume that $\gamma$ is a finite contour collection in $\mathbb{B}_{2}(L)$ with $\operatorname{Area}\left(\gamma \oplus \mathbb{B}_{2}\left(\log ^{2} L\right)\right) \leq L^{2} / \log L$. We claim that under this condition we have (3.25) strengthened to

$$
\operatorname{Var}\left[\mu_{L, \gamma}^{\alpha}\right]=\Theta\left(L^{2}\right)
$$

uniformly in $\gamma, L$. Indeed, observe that by (1.7) the probability of the event $\left\{\operatorname{An}_{0}(\gamma) \not\right.$ $\left.\gamma \oplus \mathbb{B}_{2}\left(\log ^{2} L\right)\right\}$ is of order at most $O\left(L^{2} \exp \left(-c \log ^{2} L\right)\right), c>0$, whence under the canonical coupling with probability $1-O\left(L^{2} \exp \left(-c \log ^{2} L\right)\right)$ the field $\mathcal{A}_{\mathbb{R}^{2}: \gamma}^{[\beta] ; \mathbb{B}_{2}(L)}$ coincides with $\mathcal{A}^{[\beta] ; \alpha, \mathbb{B}_{2}(L)}$ over the whole complement of $\gamma \oplus \mathbb{B}_{2}\left(\log ^{2} L\right)$. Consequently,

$$
\left|\operatorname{Var}\left[\mu_{L}^{\alpha}\right]-\operatorname{Var}\left[\mu_{L, \gamma}^{\alpha}\right]\right|=o\left(L^{2}\right) .
$$

Now, mimicking the proof of (3.18) we check that

$$
\left|\operatorname{Var}\left[\mu_{L}^{\alpha}\right]-\operatorname{Var}\left[\mu_{L}\right]\right|=O\left(L^{6} \exp (-c \alpha)\right)=o\left(L^{2}\right)
$$

provided $\alpha \geq C \log L$ with $C$ large enough. This will yield the required relation (3.29) as soon as we show that for the field $\mathcal{A}^{[\beta]}$ the variance of magnetisation has the required order

$$
\operatorname{Var}\left[\mu_{L}\right]=\Omega\left(L^{2}\right)
$$

To this end, we fix some large $\lambda>0$, large $k \in \mathbb{N}$ and small $\epsilon>0$ and we note that

$\inf \left\{\operatorname{Var}\left[\mathbb{M}_{D}\left(\mathcal{A}_{D}^{[\beta]}\right)\right] \mid \operatorname{Area}(D) \geq \epsilon \lambda^{2}, \operatorname{card} \operatorname{Vertices}(D) \leq k, \operatorname{length}(\partial D) \in\left[\epsilon \lambda, \epsilon^{-1} \lambda\right]\right\}>0$,

with the infimum taken over all bounded domains $D$ with polygonal boundary, possibly chopped off by intersecting with $\mathbb{B}_{2}(L)$. Indeed, this can be proven by observing first that the mapping $D \mapsto \phi(D):=\operatorname{Var}\left[\mathbb{M}_{D}\left(\mathcal{A}_{D}^{[\beta]}\right)\right]$ admits only strictly positive values and it is 
continuous with respect to the pseudo-metric $\rho_{H}^{*}\left(D_{1}, D_{2}\right):=\inf _{x \in \mathbb{R}^{2}} \rho_{H}\left(D_{1}, x+D_{2}\right)$. Thus, putting $\mathcal{D}[\lambda, k, \epsilon]:=\left\{D \mid \operatorname{Area}(D) \geq \epsilon \lambda^{2}, \operatorname{card} \operatorname{Vertices}(D) \leq k, \operatorname{length}(\partial D) \in\left[\epsilon \lambda, \epsilon^{-1} \lambda\right]\right\}$ and noting that $\mathcal{D}[\lambda, k, \epsilon]$ is compact in $\rho_{H}^{*}$, we see that $\phi_{0}:=\inf _{D \in \mathcal{D}[\lambda, k, \epsilon]} \phi(D)>0$, which yields the required relation (3.31). To proceed, note that the variance $\operatorname{Var}\left[\mu_{L}\right]$ in $(3.30)$ is bounded below by the expectation of the conditional variance of $\mu_{L}$ given the ensemble of external (outermost) contours $\theta$ in $\mathcal{A}^{[\beta]} \cap \mathbb{B}_{2}(L)$ satisfying the constraints of the infimum in (3.31) for $D:=\operatorname{Int} \theta$. Thus, taking into account that given the presence of such $\theta$ the behaviour of the process $\mathcal{A}^{[\beta]}$ inside $\theta$ is independent of that outside $\theta$ and then using (3.31) to conclude that each such $\theta$ present brings a contribution of at least $\phi_{0}$ to the considered conditional variance, we have $\operatorname{Var}\left[\mu_{L}\right]$ bounded below by $\phi_{0}$ times the expected number of external (outermost) contours $\theta$ in $\mathcal{A}^{[\beta]} \cap \mathbb{B}_{2}(L)$ as in (3.31) with $D=\operatorname{Int} \theta$. Since this number is clearly of the area order $\Omega\left(L^{2}\right)$, the required relation (3.30) has been established, which completes the argument for (3.29).

Putting together (3.28), (3.29) and the observations that $h=o(1)$ by (1.9) and that $\mathbb{E} \mu_{L, \gamma}^{\alpha, h}$ strictly increases with $h$ we come to

Corollary 3.5 There are positive constants $K_{0}=K_{0}(\beta)$ and $C=C(\beta)$ such that for each $\alpha \geq C \log L$, each $\Delta$ with $|\Delta| \leq K_{0} L^{2} / \alpha$ and each finite contour collection $\gamma$ with $\operatorname{Area}\left(\gamma \oplus \mathbb{B}_{2}\left(\log ^{2} L\right)\right) \leq L^{2} / \log L$ there exists a unique value $h=h[\Delta, L, \gamma]$ of external magnetic field such that

$$
\mathbb{E} \mu_{L, \gamma}^{\alpha, h}=\mathbb{E} \mu_{L, \gamma}^{\alpha}+\Delta
$$

and

$$
h=\Theta\left(\Delta / L^{2}\right)
$$

uniformly in $\alpha, \Delta, L, \gamma$.

Our next statement provides a lower bound for moderate deviation probabilities of $\mu_{L, \gamma}^{\alpha}$, complementary to the upper bound of Theorem 3.1.

Lemma 3.6 For all $0 \leq \Delta \ll L^{2} / \alpha$, with $\alpha$ and $\gamma$ as in Corollary 3.5 and with $\alpha \ll L /$ $\log L$ we have uniformly in $\Delta, \alpha, L, \gamma$

$$
\mathbb{P}\left(\mu_{L, \gamma}^{\alpha}>\mathbb{E} \mu_{L, \gamma}^{\alpha}+\Delta\right) \geq \exp \left(-O\left([\Delta+L \log L]^{2} / L^{2}\right)\right)
$$


Proof Write using Corollary 3.5, putting for brevity $h[\cdot]:=h[\cdot, L, \gamma]$,

$$
\begin{aligned}
& \mathbb{P}\left(\mu_{L, \gamma}^{\alpha}>\mathbb{E} \mu_{L, \gamma}^{\alpha}+\Delta\right) \geq \mathbb{P}\left(\left|\mu_{L, \gamma}^{\alpha}-\mathbb{E} \mu_{L, \gamma}^{\alpha}-\Delta-L \log L\right|<L \log L\right) \geq \\
& \exp \left(-h[\Delta+L \log L]\left[\mathbb{E} \mu_{L, \gamma}^{\alpha}+\Delta+2 L \log L\right]\right) \mathbb{E} \exp \left(h[\Delta+L \log L] \mu_{L, \gamma}^{\alpha}\right) \\
& \mathbb{P}\left(\left|\mu_{L, \gamma}^{\alpha, h[\Delta+L \log L]}-\mathbb{E} \mu_{L, \gamma}^{\alpha, h[\Delta+L \log L]}\right|<L \log L\right)
\end{aligned}
$$

and use Jensen's inequality to bound it below by

$$
\mathbb{P}\left(\left|\mu_{L, \gamma}^{\alpha, h[\Delta+L \log L]}-\mathbb{E} \mu_{L, \gamma}^{\alpha, h[\Delta+L \log L]}\right|<L \log L\right) \exp (-h[\Delta+L \log L][\Delta+2 L \log L]) .
$$

Thus, taking into account that $h[\Delta+L \log L]=\Theta\left([\Delta+L \log L] / L^{2}\right)$ by Corollary 3.5 and that $\mathbb{P}\left(\left|\mu_{L, \gamma}^{\alpha, h[\Delta+L \log L]}-\mathbb{E} \mu_{L, \gamma}^{\alpha, h[\Delta+L \log L]}\right|<L \log L\right)=1-o(1)$ in view of Corollary 3.4, completes the proof of the lemma.

\section{Decoupling lemma}

The purpose of this section is to establish Lemma 4.1 stating that the avoidance probabilities for the field $\mathcal{A}^{[\beta]}$ over disjoint regions can be very well approximated by the product of the corresponding avoidance probabilities for individual regions. Even though this lemma is a direct conclusion from the graphical construction, we state it in a separate section due to its importance in our further argument.

Lemma 4.1 Assume that $U_{1}, U_{2}, \ldots, U_{k}, k \geq 1$ are disjoint bounded regions in $\mathbb{R}^{2}$ such that $\min _{i \neq j} \operatorname{dist}\left(U_{i}, U_{j}\right)>\Delta \gg \log \left[k \sup _{i=1}^{k} \operatorname{diam}\left(U_{i}\right)\right]$. Then, for some $C>0$ we have

$$
\begin{gathered}
\mathbb{P}\left(\mathcal{A}^{[\beta]} \cap \bigcup_{j=1}^{k} U_{j}=\emptyset\right)= \\
\left(1+O\left((\log k) \mathrm{e}^{-C \Delta} \sum_{i=1}^{k} \operatorname{Area}\left(U_{i} \oplus \mathbb{B}_{2}(1)\right)\right)\right) \prod_{j=1}^{k} \mathbb{P}\left(\mathcal{A}^{[\beta]} \cap U_{j}=\emptyset\right) .
\end{gathered}
$$

Proof The exponential decay of ancestor clan sizes in the graphical construction (1.7) yields

$$
\mathbb{P}\left(\mathcal{E}_{i}^{c}\right)=O\left(\operatorname{Area}\left(U_{i} \oplus \mathbb{B}_{2}(1)\right) \exp (-C \Delta)\right), i=1, \ldots, k
$$


with

$$
\mathcal{E}_{i}:=\left\{\operatorname{An}_{0}\left(U_{i}\right) \subseteq U_{i} \oplus \mathbb{B}_{2}(\Delta / 2)\right\}
$$

Write $\mathcal{I}_{j}, j=1, \ldots, k$ for the event

$$
\mathcal{I}_{j}:=\left\{\mathcal{A}^{[\beta]} \cap U_{j}=\emptyset\right\}
$$

and use the canonical coupling of the graphical construction for $\mathcal{A}^{[\beta]}$ with the conditional graphical construction for the field $\mathcal{A}_{\mathbb{R}^{2}:\left[\bigcup_{i=1}^{\lfloor k / 2\rfloor} U_{i}\right]}^{[\beta]}$ as provided in Section 1.2 to conclude that

$$
\left|\mathbb{P}\left(\bigcap_{i=\lfloor k / 2\rfloor+1}^{k} \mathcal{I}_{i} \mid \bigcap_{i=1}^{\lfloor k / 2\rfloor} \mathcal{I}_{i}\right)-\mathbb{P}\left(\bigcap_{i=\lfloor k / 2\rfloor+1}^{k} \mathcal{I}_{i}\right)\right| \leq \mathbb{P}\left(\bigcup_{i=1}^{k} \mathcal{E}_{i}^{c}\right)
$$

Combining (4.32) with (4.33) leads to

$$
\mathbb{P}\left(\bigcap_{i=1}^{k} \mathcal{I}_{i}\right)=\left(1+O\left(\sum_{i=1}^{k} \operatorname{Area}\left(U_{i} \oplus \mathbb{B}_{2}(1)\right) \mathrm{e}^{-C \Delta}\right)\right) \mathbb{P}\left(\bigcap_{i=1}^{\lfloor k / 2\rfloor} \mathcal{I}_{i}\right) \mathbb{P}\left(\bigcap_{i=\lfloor k / 2\rfloor+1}^{k} \mathcal{I}_{i}\right)
$$

The assertion of the lemma follows now by recursive application of (4.34).

\section{Existence and properties of surface tension}

This section deals with the existence of the limit (1.15) defining the surface tension functional specific for our model. The argument below relies on a number of technical properties of the quantity $T_{(\cdot)}^{[\beta]}[\cdot \leftrightarrow \cdot]$ and is split into several subsections. Our main tool here is the random walk representation of surface tension, stated in Lemma 5.1, and our main effort is concentrated on establishing the crucial finite volume approximation Lemma 5.2. As everywhere in this paper, the results below are valid for $\beta$ large enough.

\subsection{Optimising and freezing initial segments}

It will be convenient for our further purposes to switch between several alternative but asymptotically equivalent variants and representations of the surface tension. In this subsection we argue that modifying and freezing the directions of the initial segments of the polygonal path in the original definition (1.13) of the functional $T_{(\delta)}^{[\beta]}[x \leftrightarrow y]$ does not alter its asymptotic behaviour for large $\operatorname{dist}(x, y)$. To this end we consider a version $\hat{T}_{(\delta)}^{[\beta]}[x \leftrightarrow y]$ of the quantity $T_{(\delta)}^{[\beta]}[x \leftrightarrow y]$, which arises as the supremum of the integrals as in (1.13), 
but in which the initial point of the first segment is now allowed in the whole $\mathbb{B}_{2}(x, \delta)$ rather than just on $\partial \mathbb{B}_{2}(x, \delta)$, the endpoint of the last segment is allowed in the whole $\mathbb{B}_{2}(y, \delta)$ rather than just on $\partial \mathbb{B}_{2}(y, \delta)$, and the directions of both segments are fixed so that the integration is carried out over the remaining segments only. It is easily checked that, provided the distance between $x$ and $y$ is large enough,

$$
C^{-1} T_{(\delta)}^{[\beta]}[x \leftrightarrow y] \leq \hat{T}_{(\delta)}^{[\beta]}[x \leftrightarrow y] \leq C T_{(\delta)}^{[\beta]}[x \leftrightarrow y]
$$

for some $C=C(\beta, \delta)>1$ independent of $D, x, y$. Indeed, the impact of taking the first and last segments fixed in the optimal way rather than integrating over them is easily seen to be only confined to close neighbourhoods of the initial point and the endpoint of the path, and can be compensated at a constant probability cost by appropriately adjusting a small number of initial and final segments. We also consider finite volume versions $T_{(\delta ; D)}^{[\beta]}$ and $\hat{T}_{(\delta ; D)}^{[\beta]}$ of $T_{(\delta)}^{[\beta]}$ and $\hat{T}_{(\delta)}^{[\beta]}$, putting in analogy with (1.13)

$$
T_{(\delta ; D)}^{[\beta]}[x \leftrightarrow y]:=\int_{\mathcal{C}^{x \leftrightarrow y ; \delta}} \mathbb{P}\left(\mathcal{A}^{[\beta]} \cap \theta=\emptyset\right)\left[\Theta_{D}^{x \leftrightarrow y ; \delta}\right]^{[\beta]}(d \theta)
$$

and defining $\hat{T}_{(\delta ; D)}^{[\beta]}$ in the same way as $\hat{T}_{(\delta)}^{[\beta]}$ with the additional requirement that the whole path be contained in $D$. If the domain $D$ contains neighbourhoods of $x$ and $y$ (say, $\mathbb{B}_{2}(x, 2 \delta) \subseteq D$ and $\mathbb{B}_{2}(y, 2 \delta) \subseteq D$ ), a relation analogous to (5.35) is easily verified to hold for $x$ and $y$ far enough

$$
C^{-1} T_{(\delta ; D)}^{[\beta]}[x \leftrightarrow y] \leq \hat{T}_{(\delta ; D)}^{[\beta]}[x \leftrightarrow y] \leq C T_{(\delta ; D)}^{[\beta]}[x \leftrightarrow y]
$$

with some $C:=C(\beta, \delta)>1$ independent of $D, x, y$.

We close this subsection with one more quantity, to be of use in the sequel, for which a relation analogous to (5.35) and (5.36) is valid. Write

$$
\vartheta_{(\delta)}^{[\beta]}[x \leftrightarrow y]=\int_{\mathcal{C}^{x \leftrightarrow y ; \delta}}\left[\Theta^{x \leftrightarrow y ; \delta}\right]^{[\beta]}(d \theta)=\left[\Theta^{x \leftrightarrow y ; \delta}\right]^{[\beta]}\left(\mathcal{C}^{x \leftrightarrow y ; \delta}\right)
$$

and, as in the definition of $\hat{T}_{(\cdot)}^{[\cdot]}[\cdot \leftrightarrow \cdot]$, let $\hat{\vartheta}_{(\delta)}^{[\beta]}[x \leftrightarrow y]$ be the supremum of integrals as in (5.37), but with the initial point of the first segment now allowed in the whole $\mathbb{B}_{2}(x, \delta)$ rather than just on $\partial \mathbb{B}_{2}(x, \delta)$, the endpoint of the last segment allowed in the whole $\mathbb{B}_{2}(y, \delta)$ rather than just on $\partial \mathbb{B}_{2}(y, \delta)$, and the directions of both segments fixed so that the integration is carried out over the remaining segments only. Clearly, in full analogy to (5.35), we have with $\operatorname{dist}(x, y)$ large enough

$$
C^{-1} \vartheta_{(\delta)}^{[\beta]}[x \leftrightarrow y] \leq \hat{\vartheta}_{(\delta)}^{[\beta]}[x \leftrightarrow y] \leq C \vartheta_{(\delta)}^{[\beta]}[x \leftrightarrow y]
$$


for some $C=C(\beta, \delta)>1$ independent of $D, x, y$.

\subsection{Random walk representation}

The quantity $T_{(\delta)}^{[\beta]}[x \leftrightarrow y]$ admits a particularly useful interpretation in terms of a killed continuum random walk in environment with random obstacles. To see it consider a continuous-time random walk $Z_{t ; \mathbb{B}_{2}(x, \delta)}:=Z_{t}$ in $\mathbb{R}^{2}$ independent of $\mathcal{A}^{[\beta]}$ and governed by the following dynamics

- between critical events specified below move in a constant direction with speed 1 ,

- with intensity given by 4 times the covered length element update the movement direction, choosing the angle $\phi \in(0,2 \pi)$ between the old and new direction according to the density $|\sin (\phi)| / 4$.

The starting point and the initial velocity direction for $Z_{t}$ are chosen by taking a straight line $l$ crossing $\mathbb{B}_{2}(x, \delta)$ according to the measure $\mu(\cdot) / \mu\left(\left\{l \mid l \cap \mathbb{B}_{2}(x, \delta) \neq \emptyset\right\}\right)$. The starting point of $Z_{t}$ is now taken to be one of the intersection points of $l$ with $\partial \mathbb{B}_{2}(x, \delta)$, each picked with probability $1 / 2$, while the initial velocity vector lies on $l$ pointing outwards $\mathbb{B}_{2}(x, \delta)$. Let $\tilde{Z}_{t}=\tilde{Z}_{t ; \mathbb{B}_{2}(x, \delta)}$ be the random walk $Z_{t}$ killed whenever hitting its past trajectory. A crucial observation is that the probability element of the walk $Z_{t}$ containing a given polygonal path $\theta \in \mathcal{C}^{x \leftrightarrow y ; \delta}$ as its initial subpath is exactly

$$
\frac{1}{2 \mu\left(\left\{l \mid l \cap \mathbb{B}_{2}(x, \delta) \neq \emptyset\right\}\right)} \exp (-4 \text { length }(\theta)) \prod_{i=1}^{k} d \mu\left(l\left[e_{k}\right]\right),
$$

where $e_{1}, \ldots, e_{k}$ are the segments of $\theta$ while $l\left[e_{i}\right]$ stands for the straight line determined by $e_{i}$. Indeed, the prefactor $\left[2 \mu\left(\left\{l \mid l \cap \mathbb{B}_{2}(x, \delta) \neq \emptyset\right\}\right)\right]^{-1}$ comes from the choice of the initial segment of $Z_{t}$ [the line on which it lies and one out of two equiprobable directions, whence the extra $2^{-1}$ ] while for the remaining segments we use the fact that, for any given straight line $l_{0}$, we have $\mu\left(\left\{l \mid l \cap l_{0} \in d \ell, \angle\left(l, l_{0}\right) \in d \phi\right\}\right)=|\sin \phi| d \ell d \phi$ with $d \ell$ standing for the length element on $l_{0}$ and with $\angle\left(l_{0}, l\right)$ denoting the angle between $l$ and $l_{0}$, see Proposition 3.1 in [2] as well as the argument justifying the dynamic representation of the Arak in Section 4 ibidem and the proof of Lemma 1 in Schreiber [15]. Note that the direction update intensity for $Z_{t}$ was set to 4 to cancel out with the normalising constant $\int_{0}^{2 \pi}|\sin \phi| d \phi=4$ in the density $|\sin \phi| / 4$ for the new angle choice. Clearly, the formula (5.39) is also valid for $Z_{t}$ replaced by $\tilde{Z}_{t}$ since the paths in $\mathcal{C}^{x \leftrightarrow y ; \delta}$ are by definition self-avoiding. Thus, taking 
into account that, by standard integral geometry, $\mu\left(\left\{l \mid l \cap \mathbb{B}_{2}(x, \delta) \neq \emptyset\right\}\right)=2 \pi \delta$ and recalling (1.11) and (1.12) we rewrite (5.39) as $\frac{1}{4 \pi \delta}\left[\Theta^{x \leftrightarrow y ; \delta}\right]^{[2]}(d \theta)$. Consequently, recalling the definition of $\mathcal{C}^{x \leftrightarrow y ; \delta}$ and using (5.39) we come to

Lemma 5.1 For each $C \subseteq \mathcal{C}^{x \leftrightarrow y ; \delta}$ the following representation formula is valid for the value of $\left[\Theta^{x \leftrightarrow y ; \delta}\right]^{[2]}(C)$

$$
\left[\Theta^{x \leftrightarrow y ; \delta}\right]^{[2]}(C)=4 \pi \delta \mathbb{E} \operatorname{card}\left\{\tilde{t}>0 \mid \tilde{Z}_{\tilde{t}} \in\left(\tilde{Z}_{t}\right)_{t \geq 0} \cap_{\text {in }} \partial \mathbb{B}_{2}(y, \delta), \tilde{Z}_{[0, \tilde{t}]} \in C\right\}
$$

where $\left(\tilde{Z}_{t}\right)_{t \geq 0} \cap_{\text {in }} \partial \mathbb{B}_{2}(y, \delta)$ stands for the collection of entry points of $\tilde{Z}_{t}$ into $\mathbb{B}_{2}(y, \delta)$, with exit points not taken into account.

A simple yet useful conclusion of Lemma 5.1 is that, denoting by $\tilde{Z}_{t}^{[\beta]}=\tilde{Z}_{t ; \mathbb{B}_{2}(x, \delta)}^{[\beta]}$ the random walk $Z_{t}$ killed at rate $\beta$ and, in addition, killed whenever hitting its past trajectory, we have for $\beta \geq 2$

$$
\left[\Theta^{x \leftrightarrow y ; \delta}\right]^{[\beta]}(C)=4 \pi \delta \mathbb{E} \operatorname{card}\left\{\tilde{t}>0 \mid \tilde{Z}_{\tilde{t}}^{[\beta-2]} \in\left(\tilde{Z}_{t}^{[\beta-2]}\right)_{t \geq 0} \cap_{\text {in }} \partial \mathbb{B}_{2}(y, \delta), \tilde{Z}_{[0, \tilde{t}]}^{[\beta-2]} \in C\right\}
$$

Consequently, writing now $\hat{Z}_{t}^{[\beta]}=\hat{Z}_{t ; \mathbb{B}_{2}(x, \delta)}^{[\beta]}$ for the random walk $Z_{t}$ killed at rate $\beta$ and, in addition, killed whenever hitting its past trajectory or a contour of $\mathcal{A}^{[\beta]}$, in view of Lemma 5.1 and (5.40) the definition (1.13) yields

$$
T_{(\delta)}^{[\beta]}[x \leftrightarrow y]=4 \pi \delta \mathbb{E} \operatorname{card}\left[\left(\hat{Z}_{t}^{[\beta-2]}\right)_{t \geq 0} \cap_{\text {in }} \partial \mathbb{B}_{2}(y, \delta)\right]
$$

A similar representation can be provided for $T_{(\delta ; D)}^{[\beta]}$, by additionally killing the random walk whenever it hits $\partial D$. A corresponding representation for $\hat{T}_{(\delta)}^{[\beta]}$ and $\hat{T}_{(\delta ; D)}^{[\beta]}$ can also be given, yet we omit it because it is unessential for our further purposes and involves certain technicalities due the fixed last segment.

\subsection{Finite volume approximations}

The following lemma shows that $T_{(\delta)}^{[\beta]}[x \leftrightarrow y]$ is well approximated by $T_{(\delta ; D)}^{[\beta]}[x \leftrightarrow y]$ for sufficiently large domains $D$. We write $\Pi(x \leftrightarrow y ; \delta)$ for the square of sidelength $2 \delta+$ $\operatorname{dist}(x, y)$ with one pair of its sides parallel and equidistant to $[x, y]$ and with the remaining two sides at the distance $\delta$ from $x$ and $y$ respectively, perpendicular to $[x, y]$. 
Lemma 5.2 For each sufficiently large $\beta>2$ there exists a constant $C=C(\beta, \delta)>0$ such that

$$
C^{-1} T_{(\delta)}^{[\beta]}[x \leftrightarrow y] \leq T_{(\delta ; \Pi(x \leftrightarrow y ; \delta))}^{[\beta]}[x \leftrightarrow y] \leq T_{(\delta)}^{[\beta]}[x \leftrightarrow y]
$$

whenever dist $(x, y)$ is large enough.

Proof The relation $T_{(\delta ; \Pi(x \leftrightarrow y ; \delta))}^{[\beta]}[x \leftrightarrow y] \leq T_{(\delta)}^{[\beta]}[x \leftrightarrow y]$ is obvious and only the remaining inequality $T_{(\delta)}^{[\beta]}[x \leftrightarrow y] \leq C T_{(\delta ; \Pi(x \leftrightarrow y ; \delta))}^{[\beta]}[x \leftrightarrow y]$ requires verification. In view of the random walk representation (5.41) it will follow as soon as we show that

$$
P_{(\delta ; \Pi(x \leftrightarrow y ; \delta))}^{[\beta]}[x \leftrightarrow y] \geq C^{-1} P_{(\delta)}^{[\beta]}[x \leftrightarrow y]
$$

for some $C>0$, where

$$
P_{(\delta)}^{[\beta]}[x \leftrightarrow y]:=\mathbb{P}\left(\left(\hat{Z}_{t}^{[\beta-2]}\right)_{t \geq 0} \text { reaches } \partial \mathbb{B}_{2}(y, \delta)\right)
$$

and

$$
P_{(\delta ; D)}^{[\beta]}[x \leftrightarrow y]:=\mathbb{P}\left(\left(\hat{Z}_{t}^{[\beta-2]}\right)_{t \geq 0} \text { reaches } \partial \mathbb{B}_{2}(y, \delta) \text { before hitting } \partial D\right) .
$$

Indeed, it is easily argued that upon hitting $\partial \mathbb{B}_{2}(y, \delta)$ once, the random walk $\hat{Z}_{t}^{[\beta-2]}$ is unlikely to hit it too many more times and, consequently, the expectation on the right-hand side of (5.41) is bounded above and below by some constant multiplicities of the probability on the right-hand side of (5.42), the same observation holds for the corresponding representation of the finite-volume quantity $T_{(\delta ; \Pi(x \leftrightarrow y ; \delta))}^{[\beta]}[x \leftrightarrow y]$. We omit the tedious technical details of this conceptually simple argument.

The proof of (5.42) splits into two parts. First, denoting by $R_{1}(x \leftrightarrow y ; \delta)$ the infinite strip between the lines determined by the sides of $\Pi(x \leftrightarrow y ; \delta)$ perpendicular to $[x, y]$, we show that

$$
P_{(\delta)}^{[\beta]}[x \leftrightarrow y] \leq C P_{\left(\delta ; R_{1}(x \leftrightarrow y ; \delta)\right)}^{[\beta]}[x \leftrightarrow y]
$$

for some $C>0$. Below it will be convenient to use the name $x$-line (resp. $y$-line) for the boundary line (side) of $R_{1}(x \leftrightarrow y ; \delta)$ at the distance $\delta$ from $x$ (resp. $y$ ), perpendicular to $[x, y]$. Next, writing $R_{2}(x \leftrightarrow y ; \delta)$ for the infinite strip contained between the lines determined by the sides of $\Pi(x \leftrightarrow y ; \delta)$ parallel to $[x, y]$, we show that

$$
P_{(\delta)}^{[\beta]}[x \leftrightarrow y] \leq P_{\left(\delta ; R_{2}(x \leftrightarrow y ; \delta)\right)}^{[[]}[x \leftrightarrow y](1+o(1)) .
$$


as $\operatorname{dist}(x, y) \rightarrow \infty$. Write

$$
\begin{gathered}
P_{(\delta ; \Pi(x \leftrightarrow y ; \delta))}^{[\beta]}[x \leftrightarrow y] \geq P_{(\delta)}^{[\beta]}[x \leftrightarrow y]-\left(P_{(\delta)}^{[\beta]}[x \leftrightarrow y]-P_{\left(\delta ; R_{1}(x \leftrightarrow y ; \delta)\right)}^{[\beta]}[x \leftrightarrow y]\right)- \\
\left(P_{(\delta)}^{[\beta]}[x \leftrightarrow y]-P_{\left(\delta ; R_{2}(x \leftrightarrow y ; \delta)\right)}^{[\beta]}[x \leftrightarrow y]\right)=P_{\left(\delta ; R_{1}(x \leftrightarrow y ; \delta)\right)}^{[\beta]}[x \leftrightarrow y]+P_{\left(\delta ; R_{2}(x \leftrightarrow y ; \delta)\right)}^{[\beta]}[x \leftrightarrow y]- \\
P_{(\delta)}^{[\beta]}[x \leftrightarrow y] .
\end{gathered}
$$

Combining this with (5.43) and (5.44) yields (5.42) as required for completing the proof of the lemma.

To proceed with the verification of (5.43), on the event that the random walk $\hat{Z}_{t}^{[\beta-2]}$ reaches $\partial \mathbb{B}_{2}(y, \delta)$ before being killed we decompose its trajectory into three subpaths

- $\zeta_{x \leftrightarrow y ; \delta}:=\left(\hat{Z}_{t}^{[\beta-2]}\right)_{\left[\tau_{x}, \tau_{y}\right]}$, where $\tau_{y}$ is the first time $\hat{Z}_{t}^{[\beta-2]}$ hits the $y$-line while $\tau_{x}$ is the last time $\hat{Z}_{t}^{[\beta-2]}$ hits the $x$-line before $\tau_{y}$.

- $\zeta_{x}:=\left(\hat{Z}_{t}^{[\beta-2]}\right)_{\left[0, \tau_{x}\right]}$,

- $\zeta_{y}:=\left(\hat{Z}_{t}^{[\beta-2]}\right)_{t \geq \tau_{y}}$,

with the additional convention that $\tau_{x}:=0$ if $\hat{Z}_{t}^{[\beta-2]}$ does not reach the $x$-line and $\tau_{y}:=+\infty$ if $\hat{Z}_{t}^{[\beta-2]}$ does not reach the $y$-line (we set respectively $\zeta_{x}:=\emptyset$ and $\zeta_{y}:=\emptyset$ in these cases). On the $x$-line we construct a double sequence $\left(x_{i}\right)_{i \in \mathbb{Z}}$ of points with $x_{i+k}$ lying at the distance $|k| \delta$ from $x_{i}$ (say above for $k>0$, below for $k<0$ ) and with $x_{0}$ coinciding with the intersection point of the $x$-line and the line extending $[x, y]$. The sequence $\left(y_{i}\right)_{i \in \mathbb{Z}}$ on the $y$-line is constructed in the same way and ordered in the same direction as $\left(x_{i}\right)$. Let $\hat{x}$ denote the point in $\left(x_{i}\right)_{i \in \mathbb{Z}}$ which lies the closest to $\hat{Z}_{\tau_{x}}^{[\beta]}$ if $\tau_{x}>0$ and $\hat{x}:=x$ otherwise. Likewise, let $\hat{y}$ be the point in $\left(y_{i}\right)_{i \in \mathbb{Z}}$ lying the closest to $\hat{Z}_{\tau_{y}}^{[\beta-2]}$ if $\tau_{y}<+\infty$ and $\hat{y}:=y$ otherwise. With this notation it is easily seen that

$$
P_{(\delta)}^{[\beta]}[x \leftrightarrow y] \leq \sum_{\hat{x} \in\{x\} \cup\left\{x_{i}, i \in \mathbb{Z}\right\}} \sum_{\hat{y} \in\{y\} \cup\left\{y_{j}, j \in \mathbb{Z}\right\}} P_{\left(\delta ; R_{1}(x \leftrightarrow y ; \delta)\right)}^{[\beta]}[\hat{x} \leftrightarrow \hat{y}] Q_{(\delta)}^{[\beta]}[x \leftrightarrow \hat{x} ; \hat{y} \leftrightarrow y]
$$

where $Q_{(\delta)}^{[\beta]}[x \leftrightarrow \hat{x} ; \hat{y} \leftrightarrow y]$ stands for the supremum over the possible realisations of $\zeta_{x \leftrightarrow y}$ connecting $\mathbb{B}_{2}(\hat{x}, \delta)$ with $\mathbb{B}_{2}(\hat{y}, \delta)$ of the conditional probability, given $\zeta_{x \leftrightarrow y}$, that the random walk $\hat{Z}_{t}^{[\beta-2]}$ connects $\mathbb{B}_{2}(x, \delta)$ to $\mathbb{B}_{2}(\hat{x}, \delta)$ and $\mathbb{B}_{2}(\hat{y}, \delta)$ to $\mathbb{B}_{2}(y, \delta)$. Since $\hat{Z}_{t}^{[\beta-2]}$ is killed with 
the constant rate $\beta-2>0$, for arbitrarily small $\epsilon$ we can find $\lambda=\lambda(\epsilon)$ such that, uniformly over $x, y$ with $\operatorname{dist}(x, y)$ large enough,

$$
\sum_{x_{i}, \operatorname{dist}\left(x_{i}, x\right)>\lambda} \sum_{y_{j}} Q_{(\delta)}^{[\beta]}\left[x \leftrightarrow x_{i} ; y_{j} \leftrightarrow y\right]+\sum_{x_{i}} \sum_{y_{j}, \operatorname{dist}\left(y_{j}, y\right)>\lambda} Q_{(\delta)}^{[\beta]}\left[x \leftrightarrow x_{i} ; y_{j} \leftrightarrow y\right] \leq \epsilon .
$$

Putting (5.45) and (5.46) together yields

$$
P_{(\delta)}^{[\beta]}[x \leftrightarrow y] \leq \sum_{x_{i}, \operatorname{dist}\left(x_{i}, x\right) \leq \lambda ; y_{j}, \operatorname{dist}\left(y_{j}, y\right) \leq \lambda} P_{\left(\delta ; R_{1}(x \leftrightarrow y ; \delta)\right)}^{[\beta]}\left[x_{i} \leftrightarrow y_{j}\right]+\epsilon \sup _{x_{i}, y_{j}} P_{\left(\delta ; R_{1}(x \leftrightarrow y ; \delta)\right)}^{[\beta]}\left[x_{i} \leftrightarrow y_{j}\right] .
$$

For dist $(x, y)$ large enough the double sum in (5.47) can be bounded above by some constant $C[\lambda]$ times $P_{\left(\delta ; R_{1}(x \leftrightarrow y ; \delta)\right)}^{[\beta]}[x \leftrightarrow y]$ because each path of $\hat{Z}_{t}^{[\beta-2]}$ connecting $\partial \mathbb{B}_{2}\left(x_{i}, \delta\right)$ to $\partial \mathbb{B}_{2}\left(y_{j}, \delta\right)$ in $R_{1}(x \leftrightarrow y ; \delta)$ with $\operatorname{dist}\left(x, x_{i}\right) \leq \lambda$ and $\operatorname{dist}\left(y, y_{j}\right) \leq \lambda$ can be modified into a path connecting $\partial \mathbb{B}_{2}(x, \delta)$ to $\partial \mathbb{B}_{2}(y, \delta)$ in $R_{1}(x \leftrightarrow y ; \delta)$ by an appropriate surgery between $x$ and $x_{i}$ and between $y$ and $y_{j}$ at a probability cost depending only on $\lambda$. It seems natural to expect that the $\operatorname{supremum}_{\sup _{x_{i}, y_{j}}} P_{\left(\delta ; R_{1}(x \leftrightarrow y ; \delta)\right)}^{[\beta]}\left[x_{i} \leftrightarrow y_{j}\right]$ admits an upper bound very close to $P_{(\delta)}^{[\beta]}[x \leftrightarrow y]$, because $\operatorname{dist}\left(x_{i}, y_{j}\right)>\operatorname{dist}(x, y)$ for all $x_{i}, y_{j}$. While we are not able to establish such a bound, we easily show that there exists a positive constant $C^{\prime}$ with

$$
\sup _{x_{i}, y_{j}} P_{\left(\delta ; R_{1}(x \leftrightarrow y ; \delta)\right)}^{[\beta]}\left[x_{i} \leftrightarrow y_{j}\right] \leq C^{\prime} P_{(\delta)}^{[\beta]}[x \leftrightarrow y]
$$

uniformly in $x, y$ with dist $(x, y)$ large enough. Indeed, this is done much along the same lines as in the considerations leading to (5.45) and (5.47), so we only sketch the argument omitting technical details. We split each path of the random walk $\hat{Z}_{t ; \mathbb{B}_{2}\left(x_{i}, \delta\right)}^{[\beta-2]}$ connecting $\partial \mathbb{B}_{2}\left(x_{i}, \delta\right)$ to $\partial \mathbb{B}_{2}\left(y_{j}, \delta\right)$ into two subpaths: the initial subpath $\zeta_{1}$ connecting $\partial \mathbb{B}_{2}\left(x_{i}, \delta\right)$ to some $\partial \mathbb{B}_{2}(z, \delta), z \in \delta \mathbb{Z}^{2}$ with $\left|\operatorname{dist}\left(x_{i}, z\right)-\operatorname{dist}(x, y)\right| \leq \delta$ (in fact, $z$ can be chosen as the $\delta \mathbb{Z}^{2}$-lattice point closest to the point where the random walk $\hat{Z}_{t ; \mathbb{B}_{2}\left(x_{i}, \delta\right)}^{[\beta-2]}$ first gets at the distance $\operatorname{dist}(x, y)$ away from $\left.x_{i}\right)$ and the remaining subpath $\zeta_{2}$. Integrating over $\zeta_{1}$ for fixed $z$ yields a value bounded above by a constant multiplicity of $P_{(\delta)}^{[\beta]}[x \leftrightarrow y]$ with this prefactor (arbitrarily close to 1 for $\delta$ small enough) due to the fact that $\operatorname{dist}\left(x_{i}, z\right)$ differs slightly from $\operatorname{dist}(x, y)$. Integrating over $\zeta_{2}$ conditioned on $\zeta_{1}$ and summing over $z$ yields only a constant prefactor - the sum of integrals converges due to the constant killing rate $\beta-2>0$ along $\zeta_{2}$. This proves (5.48). Combining now (5.47) with (5.48) and with the discussion directly following (5.47) we obtain

$$
P_{(\delta)}^{[\beta]}[x \leftrightarrow y] \leq C[\lambda] P_{\left(\delta ; R_{1}(x \leftrightarrow y ; \delta)\right)}^{[\beta]}[x \leftrightarrow y]+\epsilon C^{\prime} P^{[\beta]}[x \leftrightarrow y] .
$$


Choosing $\epsilon$ small enough so that $\epsilon C^{\prime}<1$ (recall that $C^{\prime}$ does not depend on $\lambda$ ) completes the proof of (5.43).

To establish $(5.44)$ we denote by $\overrightarrow{v_{x y}}$ the unit vector pointing from $x$ to $y$, i.e. $\overrightarrow{v_{x y}}:=$ $(y-x) / \operatorname{dist}(x, y)$, and for small $\eta>0$ we consider the event $\mathcal{E}_{(\delta ; \eta)}[x \leftrightarrow y]$ that

- The random walk $\left(\hat{Z}_{t}^{[\beta-2]}\right)_{t \geq 0}$ reaches $\partial \mathbb{B}_{2}(y, \delta)$,

- The scalar product of $\overrightarrow{v_{x y}}$ and the current velocity vector of $\hat{Z}_{t}^{[\beta-2]}$ is in $[1-\eta, 1]$ for all time moments $t \geq 0$ before $\partial \mathbb{B}_{2}(y, \delta)$ is reached.

Observe that on the event $\mathcal{E}_{(\delta ; \eta)}^{[\beta]}[x \leftrightarrow y]$ the total length of the path of $\hat{Z}_{t}^{[\beta-2]}$ connecting $\partial \mathbb{B}_{2}(x, \delta)$ to $\partial \mathbb{B}_{2}(y, \delta)$ cannot exceed $[\operatorname{dist}(x, y)+2 \delta] /[1-\eta]$. Since $\mathcal{A}^{[\beta]}$ is stochastically dominated by the Poisson contour process $\mathcal{P}_{\Theta[\beta]}$, as follows by the graphical construction of Section 1.2, we conclude that there exists $\kappa>0$ such that for all $\beta$ large enough

$$
\mathbb{P}\left(\mathcal{E}_{(\delta ; \eta)}^{[\beta]}[x \leftrightarrow y]\right) \geq \exp \left(-\left[\frac{\beta-2}{1-\eta}+\kappa\right](\operatorname{dist}(x, y)+2 \delta)\right) .
$$

Indeed, to see it we:

- Split the strip $R_{1}(x \leftrightarrow y ; \delta)$ with equidistant straight lines perpendicular to $[x, y]$ into $\Theta(\operatorname{dist}(x, y))$ equal-sized strips.

- Construct a path of the random walk $\tilde{Z}$ connecting $\partial \mathbb{B}_{2}(x, \delta)$ to $\partial \mathbb{B}_{2}(y, \delta)$ and such that the scalar product of $\overrightarrow{x y}$ and the current velocity vector of $\tilde{Z}_{t}$ falls into $[1-\eta, 1]$ for all time moments before $\partial \mathbb{B}_{2}(y, \delta)$ is reached. This is done by constructing and patching together subpaths of $\tilde{Z}$ crossing individual strips, at a constant probability cost per strip.

- Use the stochastic domination of $\mathcal{A}^{[\beta]}$ by $\mathcal{P}_{\Theta[\beta]}$ to conclude that the probability that the so constructed path of $\tilde{Z}_{t}$ avoids $\mathcal{A}^{[\beta]}$ is bounded below by $\exp (-\Theta(\operatorname{dist}(x, y)))$.

- Check for survival of the so constructed path under $\beta-2$-killing, which yields a probability prefactor bounded below by $\exp \left(-\frac{\beta-2}{1-\eta}[\operatorname{dist}(x, y)+2 \delta]\right)$.

By the definition of $\mathcal{E}_{(\delta ; \eta)}^{[\beta]}$, for $\operatorname{dist}(x, y)$ large enough this procedure allows us to bound below the probability of this event by $\exp \left(-\left(\frac{\beta-2}{1-\eta}+\kappa\right)[\operatorname{dist}(x, y)+2 \delta]\right)$ for some $\kappa>0$, as required. Since $\mathcal{P}_{\Theta[\beta]}$ stochastically dominates $\mathcal{P}_{\Theta^{\left[\beta^{\prime}\right]}}$ for $\beta^{\prime}>\beta$, this technique works uniformly in $\beta$ large enough. We omit tedious technical details of this standard argument. To proceed, define the event $\mathcal{R}_{(\delta)}^{[\beta]}[x \leftrightarrow y]$ that 
- The random walk $\left(\hat{Z}_{t}^{[\beta-2]}\right)_{t \geq 0}$ reaches $\partial \mathbb{B}_{2}(y, \delta)$,

- The random walk $\left(\hat{Z}_{t}^{[\beta-2]}\right)_{t \geq 0}$ hits $\partial R_{2}(x \leftrightarrow y ; \delta)$ before reaching $\partial \mathbb{B}_{2}(y, \delta)$,

and observe that on $\mathcal{R}_{(\delta)}^{[\beta]}[x \leftrightarrow y]$ the length of the path connecting $\partial \mathbb{B}_{2}(x, \delta)$ to $\partial \mathbb{B}_{2}(y, \delta)$ has to exceed $\sqrt{5 / 4} \operatorname{dist}(x, y)-2 \delta$ and, hence,

$$
\mathbb{P}\left(\mathcal{R}_{(\delta)}^{[\beta]}[x \leftrightarrow y]\right) \leq \exp (-(\beta-2)[\sqrt{5 / 4} \operatorname{dist}(x, y)-2 \delta]) .
$$

Noting that $\mathbb{P}\left(\mathcal{E}_{(\delta ; \eta)}^{[\beta]}[x \leftrightarrow y]\right) \leq P_{\left(\delta ; R_{2}(x \leftrightarrow y ; \delta)\right)}^{[\beta]}[x \leftrightarrow y]$ for sufficiently small $\eta$ and putting (5.50) together with (5.51) we see that, for $\beta$ large enough,

$$
P_{(\delta)}^{[\beta]}[x \leftrightarrow y]-P_{\left(\delta ; R_{2}(x \leftrightarrow y ; \delta)\right)}^{[\beta]}[x \leftrightarrow y]=\mathbb{P}\left(\mathcal{R}_{(\delta)}^{[\beta]}[x \leftrightarrow y]\right)=o\left(P_{\left(\delta ; R_{2}(x \leftrightarrow y ; \delta)\right)}^{[\beta]}[x \leftrightarrow y]\right) .
$$

This yields (5.44) and hence the required relation (5.42). The proof of Lemma 5.2 is complete.

\subsection{Existence and finiteness of surface tension}

In this subsection we use the preceding results of this section to show that

Lemma 5.3 The limit defining the surface tension functional $\tau^{[\beta]}$ in (1.15) exists, is finite and strictly positive.

Proof The main work has already been done in Lemma 5.2. In view of the relation $\sup _{\lambda>2 \delta} \tau_{\lambda}^{[\beta]}<\infty$ as easily deduced from (5.50), the required existence of the limit in (1.15) will follow by a standard almost-subadditivity argument once we establish the following auxiliary lemma.

Lemma 5.4 For $D\left[\lambda_{1}, \lambda_{2}\right]:=\left(\log \left[\lambda_{1}+\lambda_{2}\right]\right)^{2}$ and $\lambda_{1}, \lambda_{2}$ large enough we have

$$
\left.\left(\lambda_{1}+\lambda_{2}+D\left[\lambda_{1}, \lambda_{2}\right]\right) \tau_{\lambda_{1}+\lambda_{2}+D\left[\lambda_{1}, \lambda_{2}\right]}^{[\beta]} \leq \lambda_{1} \tau_{\lambda_{1}}^{[\beta]}+\lambda_{2} \tau_{\lambda_{2}}^{[\beta]}+O\left(D\left[\lambda_{1}, \lambda_{2}\right]\right)\right) .
$$

Proof of Lemma 5.4 For fixed $\delta>0$ consider disjoint squares $\Pi_{1}:=\Pi\left(0 \leftrightarrow \lambda_{1} \mathbf{e}_{x} ; \delta\right)$ and $\Pi_{2}:=\Pi\left(\left(\lambda_{1}+D\left[\lambda_{1}, \lambda_{2}\right]\right) \mathbf{e}_{x} \leftrightarrow\left(\lambda_{1}+\lambda_{2}+D\left[\lambda_{1}, \lambda_{2}\right]\right) \mathbf{e}_{x} ; \delta\right)$, separated by a moat of width $D\left[\lambda_{1}, \lambda_{2}\right]$, and observe that, since $D\left[\lambda_{1}, \lambda_{2}\right] \gg \log \left[\lambda_{1}+\lambda_{2}\right]$, by the decoupling Lemma 4.1 applied to $U_{1}:=\Pi_{1} \cap \theta, U_{2}:=\Pi_{2} \cap \theta$, with $\theta$ standing for the integrand polygonal path in the definition (1.13) of $T_{(\cdot)}^{[\beta]}[\cdot \leftrightarrow \cdot]$, it follows that

$$
T_{(\delta)}^{[\beta]}\left[0 \leftrightarrow\left(\lambda_{1}+\lambda_{2}+D\left[\lambda_{1}, \lambda_{2}\right]\right) \mathbf{e}_{x}\right] \geq
$$




$$
\mathrm{e}^{-O\left(D\left[\lambda_{1}, \lambda_{2}\right]\right)} T_{\left(\delta ; \Pi_{1}\right)}^{[\beta]}\left[0 \leftrightarrow \lambda_{1} \mathbf{e}_{x}\right] T_{\left(\delta ; \Pi_{2}\right)}^{[\beta]}\left[\left(\lambda_{1}+D\left[\lambda_{1}, \lambda_{2}\right]\right) \mathbf{e}_{x} \leftrightarrow\left(\lambda_{1}+\lambda_{2}+D\left[\lambda_{1}, \lambda_{2}\right]\right) \mathbf{e}_{x}\right],
$$

with the prefactor $\mathrm{e}^{-O\left(D\left[\lambda_{1}, \lambda_{2}\right]\right)}$ due to patching together pairs of paths $\theta_{1}$ in $\mathcal{C}^{0 \leftrightarrow \lambda_{1} \mathbf{e}_{x} ; \delta}$ and $\theta_{2}$ in $\mathcal{C}^{\left(\lambda_{1}+D\left[\lambda_{1}, \lambda_{2}\right]\right) \mathbf{e}_{x} \leftrightarrow\left(\lambda_{1}+\lambda_{2}+D\left[\lambda_{1}, \lambda_{2}\right]\right) \mathbf{e}_{x} ; \delta}$, both disjoint with $\mathcal{A}^{[\beta]}$, into paths $\theta$ falling into $\mathcal{C}^{0 \leftrightarrow\left(\lambda_{1}+\lambda_{2}+D\left[\lambda_{1}, \lambda_{2}\right]\right) \mathbf{e}_{x} ; \delta}$ disjoint with $\mathcal{A}^{[\beta]}$, by constructing a path connecting $\theta_{1}$ and $\theta_{2}$ across the moat of width $D\left[\lambda_{1}, \lambda_{2}\right]$ separating $\Pi_{1}$ and $\Pi_{2}$, according to a procedure completely analogous to that used in the argument leading to (5.50). Note that the fact that the patching procedure involves here conditioning on $\mathcal{A}^{[\beta]}$ being disjoint with $\theta_{1}$ and $\theta_{2}$ does not affect this argument because the conditional graphical construction of the process $\mathcal{A}_{\mathbb{R}^{2}: \theta_{1} \cup \theta_{2}}^{[[]}$guarantees that it is stochastically bounded by $\mathcal{P}_{\Theta[\beta]: \theta_{1} \cup \theta_{2}}$ and hence by $\mathcal{P}_{\Theta^{[\beta]}}$ as used in the proof of (5.50). To proceed, apply Lemma 5.2 to conclude that the quantities $T_{(\delta)}^{[\beta]}\left[0 \leftrightarrow \lambda_{1} \mathbf{e}_{x}\right]$ and $T_{(\delta)}^{[\beta]}\left[\left(\lambda_{1}+D\left[\lambda_{1}, \lambda_{2}\right]\right) \mathbf{e}_{x} \leftrightarrow\left(\lambda_{1}+\lambda_{2}+D\left[\lambda_{1}, \lambda_{2}\right]\right) \mathbf{e}_{x}\right]$ are bounded above by constant multiplicities of their respective finite volume counterparts $T_{\left(\delta ; \Pi_{1}\right)}^{[\beta]}\left[0 \leftrightarrow \lambda_{1} \mathbf{e}_{x}\right]$ and $T_{\left(\delta ; \Pi_{2}\right)}^{[\beta]}\left[\left(\lambda_{1}+D\left[\lambda_{1}, \lambda_{2}\right]\right) \mathbf{e}_{x} \leftrightarrow\left(\lambda_{1}+\lambda_{2}+D\left[\lambda_{1}, \lambda_{2}\right]\right) \mathbf{e}_{x}\right]$. Combining this conclusion with (5.52) shows that $T_{(\delta)}^{[\beta]}\left[0 \leftrightarrow\left(\lambda_{1}+\lambda_{2}+D\left[\lambda_{1}, \lambda_{2}\right]\right) \mathbf{e}_{x}\right] \geq \exp \left(-O\left(D\left[\lambda_{1}, \lambda_{2}\right]\right)\right) T_{(\delta)}^{[\beta]}\left[0 \leftrightarrow \lambda_{1} \mathbf{e}_{x}\right] T_{(\delta)}^{[\beta]}\left[\left(\lambda_{1}+\right.\right.$ $\left.\left.D\left[\lambda_{1}, \lambda_{2}\right]\right) \mathbf{e}_{x} \leftrightarrow\left(\lambda_{1}+\lambda_{2}+D\left[\lambda_{1}, \lambda_{2}\right]\right) \mathbf{e}_{x}\right]$ for some $C>0$, which completes the proof in view of the definition (1.14) of $\tau_{\lambda_{i}}^{[\beta]}, i=1,2$.

Completing the proof of Lemma 5.3 With the existence of the limit in (1.15) established we now easily conclude its strict positivity from the positivity of killing rate in the random walk representation (5.41) while the finiteness of $\tau^{[\beta]}$ follows by the probability lower bound (5.50).

\section{$6 \quad$ Skeleton estimates}

The purpose of this section is to provide coarse-graining estimates based on skeleton calculus. For $\alpha, \delta>0$, always assumed to satisfy $L \gg \alpha \gg \delta$ and to tend to $\infty$ as $L \rightarrow \infty$, by an $(\alpha, \delta)$-skeleton in $\mathbb{B}_{2}(L)$ we shall understand a collection $\left(I_{1}, E_{1}, I_{2}, E_{2}, \ldots, I_{m}, E_{m}\right)$ of pairwise different points (skeleton vertices) in $\mathbb{B}_{2}(L) \cap \mathbb{Z}^{2}$, with $I_{1}, I_{2}, \ldots$ referred to as the initial points, $E_{1}, E_{2}, \ldots$ as the corresponding endpoints and $\left[I_{1}, E_{1}\right],\left[I_{2}, E_{2}\right], \ldots$ as the skeleton segments, where the following is satisfied for all $i=1, \ldots, m$

(S1) $\alpha-\sqrt{2} \leq \operatorname{dist}\left(I_{i}, E_{i}\right) \leq \alpha+\sqrt{2}$. 
We say that a collection $\gamma$ of $\alpha$-large polygonal contours is compatible with an $(\alpha, \delta)$ skeleton $\Sigma=\left(I_{1}, E_{1}, \ldots, I_{m}, E_{m}\right)$, write $\gamma \sim \Sigma$, if the following holds for all $i=1, \ldots, m$

(S2) There exists a contour $\theta_{i} \in \gamma$ and points $I_{i}^{\gamma}, E_{i}^{\gamma} \in \theta_{i}$ such that $\operatorname{dist}\left(I_{i}, I_{i}^{\gamma}\right) \leq$ $\frac{1}{\sqrt{2}}, \operatorname{dist}\left(E_{i}, E_{i}^{\gamma}\right) \leq \frac{1}{\sqrt{2}}$ and $\operatorname{dist}\left(I_{i}, x\right) \leq \alpha+\frac{1}{\sqrt{2}}$ for all $x \in \theta_{i}\left[I_{i}^{\gamma}, E_{i}^{\gamma}\right]$ with $\theta_{i}\left[I_{i}^{\gamma}, E_{i}^{\gamma}\right]$ standing for the polygonal path from $I_{i}^{\gamma}$ to $E_{i}^{\gamma}$ along $\theta_{i}$ (note that we do not require that $\theta_{i} \neq \theta_{j}$ for $\left.i \neq j\right)$,

(S3) Either we have $\operatorname{dist}\left(I_{i},\left\{I_{1}, \ldots, I_{i-1}\right\}\right) \leq \alpha+\delta+\sqrt{2}$ or $i$ is the smallest index with $I_{i}^{\gamma} \in \theta_{i}$ for some $\theta_{i} \in \gamma$,

(S4) For each $x \in \gamma$ we have $\operatorname{dist}\left(x,\left\{I_{1}, \ldots, I_{m}\right\}\right) \leq 2 \alpha+\delta+\sqrt{2}$,

(S5) The polygonal paths $\theta_{i}\left[I_{i}^{\gamma}, E_{i}^{\gamma}\right]$ are in a distance at least $\delta$ away from each other.

Roughly speaking, the motivation underlying this definition is the following. For two distant points $x, y$ with dist $(x, y)=\Omega(\alpha)$ connected by a polygonal subpath of a contour we want to find a collection of approximately equal-sized segments $\left[I_{i}, E_{i}\right]$ of length $\alpha(1+o(1))$, lying on this path and such that their overall length is at least $\operatorname{dist}(x, y)(1+o(1))$. Being only concerned with this total length condition, as ensured by (S3) stating that the distance between initial points is close to the single segment length, we do not require that these segments form themselves a connected polygonal path or that their ordering agree with the orientation of the path. On the other hand, we do impose an explicit lower bound (S5) for distance between polygonal subpaths crossing different segments, thus ensuring the applicability of the decoupling Lemma 4.1 in our further argument. It should be emphasised that this approach, considerably simplifying our argument in the sequel, can only work in an isometry invariant setting, as ours, where it is justified to look only at the total length of phase interfaces while ignoring their local directions, ordering etc.

We say that a collection $\gamma$ of $\alpha$-large contours dominates an $(\alpha, \delta)$-skeleton $\Sigma$, write $\gamma \succeq \Sigma$, iff $\gamma$ contains a sub-family of contours $\gamma^{\prime}$ with $\gamma^{\prime} \sim \Sigma$. Further, by the length of a skeleton $\Sigma=\left(I_{1}, E_{1}, \ldots, I_{m}, E_{m}\right)$, denoted length $(\Sigma)$, we understand the total length of skeleton segments $\sum_{i=1}^{m} \operatorname{dist}\left(I_{i}, E_{i}\right)$. We write also $N(\Sigma)$ for the total number of initial and endpoints in $\Sigma$. We say that a collection $\gamma$ of $\alpha$-large polygonal contours is well covered by an $(\alpha, \delta)$-skeleton $\Sigma$, write $\gamma \propto \Sigma$, if the following holds

- $\gamma \sim \Sigma$, 
- $\Sigma$ maximises length $(\Sigma)$ among skeletons compatible with $\gamma$.

For an $(\alpha, \delta)$-skeleton $\Sigma$ we consider the corresponding black phase area, denoted in the sequel by $\operatorname{Area}(\Sigma)$, and given by

$$
\operatorname{Area}(\Sigma):=\sup _{\gamma \propto \Sigma} \operatorname{Area}\left(\operatorname{black}\left[\bigcup_{\theta \in \gamma} \theta\right]\right)
$$

In other words, Area $(\Sigma)$ is the supremum value of possible black phase area which can be enclosed by a collection $\gamma$ of $\alpha$-large contours well covered by $\Sigma$. We note that for some $\Sigma$ there may be no such $\gamma$ in which case we put by convention $\operatorname{Area}(\Sigma):=0$.

In the sequel when no ambiguity occurs we will often use the $\sim, \succeq$, $\propto$ notation for contour collection containing also $\alpha$-small contours, in which case we shall always mean that the approriate relation holds for the corresponding sub-ensemble of $\alpha$-large contours.

Lemma 6.1 For a collection $\gamma$ of $\alpha$-large contours there exists a compatible $(\alpha, \delta)$-skeleton $\Sigma$.

Proof Choose an initial point $I_{1} \in \mathbb{Z}^{2} \cap \mathbb{B}_{2}(L)$ at a distance less that $1 / \sqrt{2}$ from some $\theta_{1} \in \gamma$, set $I_{1}^{\gamma}$ to be the point of $\theta_{1}$ minimising the distance to $I_{1}$ and let $E_{1}^{\gamma}$ be the first point (say in clockwise order) on $\theta_{1}$ at the distance $\alpha$ from $I_{1}^{\gamma}$ (note that the distance considered here and below is the usual Euclidean distance and not the distance along the contour $\theta_{1}$ !). Set $E_{1}$ to be the point of $\mathbb{B}_{2}(L) \cap \mathbb{Z}^{d}$ which lies the closest to $E_{1}^{\gamma}$. The conditions $(\mathbf{S 1}),(\mathrm{S} 2)$ for $i=1$ is now easily verified. Further, if existing, choose $I_{2}^{\gamma}$ to be the point minimising the distance to $\theta_{1}\left[I_{1}^{\gamma}, E_{1}^{\gamma}\right]$ (with ties broken in an arbitrary way) among the points $I_{2}^{\gamma}$ in $\gamma$ with the property that there exists $E_{2}^{\gamma} \in \theta_{2}$ with $\operatorname{dist}\left(I_{2}^{\gamma}, E_{2}^{\gamma}\right)=\alpha$, $\operatorname{dist}\left(I_{2}^{\gamma}, x\right) \leq \alpha$ for all $x \in \theta_{2}\left[I_{2}^{\gamma}, E_{2}^{\gamma}\right]$ and $\operatorname{dist}\left(\theta_{1}\left[I_{1}^{\gamma}, E_{1}^{\gamma}\right], \theta_{2}\left[I_{2}^{\gamma}, E_{2}^{\gamma}\right]\right) \geq \delta$, where $\theta_{2}$ is the contour of $\gamma$ containing $I_{2}^{\gamma}$. Note that if $\theta_{1}=\theta_{2}$ then $\operatorname{dist}\left(I_{2}^{\gamma}, \theta_{1}\left[I_{1}^{\gamma}, E_{1}^{\gamma}\right]\right)=\delta$ and hence $\operatorname{dist}\left(I_{1}^{\gamma}, I_{2}^{\gamma}\right) \leq \alpha+\delta$. In case such $I_{2}^{\gamma}$ and $E_{2}^{\gamma}$ exist, we define $I_{2}$ and $E_{2}$ as the best approximations in $\mathbb{Z}^{2} \cap \mathbb{B}_{2}(L)$ of $I_{2}^{\gamma}$ and $E_{2}^{\gamma}$ respectively, getting the required relations $(\mathbf{S 1}),(\mathrm{S} 2),(\mathrm{S} 3),(\mathrm{S} 5)$ for $i=2$. On the other hand, if such a pair $\left(I_{2}^{\gamma}, E_{2}^{\gamma}\right)$ fails to exist, we conclude that no point of $\gamma$ lies further than $\alpha+\delta$ away from $\theta_{1}\left[I_{1}^{\gamma}, E_{1}^{\gamma}\right]$, for otherwise we could find $I_{2}^{\gamma}$ and $E_{2}^{\gamma}$ with desired properties. In this case by (S2) we have $\operatorname{dist}\left(x, I_{1}^{\gamma}\right) \leq 2 \alpha+\delta$ for all $x$ in $\gamma$ which yields (S4), and $(\mathbf{S 3}),(\mathbf{S 5})$ are obvious, whence $\left(I_{1}, E_{1}\right)$ is already an $(\alpha, \delta)$-skeleton compatible with $\gamma$. 
We proceed inductively with this construction, adding new pairs $\left(I_{i+1}, E_{i+1}\right)$ obtained as the best lattice approximations of $\left(I_{i+1}^{\gamma}, E_{i+1}^{\gamma}\right)$ with $I_{i+1}^{\gamma}$ arising as the point minimising the distance to $\bigcup_{j \leq i} \theta_{j}\left[I_{j}^{\gamma}, E_{j}^{\gamma}\right]$ among the points $I_{i+1}^{\gamma} \in \theta_{i+1} \in \gamma$ for which there exists $E_{i+1}^{\gamma} \in \theta_{i+1}$ with $\operatorname{dist}\left(I_{i+1}^{\gamma}, E_{i+1}^{\gamma}\right)=\alpha, \operatorname{dist}\left(I_{i+1}^{\gamma}, x\right) \leq \alpha$ for all $x \in \theta_{i+1}\left[I_{i+1}^{\gamma}, E_{i+1}^{\gamma}\right]$, and such that $\operatorname{dist}\left(\theta_{i+1}\left[I_{i+1}^{\gamma}, E_{i+1}^{\gamma}\right], \bigcup_{j \leq i} \theta_{j}\left[I_{j}^{\gamma}, E_{j}^{\gamma}\right]\right) \geq \delta$. We note that if $\theta_{i+1}=\theta_{j}$ for some $j \leq i$ then $\operatorname{dist}\left(I_{i+1}^{\gamma}, \bigcup_{j \leq i} \theta_{j}\left[I_{j}^{\gamma}, E_{j}^{\gamma}\right]\right)=\delta$ and hence $\operatorname{dist}\left(I_{i+1}^{\gamma},\left\{I_{1}^{\gamma}, \ldots, I_{i}^{\gamma}\right\}\right) \leq \alpha+\delta$. The construction terminates when no further pair can be found, and it is easily verified as in the argument above that the resulting collection $\left(I_{1}, E_{1}, I_{2}, E_{2}, \ldots\right)$ is an $(\alpha, \delta)$-skeleton compatible with $\gamma$. The proof is complete.

Recalling that, by the definition, skeletons have their vertices pairwise different and belonging to $\mathbb{B}_{2}(L) \cap \mathbb{Z}^{2}$ and hence their number is finite, we obtain the following corollary as an immediate conclusion of Lemma 6.1.

Corollary 6.2 Each finite collection $\gamma$ of $\alpha$-large contours can be well covered by some $(\alpha, \delta)$-skeleton $\Sigma$.

A particular feature of the notion of skeleton as introduced in this section is that if two polygonal subpaths of some contours go very close to each other, it may happen that only one of these subpaths will contribute to the total length of a well-covering skeleton because of the requirement that subpaths going along the segments of the skeleton keep distance at least $\delta$ from each other as imposed in (S5) above. However, this does not lead to problems in our further argument, since we are mainly concerned with minimising the skeleton length given the enclosed area, where collections consisting of multiple contours are outperformed by singleton ones. This is made formal in the isoperimetric lemma below.

Lemma 6.3 Assume that $A \ll \alpha^{6}$. Then for each $(\alpha, \delta)$-skeleton $\Sigma$ in $\mathbb{B}_{2}(L)$ with $\operatorname{Area}(\Sigma)=$ $A, A \in\left[0, \pi L^{2}\right]$, we have

$$
\text { length }(\Sigma) \geq 2 \sqrt{\pi A}[1-O(\delta / \alpha)]-O(\alpha)
$$

Proof Below, we restrict our attention to skeletons $\Sigma$ with length $(\Sigma) \leq 2 \pi L$, since otherwise our assertion is obvious.

Pick some collection of $\alpha$-large contours $\gamma^{*} \propto \Sigma$ with Area(black $\left.\left[\bigcup_{\theta^{*} \in \gamma^{*}} \theta^{*}\right]\right)=\operatorname{Area}(\Sigma)-$ $o(1)=A-o(1)$, and observe that, by the definition of the relation $\propto$, to prove the lemma 
it is enough to construct $\Sigma^{*}$ with $\gamma^{*} \sim \Sigma^{*}$ and such that, for $A \gg \alpha^{2}$,

$$
\operatorname{length}\left(\Sigma^{*}\right) \geq A \psi(A)
$$

for a non-increasing function $A \mapsto \psi(A)=\psi(A ; \alpha, \delta)$ with $A \mapsto A \psi(A)$ non-decreasing, satisfying

$$
\psi(A)=2 \sqrt{\pi / A}[1-O(\delta / \alpha)]-O(\alpha / A)
$$

(note that the statement of the lemma trivialises for $A=O\left(\alpha^{2}\right)$ ). Without loss of generality we can and do assume that $\gamma^{*}$ contains no nested contours, for otherwise we could simply remove the internal contours increasing the area enclosed by $\gamma^{*}$, proceed with the construction below for the so reduced $\gamma^{*}$ obtaining $\Sigma^{*}$ of required length, and then construct some additional skeleton segments for the internal contours and add them to $\Sigma^{*}$ thus increasing its length even further. We also assume that $\gamma^{*}$ contains only contours for which $\operatorname{dist}\left(\theta^{*}, \bigcup_{\delta^{*} \in \gamma^{*} \backslash\left\{\theta^{*}\right\}} \delta^{*}\right)>64 \alpha$. This does not result in loss of generality because finding a sub-collection $\hat{\gamma}^{*}$ of contours satisfying this condition and such that all other contours of $\gamma^{*}$ are contained in $64 \alpha$-neighbourhood of $\bigcup \hat{\gamma}^{*}$, and then constructing $\hat{\Sigma}^{*}$ for $\hat{\gamma}^{*}$, we see that the total area enclosed by the contours in $\gamma^{*} \backslash \hat{\gamma}^{*}$ is of order $O\left(\alpha \operatorname{length}\left(\hat{\Sigma}^{*}\right)\right)$, whence by (6.53) for $\hat{\Sigma}^{*}$ we get length $\left(\hat{\Sigma}^{*}\right) \geq\left[A-O\left(\alpha \operatorname{length}\left(\hat{\Sigma}^{*}\right)\right)\right] \psi\left(A-O\left(\alpha\right.\right.$ length $\left.\left(\hat{\Sigma}^{*}\right)\right)$ and consequently, by (6.54), length $\left(\Sigma^{*}\right) \geq \operatorname{length}\left(\hat{\Sigma}^{*}\right) \geq A \psi(A)-O(\alpha)$ provided $\alpha \operatorname{length}\left(\hat{\Sigma}^{*}\right)=$ $o(A)$. The remaining case length $\left(\hat{\Sigma}^{*}\right)=\Omega(A / \alpha)$ is easily handled directly, by considering subcases $A=O\left(\alpha^{2}\right)$ and $A \gg \alpha^{2}$.

The proof of existence of $\Sigma^{*}$ satisfying (6.53) goes by induction with respect to the number $n^{*}$ of contours in $\gamma^{*}=\left\{\theta_{1}^{*}, \ldots, \theta_{n^{*}}^{*}\right\}$, assumed to be ordered by decreasing enclosed area. For $n^{*}=1$ the assertion follows immediately by standard isoperimetric argument: note that the correcting term $A O(\alpha / A)=O(\alpha)$ coming to the RHS of (6.53) when substituting (6.54) is due to the admissible distance $\Theta(\alpha)$ between a skeleton and a compatible polygonal path [see (S4)], while the prefactor $1-O(\delta / \alpha)$ there comes from the fact that, in the single contour case, the distance between the initial point of a given skeleton segment and the set of preceding initial points may exceed the length of the segment by at most $\delta+2 \sqrt{2}$ [see (S1),(S3)], which is fraction $O(\delta / \alpha)$ of the segment length.

To proceed, take $n^{*}>1$. We split our argument into three possible cases.

Case 1: Say that a point $x \in \theta_{n^{*}}^{*}$ is $\alpha$-seen from a contour $\theta_{i}^{*} \in \gamma^{*}$ iff $\operatorname{dist}\left(x, \theta_{i}^{*}\right) \leq 4 \alpha$. Assume that the total length of the set $\operatorname{seen}\left(\theta_{n^{*}}^{*}, \theta_{i}^{*} ; \alpha\right)$ of all such points exceeds 
$16 \alpha$ for some $i<n^{*}$ and recall that, as assumed above, there exists $x \in \theta_{n^{*}}^{*}$ with $\operatorname{dist}\left(x, \bigcup_{j<n^{*}} \theta_{j}^{*}\right)>64 \alpha$. Patching $\theta_{n^{*}}^{*}$ and $\theta_{i}^{*}$ together with additional polygonal paths at two extreme points $x_{1}, x_{2}$ of $\operatorname{seen}\left(\theta_{n^{*}}^{*}, \theta_{i}^{*} ; \alpha\right)$ and removing the internal parts of both contours between $x_{1}$ and $x_{2}$, we replace $\theta_{n^{*}}^{*}$ and $\theta_{i}^{*}$ by a single contour $\theta_{+}^{*}$, which can be made disjoint with all remaining contours $\theta_{j}^{*}, j \neq i, j \neq n^{*}$. Denote by $\gamma_{+}^{*}$ the contour collection resulting from $\gamma^{*}$ by replacing $\theta_{n^{*}}^{*}$ and $\theta_{i}^{*}$ by $\theta_{+}^{*}$ and possibly removing some further contours which would become nested due to this replacement. It is easily seen that, by our assumptions above, any skeleton $\Sigma_{+}^{*} \sim \gamma_{+}^{*}$ can be modified into $\Sigma^{*} \sim \gamma^{*}$ with length $\left(\Sigma^{*}\right) \geq \operatorname{length}\left(\Sigma_{+}^{*}\right)$. Thus, the assertion (6.53) for $\gamma^{*}$ will follow if we are able to find such $\Sigma_{+}^{*}$ with length $\left(\Sigma_{+}^{*}\right) \geq A \psi(A)$. However, this is ensured by the inductive hypothesis in view of the relation Area $\left(\right.$ black $\left.\left[\bigcup_{\theta^{*} \in \gamma_{+}^{*}} \theta^{*}\right]\right) \geq$ Area $\left(\operatorname{black}\left[\bigcup_{\theta^{*} \in \gamma^{*}} \theta^{*}\right]\right)$.

Case 2: Next, suppose that length $\left(\operatorname{seen}\left(\theta_{n^{*}}^{*}, \theta_{i}^{*} ; \alpha\right)\right) \leq 16 \alpha$ for all $i<n^{*}$ and that $A_{n^{*}} \gg$ $\alpha^{2}$, where $A_{n^{*}}$ stands for the area enclosed by $\theta_{n^{*}}^{*}$. Recall in addition that there exists $x \in \theta_{n^{*}}^{*}$ with $\operatorname{dist}\left(x, \bigcup_{j<n^{*}} \theta_{j}^{*}\right)>64 \alpha$. We construct an $(\alpha, \delta)$-skeleton $\Sigma^{*}$ as follows. Put $\gamma_{-}^{*}:=\gamma^{*} \backslash\left\{\theta_{n^{*}}^{*}\right\}$ and observe that the area enclosed by $\gamma_{-}^{*}$ is $A-A_{n^{*}}-o(1)$, which is due to the fact that there is no contour nesting in $\gamma^{*}$ as assumed above. We let $\Sigma_{-}^{*}$ be an $(\alpha, \delta)$-skeleton such that

$$
\operatorname{length}\left(\Sigma_{-}^{*}\right) \geq\left[A-A_{n^{*}}\right] \psi\left(A-A_{n^{*}}\right) \geq\left[A-A_{n^{*}}\right] \psi(A)
$$

with its existence guaranteed by the inductive hypothesis [note that $A-A_{n^{*}} \gg \alpha^{2}$ since the contours are ordered by decreasing enclosed area]. The skeleton $\Sigma_{-}^{*}$ can be extended to a skeleton $\Sigma^{*}$ compatible with $\gamma^{*}$ by the procedure described in the proof of Lemma 6.1. Denoting by $\mathcal{S}^{*}:=\Sigma^{*} \backslash \Sigma_{-}^{*}$ the collection of newly added segments we see by our assumptions for Case 2 that $\mathcal{S}^{*}$ can be in its turn extended to an $(\alpha, \delta)$ skeleton $\hat{\mathcal{S}}^{*}$ compatible with $\left\{\theta_{n^{*}}^{*}\right\}$ by adding at most $O\left(n^{*}\right)$ new segments covering $\operatorname{seen}\left(\theta_{n^{*}}^{*}, \theta_{i}^{*} ; \alpha\right)$. Thus, using isoperimetric argument, as applied for the case $n^{*}=1$ above, we are led to

$$
\operatorname{length}\left(\mathcal{S}^{*}\right) \geq A_{n^{*}} \psi\left(A_{n^{*}}\right)-O\left(n^{*}\right)
$$

Recall now that the contours $\theta_{1}^{*}, \theta_{2}^{*}, \ldots$ are ordered by decreasing enclosed area, whence

$$
A_{n^{*}} \leq A / n^{*}
$$


Using (6.57) to rewrite (6.56) as length $\left(\mathcal{S}^{*}\right) \geq A_{n^{*}}\left[\psi\left(A_{n^{*}}\right)-O\left(n^{*} / A_{n^{*}}\right)\right] \geq A_{n^{*}}\left[\psi\left(A_{n^{*}}\right)\right.$ $\left.-O\left(A / A_{n^{*}}^{2}\right)\right]$ and then applying (6.54), noting that $A_{n^{*}} \leq A / 2$ by (6.57) and resorting to standard calculus in order to check that, for $\alpha$ large enough, we have $\psi\left(A_{n^{*}}\right)-O(A$ / $\left.A_{n^{*}}^{2}\right) \geq \psi(A)$ for $A_{n^{*}} \gg A^{2 / 3}$, we conclude from (6.56) that length $\left(\mathcal{S}^{*}\right) \geq A_{n^{*}} \psi(A)$ for $A_{n^{*}} \gg A^{2 / 3}$. On the other hand, the trivial bound length $\left(\mathcal{S}^{*}\right) \geq \alpha$ is easily seen to yield length $\left(\mathcal{S}^{*}\right) \geq A_{n^{*}} \psi(A)$ whenever $A_{n^{*}} \ll \alpha \sqrt{A}$. Since we assumed that $A \ll \alpha^{6}$ in the statement of the lemma, we get $\alpha \sqrt{A} \gg A^{2 / 3}$ which leads to

$$
\operatorname{length}\left(\mathcal{S}^{*}\right) \geq A_{n^{*}} \psi(A)
$$

for all $A_{n^{*}}$ within range of (6.57). Combining (6.58) with (6.55) and recalling that length $\left(\Sigma^{*}\right)=\operatorname{length}\left(\Sigma_{-}^{*}\right)+$ length $\left(\mathcal{S}^{*}\right)$ yields the required relation (6.53) for Case 2.

Case 3: Assume now that $A_{n^{*}}=O\left(\alpha^{2}\right)$. Then the required relation (6.53) can be obtained along the same lines as in Case 2 by recalling that $\operatorname{dist}\left(\theta_{n^{*}}^{*}, \bigcup_{j<n^{*}} \theta_{j}^{*}\right)>64 \alpha$ and noting that putting such $\theta_{n^{*}}^{*}$ into $\gamma^{*}$ results in large added length to added area ratio, exceeding $\psi(A)$. The only reason for discussing this case separately is the technical fact that $\psi\left(A_{n^{*}}\right)$ is formally not defined for $A_{n^{*}} \leq C \alpha^{2}$ unless $C$ is large enough.

The proof is now complete by induction.

With the concept of an $(\alpha, \delta)$-skeleton discussed above we are now in a position to proceed to the main result of this section.

Lemma 6.4 With $\alpha \rightarrow \infty, \delta \rightarrow \infty$ and $L \gg \alpha \gg \delta \gg \log L$, we eventually have for each $(\alpha, \delta)$-skeleton $\Sigma$ in $\mathbb{B}_{2}(L)$

$$
\mathbb{P}\left(\mathcal{A}^{[\beta]} \succeq \Sigma\right) \leq \exp \left(-\tau_{\alpha}^{[\beta]} \operatorname{length}(\Sigma)\right) .
$$

Proof For a contour collection $\gamma \succeq \Sigma, \gamma=\left\{\theta_{1}, \ldots, \theta_{k}\right\}$ we consider the partition $\Sigma[\gamma]=$ $\left\{\mathcal{S}\left[\theta_{1}\right], \ldots, \mathcal{S}\left[\theta_{k}\right]\right\}$ of $\Sigma$ into disjoint sub-skeletons $\mathcal{S}\left[\theta_{j}\right]$ composed of segments $[I, E]$ with the corresponding points $I^{\gamma}, E^{\gamma}$, as given by (S2), lying on $\theta_{j}$. Note that some $\mathcal{S}\left[\theta_{j}\right]$ may be empty. Moreover, for a non-empty sub-skeleton $\mathcal{S} \subseteq \Sigma$ we write $[\mathcal{S}]$ to denote the family of all contours $\theta$ such that $\operatorname{dist}(I, \theta) \leq 1 / \sqrt{2}$ and $\operatorname{dist}(E, \theta) \leq 1 / \sqrt{2}$ for all segments $[I, E] \in \mathcal{S}$. In particular, we always have $\theta_{j} \in\left[\mathcal{S}\left[\theta_{j}\right]\right]$ provided $\mathcal{S}\left[\theta_{j}\right] \neq \emptyset$. In view of $(1.6)$ or, equivalently, by the graphical construction of Section 1.2 , we see that

$$
\mathbb{P}\left(\mathcal{A}^{[\beta]} \succeq \Sigma\right) \leq \sum_{\left\{\mathcal{S}_{1}, \ldots, \mathcal{S}_{k}\right\}} \int_{\left[\mathcal{S}_{1}\right] \times \ldots \times\left[\mathcal{S}_{k}\right]} \mathbb{P}\left(\bigcup_{j=1}^{k} \theta_{j} \cap \mathcal{A}^{[\beta]}=\emptyset\right) 1_{\left\{\left\{\theta_{1}, \ldots, \theta_{k}\right\} \sim \Sigma\right\}} \prod_{j=1}^{k} d \Theta^{[\beta]}\left(\theta_{j}\right),
$$


where the sum ranges over all possible partitions $\left\{\mathcal{S}_{1}, \ldots, \mathcal{S}_{k}\right\}$ of $\Sigma$ and with the inequality rather than equality above due to the fact that we do not restrict the domain of integration to non-intersecting contours $\theta_{j}$ and that more than one contour of $\mathcal{A}^{[\beta]}$ might occur in $\left[\mathcal{S}_{j}\right]$, moreover it is not guaranteed that $\mathcal{S}_{j}=\mathcal{S}\left[\theta_{j}\right]$. We fix a partition $\Sigma=\mathcal{S}_{1} \cup \ldots \cup \mathcal{S}_{k}$ and, to distinguish between vertices coming from different sub-skeletons $\mathcal{S}_{j}, j=1, \ldots, k$, we subscript skeleton vertices with the corresponding sub-skeleton names, writing $I_{i ; \mathcal{S}_{j}}$ and $E_{i ; \mathcal{S}_{j}}$. We also put $\gamma:=\left\{\theta_{1}, \ldots, \theta_{k}\right\}$. Denote by $P_{i ; j}$ the polygonal subpath $\theta_{j}\left[\hat{I}_{i}^{\theta_{j}}, \hat{E}_{i}^{\theta_{j}}\right]$ of the contour $\theta_{j} \in\left[\mathcal{S}_{j}\right]$ in the above integral, with $\hat{I}_{i}^{\theta_{j}}$ and $\hat{E}_{i}^{\theta_{j}}$ standing for the points of $\theta_{j}$ closest to $I_{i ; \mathcal{S}_{j}}$ and $E_{i ; \mathcal{S}_{j}}$ respectively. Note that the points $\hat{I}_{i}^{\theta_{j}}$ and $\hat{E}_{i}^{\theta_{j}}$ do not have to coincide with $I_{i ; \mathcal{S}_{j}}^{\gamma}$ and $E_{i ; \mathcal{S}_{j}}^{\gamma}$ as specified by the correspondence (S2) implied by $\gamma \succeq \Sigma$; yet we clearly have $\operatorname{dist}\left(I_{i ; \mathcal{S}_{j}}^{\gamma}, \hat{I}_{i}^{\theta_{j}}\right) \leq \sqrt{2}$ and $\operatorname{dist}\left(E_{i ; \mathcal{S}_{j}}^{\gamma}, \hat{E}_{i}^{\theta_{j}}\right) \leq \sqrt{2}$. The reason for introducing $\hat{I}_{i}^{\theta_{j}}$ and $\hat{E}_{i}^{\theta_{j}}$ rather than simply using $I_{i ; \mathcal{S}_{j}}^{\gamma}$ and $E_{i ; \mathcal{S}_{j}}^{\gamma}$ in their stead is to ensure measurable dependence of $P_{i, j}$ on $\theta_{j}$.

Observe that by condition (S5) the distance between different $P_{i ; j}$ does not fall below $\delta$. Given the collections $(\theta):=\left(\theta_{j}\right)$ and $(P):=\left(P_{i ; j}\right)_{i, j}$ we consider the events

$$
\mathcal{I}_{i ; j}\left[\theta_{j}\right]:=\left\{\mathcal{A}^{[\beta]} \cap P_{i ; j}=\emptyset\right\}
$$

Taking into account that $\operatorname{Area}\left(P_{i, j} \oplus \mathbb{B}_{2}(1)\right)=O\left(\alpha^{2}\right)$ (see (S2)) and using the decoupling Lemma 4.1 yields uniformly in $(\theta)$

$$
\mathbb{P}\left(\bigcap_{i, j} \mathcal{I}_{i ; j}\left[\theta_{j}\right]\right)=\prod_{i, j}^{k} \mathbb{P}\left(\mathcal{I}_{i ; j}\left[\theta_{j}\right]\right)(1+O(\exp (-C \delta) \alpha \operatorname{length}(\Sigma) \log N(\Sigma))) .
$$

To proceed, note that, by (6.59),

$$
\mathbb{P}\left(\mathcal{A}^{[\beta]} \succeq \Sigma\right) \leq \sum_{\left\{\mathcal{S}_{1}, \ldots, \mathcal{S}_{k}\right\}} \int_{\left[\mathcal{S}_{1}\right] \times \ldots \times\left[\mathcal{S}_{k}\right]} \mathbb{P}\left(\bigcap_{i, j} \mathcal{I}_{i ; j}\left[\theta_{j}\right]\right) \mathbf{1}_{\left\{\left\{\theta_{1}, \ldots, \theta_{k}\right\} \sim \Sigma\right\}} \prod_{j=1}^{k} d \Theta^{[\beta]}\left(\theta_{j}\right)
$$

and hence, in view of (6.60), applying the rough bounds $N(\Sigma)=O\left(L^{2}\right), \alpha=O(L)$ and length $(\Sigma)=O\left(L^{3}\right)$ we obtain

$$
\begin{aligned}
\mathbb{P}\left(\mathcal{A}^{[\beta]} \succeq \Sigma\right) \leq & \sum_{\left\{\mathcal{S}_{1}, \ldots, \mathcal{S}_{k}\right\}} \int_{\left[\mathcal{S}_{1}\right] \times \ldots \times\left[\mathcal{S}_{k}\right]} \prod_{i, j} \mathbb{P}\left(\mathcal{I}_{i ; j}\left[\theta_{j}\right]\right) \prod_{j=1}^{k} d \Theta^{[\beta]}\left(\theta_{j}\right) \\
& \left(1+O\left(\exp (-C \delta) L^{4} \log L\right)\right) .
\end{aligned}
$$


For an endpoint $E_{i ; \mathcal{S}_{j}}$ we write $\varsigma\left(E_{i ; \mathcal{S}_{j}}\right)$ to denote the skeleton vertex $I_{i^{\prime} ; \mathcal{S}_{j}}$ or $I_{i^{\prime} ; \mathcal{S}_{j}}$ directly succeeding $E_{i ; \mathcal{S}_{j}}$ in clockwise order on $\theta_{j}$. Then, by the formulae (1.13) and (5.37) for $T_{(\cdot)}^{[\beta]}[\cdot \leftrightarrow \cdot]$ and $\vartheta_{(\cdot)}^{[\beta]}[\cdot \leftrightarrow \cdot]$ respectively, in view of the definitions of $\hat{T}_{(\cdot)}^{[\beta]}[\cdot \leftrightarrow \cdot]$ and $\hat{\vartheta}_{(\cdot)}^{[\beta]}[\cdot \leftrightarrow \cdot]$ as provided in Subsection 5.1, and by the definition (1.4) of the free contour measure and (1.11) of the free path measure we are led to

$$
\begin{gathered}
\mathbb{P}\left(\mathcal{A}^{[\beta]} \succeq \Sigma\right) \leq \sum_{\left\{\mathcal{S}_{1}, \ldots, \mathcal{S}_{k}\right\}} \sum_{\varsigma} \prod_{i, j}\left(\hat{T}_{\left(\frac{1}{\sqrt{2}}\right)}^{[\beta]}\left[I_{i ; \mathcal{S}_{j}} \leftrightarrow E_{i ; \mathcal{S}_{j}}\right] \hat{\vartheta}_{\left(\frac{1}{\sqrt{2}}\right)}^{[\beta]}\left[E_{i ; \mathcal{S}_{j}} \leftrightarrow \varsigma\left(E_{i ; \mathcal{S}_{j}}\right)\right]\right) \\
\left(1+O\left(\exp (-C \delta) L^{4} \log L\right)\right) C_{1}^{N(\Sigma)}, C_{1}>0,
\end{gathered}
$$

where the inner sum ranges over all possible successor assignments $\varsigma$ and where the extra factor $C_{1}^{N(\Sigma)}$ comes from integrating out the configuration of contours $\theta_{j}$ within $1 / \sqrt{2}$ neighbourhoods of $I_{i ; \mathcal{S}_{j}}$ and $E_{i ; \mathcal{S}_{j}}, i=1, \ldots$, which are subject to optimisation rather than integration in definitions of $\hat{T}_{(\cdot)}^{[\beta]}[\cdot \leftrightarrow \cdot]$ and $\hat{\vartheta}_{(\cdot)}^{[\beta]}[\cdot \leftrightarrow \cdot]$. Recall that $\operatorname{dist}\left(E_{i ; \mathcal{S}_{j}}, \varsigma\left(E_{i ; \mathcal{S}_{j}}\right)\right)>$ $\delta-\sqrt{2}$ in view of (S5) and then use the random walk representation of Lemma 5.1 and (5.40) combined with (5.38) to conclude that $\hat{\vartheta}_{\left(\frac{1}{\sqrt{2}}\right)}^{[\beta]}[x \leftrightarrow y]=\exp (-\Omega(\delta))$. Thus, taking into account that both the total number of possible partitions $\left\{\mathcal{S}_{1}, \ldots, \mathcal{S}_{k}\right\}$ and the total number of possible successor assignments $\varsigma$ are of order $\exp (O(N(\Sigma) \log N(\Sigma)))$, in view of (1.14) the relation (6.61) combined with (5.35) gives us

$$
\begin{gathered}
\mathbb{P}\left(\mathcal{A}^{[\beta]} \succeq \Sigma\right) \leq \prod_{i, j} \exp \left(-\tau_{\alpha}^{[\beta]} \operatorname{dist}\left(I_{i ; \mathcal{S}_{j}}, E_{i ; \mathcal{S}_{j}}\right)+O(N(\Sigma) \log N(\Sigma)-\Omega(\delta N(\Sigma)))\right) \\
\left(1+O\left(\exp (-C \delta) L^{4} \log L\right)\right) .
\end{gathered}
$$

Since, by definition, length $(\Sigma)=\sum_{i, j} \operatorname{dist}\left(I_{i ; \mathcal{S}_{j}}, E_{i ; \mathcal{S}_{j}}\right)$ and, moreover, $\exp (-C \delta) L^{4} \log L=$ $o(1)$ and $\delta N(\Sigma) \gg N(\Sigma) \log N(\Sigma)$ by the assumptions of the lemma, we conclude that

$$
\mathbb{P}\left(\mathcal{A}^{[\beta]} \succeq \Sigma\right) \leq \exp \left(-\tau_{\alpha}^{[\beta]} \operatorname{length}(\Sigma)\right)
$$

for $\alpha, \delta, L$ large enough, as required.

\section{Lower bound}

Below, we provide a lower bound for the occurrence probabilities of large contours in $\mathcal{A}^{[\beta]}$. This is complementary to the upper bounds obtained in the preceding Section 6. For $\alpha, \delta>0$ and for a piecewise smooth closed curve $\sigma$ in $\mathbb{R}^{2}$ we consider the event $\mathcal{U}[\sigma ; \alpha]$ 
that there exists a contour $\theta \in \mathcal{A}^{[\beta]}$ such that $\rho_{H}(\sigma, \theta) \leq 2 \alpha$ with $\rho_{H}(\cdot, \cdot)$ standing for the usual Hausdorff distance. The following lemma gives a lower bound for the probability of such event for $\sigma:=\mathbb{S}_{1}(R)=\partial \mathbb{B}_{2}(R)$.

Lemma 7.1 With $\alpha \rightarrow \infty, \delta \rightarrow \infty, R \rightarrow \infty$ such that $\log R \ll \delta \ll \alpha \ll R$ we have

$$
\mathbb{P}\left(\mathcal{U}\left[\mathbb{S}_{1}(R) ; \alpha\right]\right) \geq \exp \left(-2 \pi R \tau_{\alpha}^{[\beta]}-O(\delta R / \alpha)\right)
$$

Proof Note that

$$
\mathbb{P}\left(\mathcal{U}\left[\mathbb{S}_{1}(R) ; \alpha\right]\right) \geq \int_{\left\{\theta \in \mathcal{C} \mid \rho_{H}\left(\theta, \mathbb{S}_{1}(R)\right) \leq 2 \alpha\right\}} \mathbb{P}\left(\theta \cap \mathcal{A}^{[\beta]}=\emptyset\right) \Theta^{[\beta]}(d \theta)-\mathbb{P}\left(\mathcal{U}^{(>1)}\left[\mathbb{S}_{1}(R) ; \alpha\right]\right)
$$

where $\mathcal{U}^{(>1)}\left[\mathbb{S}_{1}(R) ; \alpha\right]$ is the event that there exist at least two contours $\theta_{1}, \theta_{2}, \ldots$ in $\mathcal{A}^{[\beta]}$ such that $\rho_{H}\left(\mathbb{S}_{1}(R), \theta_{i}\right) \leq 2 \alpha, i=1,2, \ldots$ Using the conditional graphical construction with forbidden regions we easily see that $\mathbb{P}\left(\mathcal{U}^{(>1)}\left[\mathbb{S}_{1}(R) ; \alpha\right] \mid \mathcal{U}\left[\mathbb{S}_{1}(R) ; \alpha\right]\right)=o(1)$, whence (7.62) becomes

$$
\mathbb{P}\left(\mathcal{U}\left[\mathbb{S}_{1}(R) ; \alpha\right]\right) \geq \int_{\left\{\theta \in \mathcal{C} \mid \rho_{H}\left(\theta, \mathbb{S}_{1}(R)\right) \leq 2 \alpha\right\}} \mathbb{P}\left(\theta \cap \mathcal{A}^{[\beta]}=\emptyset\right) \Theta^{[\beta]}(d \theta)(1-o(1)) .
$$

To proceed, we partition the circle $\mathbb{S}_{1}(R)$ into disjoint segments $\left[I_{i}, E_{i}\right], i=1, \ldots, N(R ; \alpha, \delta)$ $=\Theta(R / \alpha)$ separated by spacings of length $\delta$ and such that $\operatorname{dist}\left(I_{i}, E_{i}\right)=\alpha, i=1, \ldots$, $N(R ; \alpha, \delta)$. Denote by $\Pi_{i}$ the square $\Pi\left(I_{i} \leftrightarrow E_{i} ; 1 / \sqrt{2}\right)$ as defined in the lines preceding Lemma 5.2. Clearly, $\Pi_{i}$ are disjoint and $\operatorname{dist}\left(\Pi_{i}, \Pi_{j}\right)=\Theta(\delta)$ for $i \neq j$. The integral in (7.63) can be bounded below by restricting the domain of integration to the family $\mathcal{C}\left[I_{1}, E_{1}, \ldots\right]$ of paths $\theta$ such that, for all $i=1, \ldots, N(R ; \alpha, \delta), \theta$ contains a subpath $\theta_{i}$ connecting $\partial \mathbb{B}_{2}\left(I_{i}, 1 / \sqrt{2}\right)$ to $\partial \mathbb{B}_{2}\left(E_{i}, 1 / \sqrt{2}\right)$ within $\Pi_{i}$. Using the decoupling Lemma 4.1, with $U_{i}:=\theta \cap \Pi_{i}$ there, we can factorize the integral

$$
\int_{\left\{\theta \in \mathcal{C}\left[I_{1}, E_{1}, \ldots\right] \mid \rho_{H}\left(\theta, \mathbb{S}_{1}(0, R)\right) \leq 2 \alpha\right\}} \mathbb{P}\left(\theta \cap \mathcal{A}^{[\beta]}=\emptyset\right) \Theta^{[\beta]}(d \theta)
$$

into the product of $T_{\left(1 / \sqrt{2} ; \Pi_{i}\right)}^{[\beta]}\left[I_{i} \leftrightarrow E_{i}\right], i=1, \ldots, N(R ; \alpha, \delta)$ with a prefactor

$$
\left(1+O\left(R \exp \left(-C_{1} \delta\right) \log N(R ; \alpha, \delta)\right)\right) \exp (O(\delta N(R ; \alpha, \delta))), C_{1}>0
$$

where $\left(1+O\left(R \exp \left(-C_{1} \delta\right) \log N(R ; \alpha, \delta)\right)\right)$ is the factorization correction from Lemma 4.1 while $\exp (O(\delta N(R ; \alpha, \delta)))$ comes from patching the contour $\theta$ by joining together the 
subpaths $\theta_{i}$ passing through adjacent $\delta$-distant squares $\Pi_{i}$ so as to keep the resulting path within distance $2 \alpha$ from $\mathbb{S}_{1}(R)$, see the discussion of (5.52) and (5.50) above. Since $R \exp \left(-C_{1} \delta\right) \log N(R ; \alpha, \delta)=o(1)$, we obtain

$$
\mathbb{P}\left(\mathcal{U}\left[\mathbb{S}_{1}(R) ; \alpha\right]\right) \geq \exp (O(\delta N(R ; \alpha, \delta))) \prod_{i=1}^{N(R ; \alpha, \delta)} T_{\left(1 / \sqrt{2} ; \Pi_{i}\right)}^{[\beta]}\left[I_{i} \leftrightarrow E_{i}\right] .
$$

Applying Lemma 5.2 we conclude that

$$
\mathbb{P}\left(\mathcal{U}\left[\mathbb{S}_{1}(R) ; \alpha\right]\right) \geq O\left(C_{2}^{N(R ; \alpha, \delta)}\right) \exp (O(\delta N(R ; \alpha, \delta))) \prod_{i=1}^{N(R ; \alpha, \delta)} T_{(1 / \sqrt{2})}^{[\beta]}\left[I_{i} \leftrightarrow E_{i}\right], C_{2}>0 .
$$

Observing that $N(R ; \alpha, \delta)=\Theta(R / \alpha)$ completes the proof in view of the definition (1.14) of $\tau_{\alpha}^{[\beta]}$.

\section{Proof of the main theorem}

Throughout this proof we shall put

$$
\alpha=\alpha[L]:=\sqrt{L} \log L \text { and } \delta=\delta[L]:=(\log L)^{2} .
$$

As in the classical DKS theory, our argument below uses the decomposition of the contour ensemble $\mathcal{A}^{[\beta]} \cap \mathbb{B}_{2}(L)$ into the collection $\mathbb{L}_{\alpha ; L}:=\mathbb{L}_{\alpha ; L}\left[\mathcal{A}^{[\beta]}\right]$ of $\alpha$-large contours and the remaining family of $\alpha$-small contours, and it relies on an application of the skeleton bounds of Section 6 and complementary estimates of Section 7, combined with the use of moderate deviation results of Section 3.

\subsection{Lower bound for (1.17)}

In order to prove (1.17) we establish first the lower bound

$$
\mathbb{P}\left(\mathbb{M}_{L}\left(\mathcal{A}^{[\beta]}\right) \geq \mathbb{M}[\beta] \pi L^{2}+a L^{2}, \mathcal{N}[\alpha ; L] \text { holds }\right) \geq \exp \left(-\sqrt{\frac{2 \pi a}{|\mathbb{M}[\beta]|}} L \tau_{\alpha}^{[\beta]}+O(\alpha)\right)_{(8.65)}
$$

To show it, put

$$
R:=L \sqrt{\frac{a}{2 \pi|\mathbb{M}[\beta]|}}+C \alpha
$$


for some constant $C$ large enough so that

$$
\mathbb{P}\left(\mathbb{M}_{L}\left(\mathcal{A}^{[\beta]}\right) \geq \mathbb{M}[\beta] \pi L^{2}+a L^{2} \mid \mathcal{U}\left[\mathbb{S}_{1}(R) ; \alpha\right]\right)>1 / 2,
$$

with the event $\mathcal{U}\left[\mathbb{S}_{1}(R) ; \alpha\right]$, indicating the existence of a contour $\theta$ of $\mathcal{A}^{[\beta]}$ with $\rho_{H}\left(\theta, \mathbb{S}_{1}(R)\right)$ $\leq 2 \alpha$, defined as in Section 7. Clearly, $R<L$ for $L$ large enough because $a<2 \pi|\mathbb{M}[\beta]|$. To see that the required choice of $C$ in (8.66) is indeed possible note first that

$$
\begin{gathered}
\mathbb{P}\left(\mathbb{M}_{L}\left(\mathcal{A}^{[\beta]}\right) \leq \mathbb{M}[\beta] \pi L^{2}+a L^{2} \mid \mathcal{U}\left[\mathbb{S}_{1}(R) ; \alpha\right]\right) \leq \\
\frac{1}{\mathbb{P}\left(\mathcal{U}\left[\mathbb{S}_{1}(R) ; \alpha\right]\right)} \int_{\left\{\theta \in \mathcal{C}, \rho_{H}\left(\theta, \mathbb{S}_{1}(R)\right) \leq 2 \alpha\right\}} \mathbb{P}\left(\mathbb{M}_{L}\left(\mathcal{A}_{\mathbb{R}^{2} ; \theta}^{[\beta]} \cup \theta\right) \leq \mathbb{M}[\beta] \pi L^{2}+a L^{2}\right) \Theta^{[\beta]}(d \theta) .
\end{gathered}
$$

Then use (3.20) to conclude that, for $\rho_{H}\left(\theta, \mathbb{S}_{1}(R)\right) \leq 2 \alpha$,

$$
\mathbb{E} \mathbb{M}_{L}\left(\mathcal{A}_{\mathbb{R}^{2} ; \theta}^{[\beta]} \cup \theta\right)=\mathbb{M}[\beta] \pi L^{2}+a L^{2}+4 \pi|\mathbb{M}[\beta]| C R \alpha+O(L \alpha),
$$

which can be made larger than $\mathbb{M}[\beta] \pi L^{2}+a L^{2}$ by a term of order $\Theta(L \alpha)$ under appropriate choice of $C$. In view of Corollary 3.3 this makes the integrand probability $\mathbb{P}\left(\mathbb{M}_{L}\left(\mathcal{A}_{\mathbb{R}^{2} ; \theta}^{[]]} \cup\right.\right.$ $\left.\theta) \leq \mathbb{M}[\beta] \pi L^{2}+a L^{2}\right)$ arbitrarily close to 0 , uniformly in $\theta$ with $\rho_{H}\left(\mathbb{S}_{1}(R), \theta\right) \leq 2 \alpha$. In particular, (8.67) is seen to hold under such choice of $C$, as required. To proceed, observe that the conditional probability $\mathbb{P}\left(\mathcal{N}^{c}[\alpha ; L] \mid \mathcal{U}\left[\mathbb{S}_{1}(R) ; \alpha\right]\right)$, being bounded above by $\left[\mathbb{P}\left(\mathcal{U}\left[\mathbb{S}_{1}(R) ; \alpha\right]\right)\right]^{-1} \int_{\left\{\theta \in \mathcal{C}, \rho_{H}\left(\theta, \mathbb{S}_{1}(R)\right) \leq 2 \alpha\right\}} \mathbb{P}\left(\mathcal{N}^{c}[\alpha ; L]\right.$ holds for $\left.\mathcal{A}_{\mathbb{R}^{2} ; \theta}^{[\beta]}\right) \Theta^{[\beta]}(d \theta)$, tends to 0 as $L \rightarrow \infty$ by the results of Lemma 2.1 in Section 2 specialised for $\mathcal{A}_{\mathbb{R}^{2} ; \theta}^{[\beta]}$. Thus, we conclude from (8.67) that for sufficiently large $L$

$$
\mathbb{P}\left(\mathbb{M}_{L}\left(\mathcal{A}^{[\beta]}\right) \geq \mathbb{M}[\beta] \pi L^{2}+a L^{2}, \mathcal{N}[\alpha ; L] \text { holds } \mid \mathcal{U}\left[\mathcal{S}_{1}(R) ; \alpha\right]\right)>1 / 4
$$

The required relation (8.65) follows now by Lemma 7.1 in view of (8.66).

\subsection{Upper bound for (1.17)}

To complete the proof of (1.17) we shall establish the following upper bound, complementary to $(8.65)$,

$$
\mathbb{P}\left(\mathbb{M}_{L}\left(\mathcal{A}^{[\beta]}\right) \geq \mathbb{M}[\beta] \pi L^{2}+a L^{2}, \mathcal{N}[\alpha ; L] \text { holds }\right) \leq \exp \left(-\sqrt{\frac{2 \pi a}{|\mathbb{M}[\beta]|}} L \tau_{\alpha}^{[\beta]}+O(\alpha)\right)
$$


To this end use the exponential tightness bound in Lemma 2.1 to get for some $C_{1}=C_{1}(a)$ and $C_{2}>\sqrt{\frac{2 \pi a}{|\mathbb{M}[\beta]|}} \tau^{[\beta]}$

$$
\begin{gathered}
\mathbb{P}\left(\mathbb{M}_{L}\left(\mathcal{A}^{[\beta]}\right) \geq \mathbb{M}[\beta] \pi L^{2}+a L^{2}, \mathcal{N}[\alpha ; L] \text { holds }\right) \leq \\
\mathbb{P}\left(\mathbb{M}_{L}\left(\mathcal{A}^{[\beta]}\right) \geq \mathbb{M}[\beta] \pi L^{2}+a L^{2}, \text { length }\left(\mathbb{L}_{\alpha ; L}\right) \leq C_{1} L, \mathcal{N}[\alpha ; L] \text { holds }\right)+O\left(\exp \left(-C_{2} L\right)\right)
\end{gathered}
$$

Applying Lemma 6.1 together with Corollary 6.2 we see that this probability is bounded above by

$$
\sum_{\Sigma} \mathbb{P}\left(\mathbb{M}_{L}\left(\mathcal{A}^{[\beta]}\right) \geq \mathbb{M}[\beta] \pi L^{2}+a L^{2}, \operatorname{length}\left(\mathbb{L}_{\alpha ; L}\right) \leq C_{1} L, \mathbb{L}_{\alpha ; L} \propto \Sigma\right)+O\left(\exp \left(-C_{2} L\right)\right)
$$

where the sum above is taken over all $(\alpha, \delta)$-skeletons $\Sigma$ contained in $\mathbb{B}_{2}(L-[4 \alpha-\delta-\sqrt{2}])$. Note that we could restrict our attention to $\Sigma \subseteq \mathbb{B}_{2}(L-[4 \alpha-\delta-\sqrt{2}])$ because of working on the event $\mathcal{N}[\alpha, L]$, see (S4). It should also be noted that any contour collection well covered by such $\Sigma$ is completely contained in $\mathbb{B}_{2}(L)$ for $L$ and $\alpha[L]$ large enough. Under the imposed requirement that length $\left(\mathbb{L}_{\alpha ; L}\right) \leq C_{1} L$, the total length of skeleton segments in any $\Sigma$ with $\mathbb{P}\left(\mathbb{L}_{\alpha ; L} \propto \Sigma\right)>0$ is also of order $O(L)$, whence the sum in (8.69) can be restricted only to skeletons of such length order. Observe now that the number of such skeletons is of order $\exp \left(O\left(\alpha^{-1} L \log L\right)\right)$ which, by $(8.64)$, is $\exp (O(\sqrt{L}))$. Indeed, constructing the skeleton segment after segment at each step we have at most $O\left(L^{2}\right)$ possibilities of choosing new initial/end point. However, the total number of such steps, coinciding with twice the number of segments, is at most of order $O(L / \alpha)$, because, as stated above, we only consider skeletons $\Sigma$ with length $(\Sigma)=O(L)$ and the length of a single segment is close to $\alpha$, see (S1). We put this statement as a remark for further reference

Remark 8.1 The number of $(\alpha, \delta)$-skeletons $\Sigma$ in $\mathbb{B}_{2}(L)$ with length $(\Sigma)=O(L)$ is of order $\exp \left(O\left(\alpha^{-1} L \log L\right)\right)$.

Consequently, by (8.69), in order to establish (8.68) it is enough to show that

$$
\begin{aligned}
\max _{\Sigma} \mathbb{P}\left(\mathbb{M}_{L}\left(\mathcal{A}^{[\beta]}\right)\right. & \left.\geq \mathbb{M}[\beta] \pi L^{2}+a L^{2}, \operatorname{length}\left(\mathbb{L}_{\alpha ; L}\right) \leq C_{1} L, \mathbb{L}_{\alpha ; L} \propto \Sigma\right) \\
& \leq \exp \left(-\sqrt{\frac{2 \pi a}{|\mathbb{M}[\beta]|}} L \tau_{\alpha}^{[\beta]}+O(\alpha)\right),
\end{aligned}
$$


with the maximum taken over all $(\alpha, \delta)$-skeletons $\Sigma$ satisfying the conditions specified above (i.e. contained in $\mathbb{B}_{2}(L-[4 \alpha-\delta-\sqrt{2}])$ and with total length of order $\left.O(L)\right)$. To proceed with the verification of $(8.70)$ choose a skeleton $\Sigma_{0}$ which achieves the above maximum. Putting $\nu:=\operatorname{Area}\left(\Sigma_{0}\right)$ and $\lambda:=\operatorname{length}\left(\Sigma_{0}\right)$ we conclude by the isoperimetric Lemma 6.3 that

$$
\lambda \geq 2 \sqrt{\pi \nu}[1-O(\delta / \alpha)]-O(\alpha) .
$$

Using that $\nu=O\left(L^{2}\right)$ and that $\delta L / \alpha=\alpha$ we obtain

$$
\lambda \geq 2 \sqrt{\pi \nu}-O(\alpha)
$$

To proceed, recall that $\mathbb{M}[\beta] \in(-1,0)$ and observe that on the event $\mathbb{L}_{\alpha ; L} \propto \Sigma_{0}$ we get by (3.20) and (S4)

$$
\mathbb{E} \mathbb{M}_{L}\left(\mathcal{A}_{\mathbb{R}^{2}: \gamma}^{[\beta] \alpha, \mathbb{B}_{2}(L)} \cup \gamma\right) \leq \mathbb{M}[\beta]\left(\pi L^{2}-\nu\right)-\nu \mathbb{M}[\beta]+O(L \alpha) .
$$

Thus, noting that the field $\mathcal{A}^{[\beta]}$ conditioned on $\mathbb{L}_{\alpha ; L}=\gamma$ coincides in law with $\mathcal{A}_{\mathbb{R}^{2}: \gamma}^{[\beta] \alpha, \mathbb{B}_{2}(L)} \cup \gamma$ and recalling that $N(\Sigma)=O(\lambda / \alpha)$, we conclude from Lemma 6.4 and Theorem 3.1 applied conditionally on $\mathbb{L}_{\alpha ; L}$ that the probability maximised in (8.70) is bounded above by

$$
\exp \left(-\tau_{\alpha}^{[\beta]} \lambda\right) \exp \left(-c\left[\frac{\Delta^{2}}{L^{2}} \wedge \frac{\Delta}{\alpha}\right]\right)
$$

where $\Delta:=\left[\mathbb{M}[\beta]\left(\pi L^{2}-\nu\right)-\nu \mathbb{M}[\beta]+O(L \alpha)\right]-\left[\mathbb{M}[\beta] \pi L^{2}+a L^{2}\right]=2 \nu|\mathbb{M}[\beta]|-a L^{2}+O(L \alpha)$ is the difference between the expected and actual (required) magnetisation on the event $\mathbb{L}_{\alpha ; L} \propto \Sigma_{0}$, and with $\nu$ and $\lambda$ related by (8.71). Recalling that $\lambda=O(L), \alpha=\sqrt{L} \log L$, $\delta=(\log L)^{2}$ and applying the lower bound (8.65) we see that the maximum in (8.72) has to be reached with $\Delta=O\left(L^{3 / 2} \log L\right)=O(L \alpha)$ and, consequently,

$$
\nu=\frac{a L^{2}}{2|\mathbb{M}[\beta]|}+O(L \alpha)
$$

whence, by (8.71),

$$
\lambda=L \sqrt{\frac{2 \pi a}{|\mathbb{M}[\beta]|}}+O(\alpha),
$$

with the equality rather than inequality in the last formula due to (8.65). By Lemma 6.4 this yields the required relation (8.70) and hence completes the proof of (8.68). 


\subsection{Existence of a large contour}

In view of the lower bound (8.65), the argument leading to (8.72) with the optimal skeleton $\Sigma_{0}$ replaced by a generic skeleton $\Sigma$ shows that, conditionally on the event $\left\{\mathbb{M}_{L}\left(\mathcal{A}^{[\beta]}\right)>\mathbb{M}[\beta] \pi L^{2}+a L^{2}, \mathcal{N}[\alpha, L]\right.$ holds $\}$, with probability tending to 1 we can have $\mathbb{L}_{\alpha, L} \propto \Sigma$ only for those $(\alpha, \delta)$-skeletons $\Sigma$ which satisfy (8.73) and (8.74) with $\nu=\operatorname{Area}(\Sigma)$ and $\lambda=$ length $(\Sigma)$. By the definition of the relation $\propto$ and by the proof of the isoperimetric Lemma 6.3 this means that with conditional probability tending to 1 on the event $\left\{\mathbb{M}_{L}\left(\mathcal{A}^{[\beta]}\right)>\mathbb{M}[\beta] \pi L^{2}+a L^{2}, \mathcal{N}[\alpha, L]\right.$ holds $\}$ there exists at least one contour $\theta_{\text {large }}$ of length $L \sqrt{\frac{2 \pi a}{|\mathbb{M}[\beta]|}}+O(\alpha)$ and enclosing area $\frac{a L^{2}}{2|\mathbb{M}[\beta]|}+O(L \alpha)$. In fact, we claim that for $K$ large enough, conditionally on $\left\{\mathbb{M}_{L}\left(\mathcal{A}^{[\beta]}\right)>\mathbb{M}[\beta] \pi L^{2}+a L^{2}, \mathcal{N}[\alpha, L]\right.$ holds $\}$, with probability arbitrarily close to 1 the contour $\theta_{\text {large }}$ is the only $K \alpha$-large contour of $\mathcal{A}^{[\beta]}$ in $\mathbb{B}_{2}(L)$. Indeed, for each $\Sigma$ as above, i.e. satisfying (8.73) and (8.74), we have

$\mathbb{P}\left(\mathbb{L}_{\alpha, L} \propto \Sigma, \mathcal{A}^{[\beta]}\right.$ contains more than one $K \alpha$-large contour in $\mathbb{B}_{2}(L), \mathcal{N}[\alpha, L]$ holds $)$

$$
\leq \int_{\theta_{\text {large }}} \mathbb{P}\left(\text { length } \mathbb{L}_{\alpha, L}\left(\mathcal{A}_{\mathbb{R}^{2}: \theta_{\text {large }}}^{[\beta]}\right) \geq K \alpha\right) \Theta^{[\beta]}\left(d \theta_{\text {large }}\right),
$$

where the integral ranges over $\theta_{\text {large }}$ in $\mathbb{B}_{2}(L)$ of length $L \sqrt{\frac{2 \pi a}{|\mathbb{M}[\beta]|}}+O(\alpha)$ and enclosing area $\frac{a L^{2}}{2|\mathbb{M}[\beta]|}+O(L \alpha)$. Now, Remark 8.1 and Lemma 6.4 imply that the total mass $\Theta^{[\beta]}(\cdot)$ of such $\theta_{\text {large }}$ 's is of order $\exp \left(-\sqrt{\frac{2 \pi a}{\mid \mathbb{M}[\beta]}} L \tau_{\alpha}^{[\beta]}+O(\alpha)\right)$. Moreover, by Lemma 2.1 applied to $\mathcal{A}_{\mathbb{R}^{2}: \theta_{\text {large }}}^{[[]}$the integrand probability is uniformly of order $O(\exp (-K \alpha))$. We now conclude our claim for $K$ large enough in view of the lower bound (8.65).

\subsection{Uniqueness of the large contour, excluding intermediate con- tours}

It follows by the previous Subsection 8.3 that, conditionally on the event $\left\{\mathbb{M}_{L}\left(\mathcal{A}^{[\beta]}\right)>\right.$ $\mathbb{M}[\beta] \pi L^{2}+a L^{2}, \quad \mathcal{N}[\alpha, L]$ holds $\}$, with overwhelming probability there exists one large contour $\theta_{\text {large }}$ of length $L \sqrt{\frac{2 \pi a}{|\mathbb{M}[\beta]|}}+O(\alpha)$, enclosing phase area $\frac{a L^{2}}{2|\mathbb{M}[\beta]|}+O(L \alpha)$, and this is the only $K \alpha$-large contour of $\mathcal{A}^{[\beta]}$ hitting $\mathbb{B}_{2}(L)$, with $K$ large enough. Below, we argue that for sufficiently large $C_{\text {large }}$, with overwhelming conditional probability, $\theta_{\text {large }}$ is in fact the unique $C_{\text {large }} \log L$-large contour of $\mathcal{A}^{[\beta]}$ hitting $\mathbb{B}_{2}(L)$. The first step in this direction is showing in Lemma 8.2, similar to Lemma 4.2 .4 in [12], that the phase of $K \alpha$-large contours adjusts very tightly to the micro-canonical constraint $\mathbb{M}_{L}\left(\mathcal{A}^{[\beta]}\right)>[\mathbb{M}[\beta] \pi+a] L^{2}$ 
and, roughly speaking, 'not much work is left for small contours'. Next, in Lemma 8.3 we use this knowledge to deduce the uniqueness of the large contour $\theta_{\text {large }}$ and to exclude the presence of any other $C_{\text {large }} \log L$-large contours with overwhelming probability under the micro-canonical constraint.

To proceed with the first of the afore-mentioned steps, we claim first that

Lemma 8.2 With $K$ as specified above we have

$$
\begin{aligned}
& \mathbb{P}\left([\mathbb{M}[\beta] \pi+a] L^{2}-\mathbb{E}\left(\mathbb{M}_{L}\left(\mathcal{A}^{[\beta]}\right) \mid \mathbb{L}_{K \alpha ; L}\right)>L^{4 / 3} \mid\right. \\
& \left.\mathbb{M}_{L}\left(\mathcal{A}^{[\beta]}\right)>[\mathbb{M}[\beta] \pi+a] L^{2}, \mathcal{N}[\alpha, L] \text { holds }\right)=o(1) .
\end{aligned}
$$

Proof We set

$$
\rho=\rho[L]:=L^{7 / 12}
$$

Applying Lemma 6.1 and Corollary 6.2 we get

$$
\begin{gathered}
\mathbb{P}\left([\mathbb{M}[\beta] \pi+a] L^{2}-\mathbb{E}\left(\mathbb{M}_{L}\left(\mathcal{A}^{[\beta]}\right) \mid \mathbb{L}_{K \alpha ; L}\right)>L^{4 / 3}, \mathbb{M}_{L}\left(\mathcal{A}^{[\beta]}\right)>[\mathbb{M}[\beta] \pi+a] L^{2}\right) \leq \\
\sum_{\Sigma} \mathbb{P}\left(\mathbb{M}_{L}\left(\mathcal{A}^{[\beta]}\right)>[\mathbb{M}[\beta] \pi+a] L^{2},[\mathbb{M}[\beta] \pi+a] L^{2}-\mathbb{E}\left(\mathbb{M}_{L}\left(\mathcal{A}^{[\beta]}\right) \mid \mathbb{L}_{K \alpha ; L}\right)>L^{4 / 3},\right. \\
\left.\mathbb{L}_{K \alpha ; L} \stackrel{(K \alpha, \delta)}{\propto} \Sigma\right)
\end{gathered}
$$

with the sum ranging over all $(K \alpha, \delta)$-skeletons $\Sigma$ contained in $\mathbb{B}_{2}(L)$ and with $\stackrel{(K \alpha, \delta)}{\propto}$ used as an indexed version of $\propto$ to denote the well-covering relation of $(K \alpha, \delta)$-contours by $(K \alpha, \delta)$-skeletons. Use the exponential tightness results of Lemma 2.1 in Section 2 to conclude that, with arbitrarily large $C_{1}$ and with $C_{2}$ large enough, this sum can be bounded above by

$$
\begin{gathered}
\sum_{\Sigma, \text { length }(\Sigma) \in\left[L \sqrt{\frac{2 \pi a}{|\mathbb{M}[\beta]|}}-\rho, C_{2} L\right]} \mathbb{P}\left([\mathbb{M}[\beta] \pi+a] L^{2}-\mathbb{E}\left(\mathbb{M}_{L}\left(\mathcal{A}^{[\beta]}\right) \mid \mathbb{L}_{K \alpha ; L}\right)>L^{4 / 3}, \mathbb{L}_{K \alpha ; L} \stackrel{(K \alpha, \delta)}{\propto} \Sigma\right) \\
+\sum_{\Sigma, \text { length }(\Sigma)<L \sqrt{\frac{2 \pi a}{\mid \mathbb{M}[\beta]}}-\rho} \mathbb{P}\left(\mathbb{M}_{L}\left(\mathcal{A}^{[\beta]}\right)>[\mathbb{M}[\beta] \pi+a] L^{2}, \mathbb{L}_{K \alpha ; L} \stackrel{(K \alpha, \delta)}{\propto} \Sigma\right)+\exp \left(-C_{1} L\right) .
\end{gathered}
$$

We proceed by showing that all consecutive terms in (8.75), for brevity denoted below by $P_{1}, P_{2}$ and $P_{3}$ respectively, are negligibly small compared to the probability $P_{4}:=$ 
$\mathbb{P}\left(\mathbb{M}_{L}\left(\mathcal{A}^{[\beta]}\right)>[\mathbb{M}[\beta] \pi+a] L^{2}, \mathcal{N}[\alpha, L]\right.$ holds $)$. To begin with the first term $P_{1}$, use Remark 8.1 to conclude that the number of summands in this sum is of order $\exp \left(O\left(\alpha^{-1} L \log L\right)\right)$. Moreover, applying Lemma 6.4, noting that conditionally on $\mathbb{L}_{K \alpha ; L}=\gamma$ the field $\mathcal{A}^{[\beta]}$ coincides in law with $\mathcal{A}_{\mathbb{R}^{2}: \gamma}^{[\beta] ; \alpha, \mathbb{B}_{2}(L)} \cup \gamma$ and using Theorem 3.1 conditionally on $\mathbb{L}_{K \alpha ; L}$, we uniformly bound above each summand of $P_{1}$ by

$$
\begin{gathered}
\exp \left(-\left[\sqrt{\frac{2 \pi a}{|\mathbb{M}[\beta]|}} L-\rho\right] \tau_{K \alpha}^{[\beta]}\right) \exp \left(-c\left[\frac{L^{8 / 3}}{L^{2}} \wedge \frac{L^{4 / 3}}{K \alpha}\right]\right)= \\
\exp \left(-\sqrt{\frac{2 \pi a}{|\mathbb{M}[\beta]|}} L \tau_{K \alpha}^{[\beta]}+O(\rho)\right) \exp \left(-c L^{2 / 3}\right) .
\end{gathered}
$$

Recalling the definition of $\alpha=\alpha[L]=\sqrt{L} \log L, \rho=L^{7 / 12}$ and using the lower bound (8.65) of Theorem 1.2 with $\alpha$ replaced there by $K \alpha$, we conclude that

$$
P_{1}=o\left(P_{4}\right) .
$$

To show that

$$
P_{2}=o\left(P_{4}\right)
$$

observe that, by isoperimetric Lemma $6.3, \operatorname{length}(\Sigma)<L \sqrt{\frac{2 \pi a}{|\mathbb{M}[\beta]|}}-\rho$ implies that Area $(\Sigma)<$ $\frac{a L^{2}}{2|\mathbb{M}[\beta]|}-\Omega(L \rho)$, whence, by $(3.20), \mathbb{E}\left(\mathbb{M}_{L}\left(\mathcal{A}^{[\beta]}\right) \mid \mathbb{L}_{K \alpha ; L}\right) \leq[\mathbb{M}[\beta] \pi+a] L^{2}-\Omega(L \rho)$ almost surely on the event $\left\{\mathbb{L}_{K \alpha ; L} \stackrel{(K \alpha, \delta)}{\propto} \Sigma\right\}$. Consequently, by Theorem 3.1 applied conditionally on $\mathbb{L}_{K \alpha ; L}$, each summand in $P_{2}$ is bounded above by $\exp \left(-c\left[\frac{L^{2} \rho^{2}}{L^{2}} \wedge \frac{L \rho}{\alpha}\right]\right)=\exp \left(-c \frac{L \rho}{\alpha}\right)$. Using the lower bound (8.65) of Theorem 1.2 (with $\alpha$ replaced there by $K \alpha$ ) and recalling that $\frac{\rho L}{\alpha} \gg L$ we obtain (8.77). Observing that, by the same lower bound (8.65), $P_{3}=o\left(P_{4}\right)$ provided $C_{1}$ is chosen large enough, we complete the proof of the Lemma by combining (8.76) and (8.77).

As announced above, our next statement will allow us to exclude with overwhelming conditional probability under the micro-canonical constraint the presence of $C_{\text {large }} \log L$ large contours different than $\theta_{\text {large }}$.

Lemma 8.3 There exists a constant $C_{\text {large }}>0$ such that uniformly in collections $\gamma$ of $K \alpha$-large contours in $\mathbb{B}_{2}(L)$ with length $(\gamma) \leq L \log L$ and in $\Delta \leq L^{4 / 3}$ we have

$$
\begin{gathered}
\mathbb{P}\left(\mathcal{A}_{\mathbb{R}^{2}: \gamma}^{[\beta] ; K \alpha, \mathbb{B}_{2}(L)} \text { contains a } C_{\text {large }} \log L \text {-large contour } \mid \mathbb{M}_{L}\left(\mathcal{A}_{\mathbb{R}^{2}: \gamma}^{[\beta] ; K \alpha, \mathbb{B}_{2}(L)} \cup \gamma\right)>\right. \\
\left.\mathbb{E} \mathbb{M}_{L}\left(\mathcal{A}_{\mathbb{R}^{2}: \gamma}^{[\beta] ; \alpha, \mathbb{B}_{2}(L)} \cup \gamma\right)+\Delta\right)=o(1) .
\end{gathered}
$$


Proof For brevity write

$$
\mu_{L, \gamma}^{K \alpha}:=\mathbb{M}_{L}\left(\mathcal{A}_{\mathbb{R}^{2}: \gamma}^{[\beta] ;, \mathbb{B}_{2}(L)} \cup \gamma\right), \mu_{L, \gamma}^{K \alpha, h}:=\mathbb{M}_{L}\left(\mathcal{A}_{\mathbb{B}_{2}(L): \gamma}^{[\beta, h] ;, \mathbb{B}_{2}(L)} \cup \gamma\right)
$$

and let $\mathcal{E}\left[C_{\text {large }}, L\right]$ be the event that $\mathcal{A}_{\mathbb{R}^{2}: \gamma}^{[\beta], \mathbb{B}_{2}(L)}$ contains no $C_{\text {large }} \log L$-large contours hitting $\mathbb{B}_{2}(L)$ and $\mathcal{E}^{h}\left[C_{\text {large }}, L\right]$ the event that $\mathcal{A}_{\mathbb{B}_{2}(L): \gamma}^{[\beta, h] K \alpha, \mathbb{B}_{2}(L)}$ contains no $C_{\text {large }} \log L$-large contours hitting $\mathbb{B}_{2}(L)$. From Corollary 3.5 it follows in particular that for each $\eta \in$ $\left[\Delta, L^{4 / 3} \log L\right]$ there exists a unique value of the external magnetic field $h[\eta, L]:=h[\eta, L, \gamma]=$ $\Theta\left(\eta / L^{2}\right)$ such that

$$
\mathbb{E} \mu_{L, \gamma}^{K \alpha, h[\eta, L]}=\mathbb{E} \mu_{L, \gamma}^{K \alpha}+\eta
$$

and, moreover, $h[\eta, L]$ increases with $\eta$ given $L$, whence $h[\eta, L] \in\left[h^{-}[L], h^{+}[L]\right]$ with

$$
h^{-}[L]:=h[\Delta, L]=\Theta\left(\Delta / L^{2}\right)
$$

and

$$
h^{+}[L]:=h\left[L^{4 / 3} \log L, L\right]=\Theta\left(L^{-2 / 3} \log L\right) .
$$

For each $L>0$ we split the interval $\left[\Delta, L^{4 / 3} \log L\right]$ into $\Theta\left(h^{+}[L] L^{4 / 3} \log L\right)=\Theta\left(L^{2 / 3} \log ^{2}(L)\right)$ equal-sized subintervals $\left[\Delta=\eta_{0}, \eta_{1}\right),\left[\eta_{1}, \eta_{2}\right), \ldots$ of length $\Theta\left(1 / h^{+}[L]\right)=\Theta\left(L^{2 / 3} / \log L\right)$ each and we put $h_{k, L}:=h\left[\frac{\eta_{k}+\eta_{k+1}}{2}, L\right]$. For each of the subintervals $\left[\eta_{k}, \eta_{k+1}\right)$ write

$$
\begin{aligned}
& \mathbb{P}\left(\mathcal{E}\left[C_{\text {large }}, L\right] \mid \mu_{L, \gamma}^{K \alpha} \in \mathbb{E} \mu_{L, \gamma}^{K \alpha}+\left[\eta_{k}, \eta_{k+1}\right)\right) \leq \\
& \frac{\exp \left(-h_{k, L}\left[\mathbb{E} \mu_{L, \gamma}^{K \alpha}+\eta_{k}\right]\right) \mathbb{P}\left(\mathcal{E}^{h_{k, L}}\left[C_{\text {large }}, L\right] \text { holds, } \mu_{L, \gamma}^{K \alpha, h_{k, L}} \in \mathbb{E} \mu_{L, \gamma}^{K \alpha}+\left[\eta_{k}, \eta_{k+1}\right)\right)}{\exp \left(-h_{k, L}\left[\mathbb{E} \mu_{L, \gamma}^{K \alpha}+\eta_{k+1}\right]\right) \mathbb{P}\left(\mu_{L, \gamma}^{K \alpha, h_{k, L}} \in \mathbb{E} \mu_{L, \gamma}^{K \alpha}+\left[\eta_{k}, \eta_{k+1}\right)\right)} \leq \\
& \exp \left(h_{k, L}\left[\eta_{k+1}-\eta_{k}\right]\right) \mathbb{P}\left(\mathcal{E}^{h_{k, L}}\left[C_{\text {large }}, L\right]|| \mu_{L, \gamma}^{K \alpha, h_{k, L}}-\mathbb{E} \mu_{L, \gamma}^{K \alpha, h_{k, L}} \mid \leq \frac{\eta_{k+1}-\eta_{k}}{2}\right) .
\end{aligned}
$$

At this point we claim that

$$
\mathbb{P}\left(\left|\mu_{L, \gamma}^{K \alpha, h_{k, L}}-\mathbb{E} \mu_{L, \gamma}^{K \alpha, h_{k, L}}\right| \leq \frac{\eta_{k+1}-\eta_{k}}{2}\right)=\Omega\left(L^{-1 / 3} \log ^{-1} L\right)
$$

uniformly in $L, \gamma, \Delta, k$. Note that this is in fact a rather weak statement in the spirit of local central limit theorem (LCLT) and an LCLT could in principle be established for the polygonal Markov fields in its full strength much along the same lines as Lemma 
2.4.1 in [12], with standard modifications due to the non-lattice nature of our setting. However, since we only need the weaker relation (8.80), we provide a much shorter argument specialised for this case. To this end, we subdivide the disk $\mathbb{B}_{2}(L)$ into $\Theta(L)$ equal-sized squares $Q_{1, L}, Q_{2, L}, \ldots$ of side length $\Theta(\sqrt{L})$, separated by moats of width $\log ^{2}(L)$. Now, in view of (1.10), the family of identically distributed random variables

$$
X_{i, L}:=\mathbb{M}_{Q_{i, L}}\left(\mathcal{A}_{\mathbb{B}_{2}(L): \gamma}^{\left[\beta, h_{k, L}\right] ; K \alpha, \mathbb{B}_{2}(L)} \cup \gamma\right),
$$

can be coupled with a sequence of i.i.d. copies $\hat{X}_{i, L}$ of $X_{i, L}$ in the way that $\mathbb{P}\left(\exists_{i} X_{i, L} \neq\right.$ $\left.\hat{X}_{i, L}\right)=O\left(L^{2} \exp \left(-c \log ^{2}(L)\right)\right), c>0$. Indeed, $O\left(L^{2} \exp \left(-c \log ^{2}(L)\right)\right)$ is the order of the probability that the ancestor clans arising for different $Q_{i, L}$ in the graphical construction of Subsubsection 1.2.2 are not all pairwise disjoint. Write

$$
Y_{L}:=\mathbb{M}_{\mathbb{B}_{2}(L) \backslash \bigcup_{i} Q_{i, L}}\left(\mathcal{A}_{\mathbb{B}_{2}(L): \gamma}^{\left[\beta, h_{k, L}\right] ; K \alpha, \mathbb{B}_{2}(L)} \cup \gamma\right)
$$

and note that

$$
\mu_{L, \gamma}^{h_{k, L}, K \alpha}=\sum_{i} X_{i, L}+Y_{L}
$$

Further, observe that, in complete analogy with Theorem 3.1,

$$
\mathbb{P}\left(\left|Y_{L}-\mathbb{E} Y_{L}\right|>\sqrt{L} \log ^{3}(L)\right) \leq \exp \left(-c\left[\frac{\left[\sqrt{L} \log ^{3}(L)\right]^{2}}{L \log ^{2}(L)} \wedge \frac{\sqrt{L} \log ^{3}(L)}{K \alpha}\right]\right)=\mathrm{e}^{-c \log ^{2}(L)}
$$

Using the coupling of $X_{i, L}$ and $\hat{X}_{i, L}$ as discussed above, taking into account (8.81) and (8.82) and recalling that $\eta_{k+1}-\eta_{k}=\Theta\left(L^{2 / 3} / \log L\right) \gg \sqrt{L} \log ^{3}(L)$ we can now deduce the required relation (8.80) by the classical local central limit theorem applied for $\sum_{i} \hat{X}_{i, L}$, use e.g. Theorem 1 in Wey [17] with $\lambda_{L}:=L^{2 / 3} / \log (L)$ and $M_{L}:=L^{2 / 3} / \log ^{2}(L)$ there, with the assumption (H1) [central limit theorem for $\left.\mu_{L, \gamma}^{K \alpha, h_{k, L}}\right]$ there following by Theorem 2.10.5 of [10] or Theorem 2.4.R5 and Section 5.3 in [9] with obvious modifications due to the continuum rather than lattice nature of our setting, and with the assumption (H2) in [17] satisfied in view of (3.25), (3.27) and by the relation $\operatorname{Var}\left[\mu_{L, \gamma}^{K \alpha, h_{k, L}}\right]=\Theta\left(L^{2}\right)$ which can be established along the same lines as (3.29).

Consequently, since $h_{k, L}\left[\eta_{k+1}-\eta_{k}\right]=O(1)$ in (8.79), combining the relation (8.79) with (8.80) and taking into account that $\mathbb{P}\left(\mathcal{E}^{h_{k, L}}\left[C_{\text {large }}, L\right]\right)=\exp \left(-\Omega\left(C_{\text {large }} \log L\right)\right)$ uniformly 
in $\Delta, L, k, \gamma$ for $C_{\text {large }}$ large enough in view of Lemma 2.1 in Section 2, we conclude that, uniformly in $\Delta, L, k, \gamma$

$$
\mathbb{P}\left(\mathcal{E}\left[C_{\text {large }}, L\right] \mid \mu_{L, \gamma}^{K \alpha} \in \mathbb{E} \mu_{L, \gamma}^{K \alpha}+\left[\eta_{k}, \eta_{k+1}\right)\right)=o(1)
$$

In view of (8.83) the assertion of our lemma will follow as soon as we show that

$$
\mathbb{P}\left(\mu_{L, \gamma}^{K \alpha}>\mathbb{E} \mu_{L, \gamma}^{K \alpha}+L^{4 / 3} \log L \mid \mu_{L, \gamma}^{K \alpha}>\mathbb{E} \mu_{L, \gamma}^{K \alpha}+\Delta\right)=o(1)
$$

uniformly in $\Delta, L, \gamma$. To this end, use Theorem 3.1 to conclude that

$$
\mathbb{P}\left(\mu_{L, \gamma}^{K \alpha}>\mathbb{E} \mu_{L, \gamma}^{K \alpha}+L^{4 / 3} \log L\right) \leq \exp \left(-c L^{2 / 3} \log ^{2}(L)\right)
$$

Next, apply Lemma 3.6 to get

$$
\mathbb{P}\left(\mu_{L, \gamma}^{K \alpha}>\mathbb{E} \mu_{L, \gamma}^{K \alpha}+\Delta\right) \geq \exp \left(-O\left([\Delta+L \log L]^{2} / L^{2}\right)\right) \geq \exp \left(-O\left(L^{2 / 3}\right)\right) .
$$

which yields the required relation (8.84) when combined with (8.85). The proof of the lemma is hence complete.

Recalling that conditionally on $\mathbb{L}_{K \alpha ; L}=\gamma$ the field $\mathcal{A}^{[\beta]}$ coincides in distribution with $\mathcal{A}_{\mathbb{R}^{2}: \gamma}^{[\beta] K \alpha, \mathbb{B}_{2}(L)} \cup \gamma$ and that, by the discussion in Subsection $8.3, \mathbb{L}_{K \alpha, L}=\left\{\theta_{\text {large }}\right\}$ with overwhelming probability under the micro-canonical constraint, and then combining Lemma 8.2 with Lemma 8.3 applied conditionally on $\gamma=\mathbb{L}_{K \alpha, L}$, shows that, conditionally on the event $\left\{\mathbb{M}_{L}\left(\mathcal{A}^{[\beta]}\right)>\mathbb{M}[\beta] \pi L^{2}+a L^{2}, \mathcal{N}[\alpha, L]\right.$ holds $\}$, with overwhelming probability $\theta_{\text {large }}$ is the only $C_{\max } \log L$-large contour of $\mathcal{A}^{[\beta]}$ hitting $\mathbb{B}_{2}(L)$. This completes the present subsection of the proof.

\subsection{Localising the large contour}

It remains to show that the large contour $\theta_{\text {large }}$ satisfies

$$
\min _{x} \rho_{H}\left(\theta_{\text {large }}, \mathbb{S}_{1}\left(x, L \sqrt{\frac{a}{2 \pi|\mathbb{M}[\beta]|}}\right)\right)=O\left(L^{3 / 4} \sqrt{\log L}\right)
$$

But this follows immediately by specialising to our setting for $\theta_{\text {large }}$ the inequality (2.4.1) in Section 2.4 of Dobrushin, Kotecký \& Shlosman [7] and combining it with (8.73) and (8.74). This completes the proof of Theorem 1.2. 


\section{Appendix}

Below, we discuss the dynamic representation and some further properties of the basic Arak process, see Arak \& Surgailis [2], Section 4 for the dynamic representation. For a fixed bounded open convex domain $D$ we shall construct the basic Arak process $\mathcal{A}_{D}^{*}$ with free boundary conditions (unlike in (1.1) where empty boundary conditions are imposed).

\subsection{Dynamic construction of the basic Arak process}

We interpret the domain $D$ as a set of time-space points $(t, y) \in D$, with $t$ referred to as the time coordinate and with $y$ standing for the spatial coordinate of a particle at the time $t$. In this language, a straight line segment in $D$ stands for a piece of the time-space trajectory of a freely moving particle. For a straight line $l$ non-parallel to the spatial axis and crossing the domain $D$ we define in the obvious way its entry point to $D, \operatorname{in}(l, D) \in \partial D$ and its exit point $\operatorname{out}(l, D) \in \partial D$.

We choose the time-space birth coordinates for the new particles according to a homogeneous Poisson point process of intensity $\pi$ in $D$ (interior birth sites) superposed with a Poisson point process on the boundary (boundary birth sites) with the intensity measure

$$
\kappa(B)=\mathbb{E} \operatorname{card}\{l \in \Lambda, \operatorname{in}(l, D) \in B\}, B \subseteq \partial D
$$

Each interior birth site emits two particles, moving with initial velocities $v^{\prime}$ and $v^{\prime \prime}$ chosen according to the joint distribution

$$
\theta\left(d v^{\prime}, d v^{\prime \prime}\right):=\pi^{-1}\left|v^{\prime}-v^{\prime \prime}\right|\left(1+v^{2}\right)^{-3 / 2}\left(1+v^{\prime \prime 2}\right)^{-3 / 2} d v^{\prime} d v^{\prime \prime}
$$

This can be shown to be equivalent to choosing the directions of the straight lines representing the space-time trajectories of the emitted particles according to the distribution of the typical angle between two lines of $\Lambda$, see Sections 3 and 4 in [2] and the references therein. It is also easily seen that the value of angle $\phi \in(0, \pi)$ between these lines is distributed according to the density $\sin (\phi) / 2$. Each boundary birth site $x \in \partial D$ yields one particle with initial speed $v$ determined according to the distribution $\theta_{x}(d v)$ identified by requiring that the direction of the line entering $D$ at $x$ and representing the time-space trajectory of the emitted particle be chosen according to the distribution of a straight line $l \in \Lambda$ conditioned on the event $\{x=\operatorname{in}(l, D)\}$.

All the particles evolve independently in time according to the following rules. 
(E1) Between the critical moments listed below each particle moves freely with constant velocity so that $d y=v d t$,

(E2) When a particle touches the boundary $\partial D$, it dies,

(E3) In case of a collision of two particles (equal spatial coordinates $y$ at some moment $t$ with $(t, y) \in D)$, both of them die,

(E4) The time evolution of the velocity $v_{t}$ of an individual particle is given by a pure-jump Markov process so that

$$
\mathbb{P}\left(v_{t+d t} \in d u \mid v_{t}=v\right)=q(v, d u) d t
$$

for the transition kernel

$$
q(v, d u):=|u-v|\left(1+u^{2}\right)^{-3 / 2} d u d t
$$

It is worth noting that, in full analogy with the discussion following (9.87), the (sharp) angle between the straight lines representing the space-time trajectories of the particle before and after the velocity update is distributed according to the typical angle between two lines of $\Lambda$.

It has been proven (see Lemma 4.1 in [2]) that with the above construction of the interacting particle system, the time-space trajectories traced by the evolving particles coincide in distribution with the Arak process $\mathcal{A}_{D}^{*}$ defined as in (1.1) with the family $\Gamma_{D}$ of admissible polygonal configurations extended to $\Gamma_{D}^{*}$ allowing also for partial contours chopped off by the boundary, which amounts to admitting not only internal vertices of degree 2, as in (P2), but also boundary vertices of degree 1.

\subsection{Properties of the basic Arak process}

As already mentioned in the introductory section, and as shown in Arak \& Surgailis [2], the basic Arak process $\mathcal{A}_{D}^{*}$ enjoys a number of striking properties. The two-dimensional germ Markov property, stating that the conditional distribution of the field inside a bounded region with piecewise smooth boundary given the outside configuration only depends on the trace of this configuration on the boundary (intersection points and intersection directions) is an immediate consequence of the Gibbsian definition. Next important property is the consistency: for bounded open and convex $D_{1}$ and $D_{2}$ with $D_{1} \subseteq D_{2}$ the restriction of $\mathcal{A}_{D_{2}}^{*}$ 
to $D_{1}$ coincides in distribution with $\mathcal{A}_{D_{1}}^{*}$, see Theorem 4.1 ibidem. This immediately allows us to define the infinite volume Arak process $\mathcal{A}$, which inherits the isometry invariance of the finite volume Gibbsian definition and which is a thermodynamic limit for $\mathcal{A}^{[0]}$. By the results of Schreiber [14], this corresponds to the unique infinite-volume bounded-density stationary evolution of the particle system discussed in Subsection 9.1 above. Interestingly, the intersection of the Arak process $\mathcal{A}$ with any fixed straight line is a Poisson point process of intensity 2, see [2], which gives us direct access to two-point correlation functions of $\mathcal{A}$ under the colouring as in Subsection 1.1. Moreover, the partition function for the Arak process can be explicitly evaluated: it is known that

$$
\mathbb{E} \sum_{\delta \in \Gamma_{D}^{*}\left(\Lambda_{D}\right)} \exp (-2 \text { length }(\delta))=\exp (\pi \operatorname{Area}(D)),
$$

see Theorem 4.1 in [2] [note that the prefactor $2 \exp (\operatorname{length}(\partial D))$, present in the quoted theorem, is absent here because we take the law of $\Lambda$ rather than the unnormalised measure $\mu^{*}$ as the reference measure and, moreover, we do not sum over two different admissible black/white colourings of each polygonal configuration]. It should be emphasised that these exact results are only available for $\mathcal{A}$ and not for $\mathcal{A}^{[\beta]}, \beta>0$.

Interestingly, there exists a much broader class of consistent polygonal Markov fields admitting analogous dynamic representations, possibly enhanced to allow for vertices of higher degrees (3 and 4), see ibidem. The question of characterising the class of all polygonal Markov fields admitting dynamic representations is far from being trivial and falls beyond the scope of this article. A conjectured description of this class has been provided in Arak, Clifford \& Surgailis [4], where a very nice alternative point- rather than line-based representation of polygonal fields is also discussed.

Acknowledgements The author gratefully acknowledges the support of the Foundation for Polish Science (FNP), the Polish Minister of Scientific Research and Information Technology grant 1 P03A 01828 (2005-2007) and from the EC 6th Framework Programme Priority 2 Information Society Technology Network of Excellence MUSCLE (Multimedia Understanding through Semantics, Computation and Learning; FP6-507752). He also wishes to express his gratitude for hospitality of the Centrum voor Wiskunde en Informatica (CWI), Amsterdam, The Netherlands, where a part of this research was completed. Special thanks are due to anonymous referees whose remarks have been helpful in improving this paper. 


\section{References}

[1] Arak, T. (1982) On Markovian random fields with finite number of values, 4th USSR-Japan symposium on probability theory and mathematical statistics, Abstracts of Communications, Tbilisi.

[2] Arak, T., Surgailis, D. (1989) Markov Fields with Polygonal Realisations, Probab. Th. Rel. Fields 80, 543-579.

[3] Arak, T., Surgailis, D. (1991) Consistent polygonal fields, Probab. Th. Rel. Fields 89, 319-346.

[4] Arak, T., Clifford, P., Surgailis, D. (1993) Point-based polygonal models for random graphs, Adv. Appl. Probab. 25, 348-372.

[5] Baryshnikov, Yu., Yukich, J.E. (2005) Gaussian Limits for Random Measures in Geometric Probability, Annals of Appl. Prob. 15, 213-253.

[6] Bodineau, T., Ioffe, D., Velenik, I. (2000) Rigorous probabilistic analysis of equilibrium crystal shapes, Journal of Statistical Physics, 41, 1033-1098.

[7] Dobrushin, R., Kotecký, R., Shlosman, S. (1992) Wulff construction: a global shape from local interaction, AMS translation series, Providence RI, 104.

[8] Dobrushin, R., Shlosman, S. (1994) Large and moderate deviations in the Ising model. Adv. in Soviet Math. 20, 91-220.

[9] Fernández, R., Ferrari, P., Garcia, N. (1998) Measures on contour, polymer or animal models. A probabilistic approach. Markov Processes and Related Fields 4, 479-497.

[10] Fernández, R., Ferrari, P., Garcia, N. (2001) Loss network representation of Ising contours Ann. Probab. 29, 902-937.

[11] Fernández, R., Ferrari, P., Garcia, N. (2002) Perfect simulation for interacting point processes, loss networks and Ising models. Stoch. Proc. Appl. 102, 63-88.

[12] Ioffe, D., Schonmann, R. (1998) Dobrushin-Kotecký-Shlosman theory up to the critical temperature, Comm. Math. Phys. 199, 117-167. 
[13] Nicholls, G.K. (2001) Spontaneous magnetisation in the plane, Journal of Statistical Physics, 102, 1229-1251.

[14] Schreiber, T. (2004a) Mixing properties for polygonal Markov fields in the plane, submitted, available at: http://www.mat.uni.torun.pl/preprints,18-2003.

[15] Schreiber, T. (2005) Random dynamics and thermodynamic limits for polygonal Markov fields in the plane, Advances in Applied Probability 37, 884-907.

[16] Surgailis, D. (1991) Thermodynamic limit of polygonal models, Acta applicandae mathematicae, 22, 77-102.

[17] Wey, S. (1995) Un théorème limite local, C. R. Acad. Sci. Paris; Sér. I, 320, 997-1002. 